\title{
TILTED FIBER GRATING REFRACTIVE INDEX SENSOR AND INTERROGATION DEVICE
}

\author{
BY \\ Amir Jafari \\ A THESIS SUBMITTED TO THE FACULTY OF GRADUATE STUDIES AND RESEARCH \\ IN PARTIAL FULFILLMENT OF THE REQUIREMENTS FOR THE DEGREE OF \\ MASTER OF SCIENCE \\ OTTAWA-CARLETON INSTITUTE FOR ELECTRICAL AND COMPUTER ENGINEERING \\ FACULTY OF ENGINEERING \\ DEPARTMENT OF ELECTRONICS \\ CARLETON UNIVERSITY \\ OTTAWA, ONTARIO \\ MAY $30^{\mathrm{TH}}, 2006$
}

(C) Copyright by Amir Jafari, 2006 


$\begin{array}{ll}\begin{array}{l}\text { Library and } \\ \text { Archives Canada }\end{array} & \begin{array}{l}\text { Bibliothèque et } \\ \text { Archives Canada }\end{array} \\ \begin{array}{l}\text { Published Heritage } \\ \text { Branch }\end{array} & \begin{array}{l}\text { Direction du } \\ \text { Patrimoine de l'édition }\end{array} \\ \begin{array}{l}\text { 395 Wellington Street } \\ \text { Ottawa ON K1A ON4 }\end{array} & \begin{array}{l}\text { 395, rue Wellington } \\ \text { Ottawa ON K1A ON4 } \\ \text { Canada }\end{array}\end{array}$

Your file Votre référence ISBN: 978-0-494-18316-8 Our file Notre référence ISBN: 978-0-494-18316-8

NOTICE:

The author has granted a nonexclusive license allowing Library and Archives Canada to reproduce, publish, archive, preserve, conserve, communicate to the public by telecommunication or on the Internet, loan, distribute and sell theses worldwide, for commercial or noncommercial purposes, in microform, paper, electronic and/or any other formats.

The author retains copyright ownership and moral rights in this thesis. Neither the thesis nor substantial extracts from it may be printed or otherwise reproduced without the author's permission.
AVIS:

L'auteur a accordé une licence non exclusive permettant à la Bibliothèque et Archives Canada de reproduire, publier, archiver, sauvegarder, conserver, transmettre au public par télécommunication ou par l'Internet, prêter, distribuer et vendre des thèses partout dans le monde, à des fins commerciales ou autres, sur support microforme, papier, électronique et/ou autres formats.

L'auteur conserve la propriété du droit d'auteur et des droits moraux qui protège cette thèse. $\mathrm{Ni}$ la thèse ni des extraits substantiels de celle-ci ne doivent être imprimés ou autrement reproduits sans son autorisation.
In compliance with the Canadian

Privacy Act some supporting forms may have been removed from this thesis.

While these forms may be included in the document page count, their removal does not represent any loss of content from the thesis.
Conformément à la loi canadienne sur la protection de la vie privée, quelques formulaires secondaires ont été enlevés de cette thèse.

Bien que ces formulaires aient inclus dans la pagination, il n'y aura aucun contenu manquant.

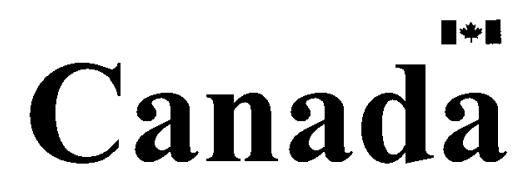




\section{Abstract}

This masters thesis presents a new way to measure the refractive index using Tilted Fiber Bragg Grating. We start by studying the properties of the TFBG spectrum in different surrounding refractive indices. We show that the notches of the TFBG spectrum displace as the surrounding refractive index changes. By picking up a particular notch (i.e. a particular mode) and tracking the shifts in the position of the center wavelength of this mode, we could generate curves by use of which one can determine the refractive index of the surrounding material. This property of the TFBG spectrum based on which the position of the modes displaces in response to the change in the surrounding refractive index is due to the fact that the tilt angle of the grating planes makes the light to couple to the cladding modes and because these cladding modes travel close to the cladding-external material surface, they can sense the change in the external material refractive index. Another interesting point mentioned in this research was that one does not need to worry about the shape of the mode used to measure the refractive index. This is true because as long as the modes have the same distance from Bragg resonance, they react exactly the same to the change in the external refractive index.

The second part of the thesis dealt with the Arrayed Waveguide Grating as a promising device to be used as the interrogator of the TFBG refractive index sensor. A very preliminary study was performed on the effect of fabrication errors on the performance of AWG. An optimum design based on the requirements of an ideal interrogator unit, in terms of crosstalk, channel spacing and device size was presented. This design was simulated and the results of the simulation were presented. 


\section{Acknowledgements}

I would like to thank Dr. Jacques Albert, my thesis supervisor, for his continuous support and encouragement throughout my Masters program. He was always available for fruitful discussions, and his ideas and editorial efforts have greatly improved the quality of this dissertation. Thank you sir for every single word I learnt from you.

I am thankful to Dr. George Xiao of the National Research Council of Canada for his great help and scientific support during my stay in NRC and his acting as an examining committee member. I should also thank Dr. JianPing Yao of the University of Ottawa, Dr. Alan Steele and Dr. Barry Syrett of the Department of Electronics, for their willingness to act as examining committee members, and for their helpful comments and advice.

I would also like to thank all my friends in our Department, especially my colleagues Lingyun Xiong, Chengkun Chen, Alexander Andreyuk and Albane Laronche. I have to thank Adam BP. Froimovitch who helped me a lot by reading the thesis and editing it. I should thank my friend Pirouz Zarrinkhat for his lending me his thesis template. I should express my special thanks to the following individuals (and they know why): Johann S. Bach, Wolfgang A. Mozart, Ludwig van Beethoven and Hafez.

Last, but not least, I am truly grateful to my family, for their love and support.

Ottawa, May 2, 2006

Amir Jafari 
To Mitra, the SUN-GOD,

Who has been the source of life and love for me. 


\section{Table of Contents}

Table of Contents $\quad$ v

List of Tables vii

List of Figures viii

List of Notations and Abbreviations xii

1 Introduction $\quad 1$

1.1 Fiber Bragg Grating Based Sensors . . . . . . . . . . . . . . . . . . 1

1.2 AWG based Interrogators . . . . . . . . . . . . . . . . . . 3

1.3 Organization of the Thesis . . . . . . . . . . . . . . 4

2 Arrayed Waveguide Grating 6

2.1 AWG as a sensor interrogator ................... 6

2.2 Operating Principles . . . . . . . . . . . . . . . . . . . . 7

2.3 Grating Equation . . . . . . . . . . . . . . . . . . . . . 9

2.4 System Specifications of AWG . . . . . . . . . . . . . . 10

2.4.1 Channel Spacing ..................... 11

2.4.2 Channel Bandwidth . . . . . . . . . . . . . . . 11

2.4.3 Channel Crosstalk . . . . . . . . . . . . . . . . . 13

2.4 .4 Free Spectral Range . . . . . . . . . . . . . . . . . . . . . 16

2.4 .5 Loss . . . . . . . . . . . . . . . . . . 16

2.5 Design Procedure . . . . . . . . . . . . . . . . . . . . . . . . . . . . . . . . . . . . .

2.6 Fabrication tolerance . . . . . . . . . . . . . . 23

2.7 Interrogation . . . . . . . . . . . . . . . . 26

2.8 Conclusion . . . . . . . . . . . . . . . . . 29 
3 Tilted Fiber Bragg Grating: Concepts and Theory 30

3.1 Simple Bragg grating . . . . . . . . . . . . . . . . . 30

3.1.1 Strain and Temperature Sensitivity of FBG . . . . . . . 35

3.2 Tilted Fiber Bragg Grating . . . . . . . . . . . . . . . . 36

3.2.1 Cladding Mode Coupling in TFBGs . . . . . . . . . . . . . 37

3.3 Fabrication . . . . . . . . . . . . . . . 40

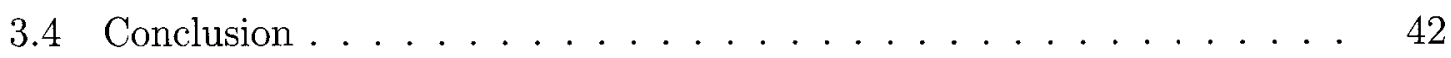

4 AWG as Sensor Interrogator, Simulation and Results 44

4.1 Requirements . . . . . . . . . . . . . . . . 44

4.2 Design and Simulation . . . . . . . . . . . . . . . . . . 45

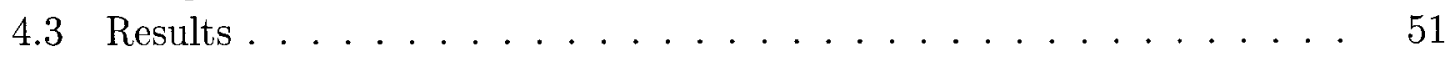

4.4 Conclusion . . . . . . . . . . . . . . . 56

5 TFBG as a Refractive Index Sensor, Experiment, Simulation and Results $\quad \mathbf{5 7}$

5.1 Concept Review . . . . . . . . . . . . . . . 57

5.2 Experiment Setup . . . . . . . . . . . . . . . . . 58

5.3 Simulation . . . . . . . . . . . . . . . . . . . . . . . . . . . . 65

5.4 Interrogation of the TFBG with $\mathrm{AWG} \ldots \ldots . \ldots 70$

5.5 Conclusion . . . . . . . . . . . . . . . 73

6 Concluding Remarks $\quad \mathbf{7 5}$

6.1 Summary of Thesis Research . . . . . . . . . . . . 75

6.2 Possible Future Work . . . . . . . . . . . . . . . 77

$\begin{array}{lll}\text { A AWG Based Interrogator Design Paramaters } & 78\end{array}$

$\begin{array}{lr}\text { Bibliography } & 81\end{array}$ 


\section{List of Tables}

2.1 System Specifications of an AWG(detailed descriptions follow). . . . . 11

2.2 Geometric Parameters of a $A W G$; $i$ stands for input, o stands for output and a stands for array. . . . . . . . . . . . . . . . . 12

2.3 Crosstalk Sources in an $A W G \ldots \ldots$. . . . . . . . . . . 16

2.4 Loss Mechanisms in an $A W G \ldots \ldots$

4.1 Comparison between 3 different $A W G$ designs . . . . . . . . . 50

A.1 Optical Parameters of Design 3 . . . . . . . . . . . . . 79

A.2 Mask Parameters of Design 3 . . . . . . . . . . . . . 79

A.3 System Specifications of Design 3 . . . . . . . . . . . 80 


\section{List of Figures}

2.1 A schematic layout of an AWG, Different parts of the device such as input waveguides, arrayed waveguides, free propagation region and output waveguides are marked on the picture. . . . . . . . . . . . 8

2.2 Deviating from the operating wavelength $\lambda_{c}$, tilts the wave front at the output aperture and therefore the focal point will shift along the image plane. ........................... 10

2.3 AWG output response. . . . . . . . . . . . . . . . 22

2.4 Fabrication imperfections: core size inaccuracy . . . . . . . . . . . 24

2.5 Fabrication imperfections: refractive index deviation . . . . . . . . 25

3.1 Illustration of a uniform Bragg grating. Also shown are incident, reflected and grating wavevectors. . . . . . . . . . . . . . 32

3.2 Reflection spectrum of a Bragg grating as a function of wavelength

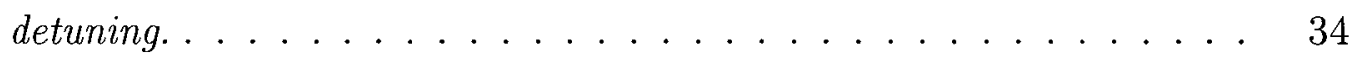

3.3 Illustration of a Tilted fiber Bragg Grating. Also shown are incident, reflected and grating wavevectors. . . . . . . . . . . . 37

3.4 A Typical TFBG Transmission Spectrum . . . . . . . . . . . 38

viii 
3.5 a: Overlap of the transmission spectra of a TFBG with two different external refractive indices, $b$ : The Bragg resonances overlap in two cases because the temperature is fixed and the core mode is not sensitive to the external refractive index change, $c$ : The cladding modes are sensitive to the external refractive index change. the solid line represents the spectrum when $n_{\text {ext }}=1.4$ and the dashed line corresponds to the case of $n_{\text {ext }}=1.42 \ldots \ldots \ldots \ldots$

3.6 Phase-mask technique setup to write a FBG (from "Fiber Bragg Grating Technology, Fundamentals and Overview" By K. O. Hill and G. Meltz, J. Lightwave Technol. vol 15, no 8, pp 1263-1276, August 1997.)

3.7 Phase-mask technique setup to write a $T F B G$. . . . . . . . . .

4.1 Experimental transmission spectrum of a TFBG with a tilt angle of 6

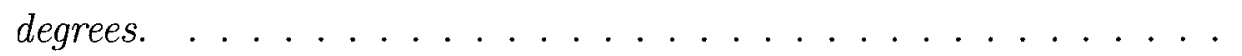

4.2 Spectrum of a TFBG with a tilt angle of 6 degrees, in the vicinity of the Bragg resonance. . . . . . . . . . . . . . . . 46

4.3 Spectrum of a TFBG with a tilt angle of 6 degrees, far from the Bragg resonance ......................... 46

4.4 Cross section of the waveguide used in this work . . . . . . . . . . . 48

4.5 Transmission Spectrum of the $A W G \ldots \ldots$. . . . . . . . . 49

4.6 Transmission Spectrum of design 1 . . . . . . . . . . . 50

4.7 Transmission Spectrum of design 2 ................. 51

4.8 Transmission Spectrum of the design 3 . . . . . . . . . . 52

4.9 Relationship between crosstalk and output waveguide spacing . . . . . 53

4.10 Type of taper used in output coupler section . . . . . . . . . . . . 53

4.11 Type of taper used in arrayed waveguides section . . . . . . . . . . 54

4.12 Transmission Spectrum of the design 3 with tapers . . . . . . . . . . 55

4.13 Relationship between bandwidth and output waveguide spacing . . . . 56 
5.1 Overlap of the spectrums of a TFBG in the air (solid line) and in a material with a refractive index different from air (dashed line). . . . 58

5.2 Schematic diagram of the experiment Setup) . . . . . . . . . . . 59

5.3 Abbe Refractometer, Principles of Operation. . . . . . . . . . . . 60

5.4 A Sample transmission spectrum of TFBG in sugar solution $\left(n_{D}=1.401\right) .61$

5.5 Transmission spectrum of TFBG in different SRIs in the vicinity of Bragg resonance. .................... . . 62

5.6 Transmission spectrum of TFBG in different SRIs from $1520 \mathrm{~nm}$ to $1530 n m \ldots \ldots \ldots 63$

5.7 Transmission spectrum of TFBG in different SRIs from $1535 \mathrm{~nm}$ to $1540 n m \ldots \ldots \ldots 63$

5.8 Shift in the Mode $L P_{(1,24)}$ due to the SRI change . . . . . . . . . 65

5.9 Sensitivity of Mode $L P_{(1,24)}$, (Unit of $\left.R I / p m\right)$. . . . . . . . . . . 66

5.10 Mode $L P_{(1,24)}$ shift due to SRI change, simulation and experiment. . . 67

5.11 Overlap of simulation and experimental curve using a linear mapping function to map RI of sugar-water solution from $589.3 \mathrm{~nm}$ to $1535 \mathrm{~nm}$. Error bars are also shown on the graph. The error in reading the RI value from Abbe Refractometer is $10^{-4}$ and the error in reading the wavelength is $\pm 3 p m . \ldots \ldots . \ldots . \ldots 69$

5.12 Shift in the location of modes $L P_{(1,24)}$ and $L P_{(3,23)}$ due to SRI change. 70

5.13 Power integrated over the output channel of an $A W G$ with the TFBG spectrum as the input. (a): Output of the AWG with the TFBG Spectrum in Air. (b): Output of the AWG with the TFBG Spectrum in $S R I=1.42\left(n_{D}=1.42\right)$. (c) : Output of the AWG with the TFBG Spectrum in $S R I=1.44\left(n_{D}=1.44\right) . \ldots \ldots \ldots$

5.14 Difference between the output responses of the $A W G$ with the output response of Air as the reference and, (a) : the output response of the $S R I=1.42$ subtracted from it; (b) : the output response of the $S R I=1.44$ subtracted from it. . . . . . . . . . . . . . 
5.15 Introducing discretization levels and converting the data to digital format. . . . . . . . . . . . . . . . 73 


\section{List of Notations and Abbreviations}

\begin{tabular}{c|l|c}
\hline Notation & Description & First Use \\
\hline FBG & Fiber Bragg Grating & 2 \\
RI & Refractive Index & 2 \\
$\lambda_{B}$ & Bragg Resonance & 2 \\
$n_{e f f}$ & Core effective refractive index & 2 \\
$\Lambda$ & RI modulation period & 2 \\
SRI & Surrounding Refractive Index & 3 \\
AWG & Arrayed Waveguide Grating & 3 \\
$N_{s}$ & Slab effective index & 7 \\
$\Delta \mathrm{L}$ & Length increment & 7 \\
$\mathrm{~m}$ & Grating order & 7 \\
$\Theta$ & Tilt angle & 7 \\
$N_{g}$ & Effective Index of Channel Waveguide & 7 \\
$\widetilde{N_{g}}$ & Group index/ $N_{g}$ & 7 \\
$N_{g r}$ & Group index & 8 \\
$\Delta \nu_{c}$ & Channel spacing & 9 \\
$\Delta \nu_{b w}$ & Channel bandwidth & 9 \\
$\chi_{t}$ & Crosstalk & 9 \\
$\Delta \nu_{F S R}$ & Free spectral range & 9 \\
$L_{n u}$ & Loss Non-uniformity & 9 \\
$L_{I}$ & Insertion loss & \\
\hline \hline
\end{tabular}

xii 


\begin{tabular}{c|l|c}
\hline Notation & Description & First Use \\
\hline$L_{D}$ & Diffraction loss & 9 \\
PD & Polarization dependence & 9 \\
BS & Bandpass shape & 9 \\
$\Delta \lambda$ & Channel spacing & 9 \\
$d_{o}$ & Output waveguide spacing & 9 \\
$d_{a}$ & Array waveguide spacing & 9 \\
$N$ & Number of waveguides in array & 10 \\
$N_{o}$ & Number of output waveguides & 10 \\
$L_{s}$ & Slab length & 10 \\
$G_{x}$ & Gap between waveguides (x:i,o and a for input, output and array) & 10 \\
$\lambda_{c}$ & Center wavelength & 14 \\
$n_{c o r e}$ & Refractive index of the core & 15 \\
$n_{c l a d}$ & Refractive index of cladding & 15 \\
$w_{w g}$ & Waveguide width & 15 \\
$N_{c h}$ & Number of channels & 18 \\
TFBG & Tilted Fiber Bragg Grating & 33 \\
$\lambda_{\text {clad,i }}$ & Resonance wavelength of a cladding mode & 36 \\
$\Lambda_{g}$ & $\Lambda$ cos $\left(\theta_{t}\right)$ & 36 \\
$\theta_{t}$ & Tilt angle relative to perpendicular fiber axis & 36 \\
$n_{D}$ & Refractive index at $589.3 \mathrm{~nm}$ & 58 \\
\hline \hline & & 9 \\
\hline
\end{tabular}


"Pauca sed matura. "1

- Gauß

\section{Chapter 1}

\section{Introduction}

"Ah, my Beloved, Fill the Cup that Clears

To-day of past Regrets and Future Fears

To - morrow? - Why, To - morrow I may be

Myself with Yesterday's Seven Thousand Years"2

$* * * * * * * * *$

\subsection{Fiber Bragg Grating Based Sensors}

Perceiving the surrounding world through sensing is the most exciting adventure of a newborn baby's life. The adventure that has led to the most dazzling scientific discoveries through centuries. In the modern world, where machines are being employed to make our lives easier, sensing the surrounding environment has taken on new dimensions. We need to sense temperature, pressure, strain, flow, load and tens

\footnotetext{
1 "Few, but ripe."

${ }^{2}$ Rubaiiat Khayyam Neyshabouri
} 
of other parameters in order to maintain the machines or structures which serve us. These new dimensions have made scientists and researchers put a lot of effort in to developing sensing technology. The research embraces sensing in its all entirety, from the principles of measurement to novel sensor technologies, deployment in the field and data analysis.

Of the novel technologies developed, one can name Fiber Optic Sensors or in particular Fiber Bragg Grating Sensors (FBG), the realization of which was due to the significant discovery of photosensitivity in silica fibers by Hill et. al. at the Communications Research Center in Canada. These fiber sensors show important features such as electrically passive operation, EMI immunity, high sensitivity and multiplexing capability. Add to these features the ease and low cost of fabrication, achieved by employing Ultraviolet (UV) inscribing technology and using proper phase mask to realize why these sensors are increasingly being used in real life. For a conventional grating, the periodicity of the index modulation is one half of the wavelength of the light meant to be reflected back.

$$
\lambda_{B}=2 n_{e f f} \Lambda
$$

where $\lambda_{B}$ is Bragg wavelength, $n_{e f f}$ is the effective refractive index and $\Lambda$ is periodicity of the index modulation. The coherent back reflection is the result of phase matching between the incident light and grating planes. Reflectivities of $100 \%$ and bandwidths between $0.1 \mathrm{~nm}$ and $60 \mathrm{~nm}$ are achievable. The flexible characteristics of FBG make them suitable for all kind of sensor applications. As the basic principle of the FBG sensors is based on the change of the reflected wavelength of the FBGs in response to strain and temperature, FBG sensors offer the advantage of self referencing, that is, the information is encoded as wavelength shift in these sensors which is an absolute quantity and does not suffer from the losses in the system (for intensity based sensors). 
FBG in their common form are not capable of sensing surrounding material refractive index (SRI) because the light is confined in the core and the propagation constant of the fundamental core mode is not affected by any SRI change. Introducing a tilt angle in the grating planes of a FBG will cause the light to scatter to cladding modes whose propagation constant is sensitive to the SRI. In this case, any change in the SRI leads to variation of the propagating constant of the mode and thus mode's resonance location displaces. This property can be used to construct a RI sensor.

\subsection{AWG based Interrogators}

In a sensing organism, if sensors are considered as the limbs that provide the information from surrounding environment, interrogator units should be considered as the limbs that perceive the information coming from sensors. During previous decades, there have been several interrogating technologies and schemes proposed to interrogate optical sensors (FBG based sensors) by people in the field (see Chapter 2). Many of the so-far proposed interrogating techniques of FBG based sensors exhibit several drawbacks. Some of them suffer from large size of interrogating unit while the others suffer from small wavelength range of operation, low accuracy, high fabrication and utilization cost and so on.

After introducing AWG as a multiplexer/demultiplexer device in the year of 1996 by Smit et. al. ([1]), there has been a huge attention attracted to this device. Most of this attention had come from people in telecommunications where they found some multiplexer/demultiplexer or router applications for this device. Application of AWG were not confined to telecommunication and finally this device found its way to sensor applications. In sensor applications, an AWG is considered as a device which is capable of fulfilling all the requirements of an ideal interrogator. It is compact in 
size and its fabrication can be at a low cost. By using $\mathrm{Si} / \mathrm{Si} \mathrm{O}_{2}$ fabrication technology, large scale fabrication is possible and thus commercial availability is guaranteed. By design, AWG shows a large extent of flexibility and it is tolerant to fabrication inaccuracies. All of these positive features result in AWG being considered as one of the most promising technologies in FBG based sensors interrogation. There have been a couple of methods set forth to employ this device as a part of sensing mechanism in reality and the research is still going on ([2] and [3]).

In addition to all of the aforementioned positive features of the AWG, its capability of interrogating several signals simultaneously has acted as a strong motivation to employ AWG as an interrogator unit for a TFBG sensor.

This multidisciplinary research consists of two parts. The first part is an attempt to realize the use of TFBG as a refractive index sensor. Designing a suitable AWG to serve as the interrogator unit for the TFBG refractive index sensor is the second goal of this research. In the sensor part, the work has been mostly experimental whereas in the interrogator part we had to limit ourselves to simulation. ${ }^{3}$ The next section of this chapter deals with the structure of the thesis and declares the way it has been organized.

\subsection{Organization of the Thesis}

This thesis reports the results obtained during the course of the author's studies at the Department of Electronics, Carleton University, Canada.

Chapter 2 serves as a summary of the basic concepts involved in the operating principles and design procedure of Arrayed Waveguide Gratings. We review some of

\footnotetext{
${ }^{3}$ This being limited to simulation in the interrogator part of the work, was due to the fact that because of funding issues, the designed AWG had to be fabricated in Canadian Photonics Fabrication Center (CPFC) where they were not ready to take the job because of technical problems.
} 
the fundamental aspects of AWG such as system specifications of the device. We also review crosstalk sources in the device and loss mechanisms. At last, a step by step design procedure is set forth to enable to realize a device exhibiting desired system specifications through playing with geometric parameters.

In Chapter 3, fundamental theories and concepts behind TFBGs have been discussed. It is declared there that introducing a tilt angle in the grating plane of a FBG, will yield the capability of sensing RI of the surrounding environment.

Chapter 4 presents our results on AWG simulation as a sensor interrogator. In this chapter, by examining the transmission spectrum of a TFBG, we will try to come up with a customized AWG design suitable for our application. In order to acquire the optimum design, we will compare several designs with each other and we will discuss advantages and disadvantages of these designs.

Chapter 5 presents our results on applying TFBG as an external material RI sensor. The conducted experiment is demonstrated and results are verified through numerical simulation.

In Chapter 6, we provide a summary of this thesis research, and suggest some directions for future work. 
"La semplicità è la più alta forma di sofisticazione. "1

- Leonardo di ser Piero da Vinci

\section{Chapter 2}

\section{Arrayed Waveguide Grating}

An overall review on arrayed waveguide grating is the main objective of this chapter. This review will include operating principles and design procedure as well. Loss mechanisms and crosstalk sources are other issues that will be discussed in this chapter. The application of AWG as a sensor interrogator will be the concluding subject of the chapter.

\subsection{AWG as a sensor interrogator}

The extraordinary characteristics of FBG sensors such as low cost, small size, capability of multiplexing, ease of fabrication and immunity to electromagnetic interference have caused FBG sensors to be considered as the most promising sensing technology. On the other hand, the inherent wavelength-encoded operation of FBG sensors makes them even more attractive due to the fact that wavelength is an absolute parameter and is not dependent on losses of the system and source power fluctuations. In order to recover the wavelength-encoded information provided by the FBG sensor, one needs to develop an interrogation scheme capable of tracking changes in the center wavelength of the light reflected back by FBG. It may come to the mind that utilizing some laboratory equipments such as optical spectrum analyzer would solve this

\footnotetext{
1 "Simplicity is the ultimate form of sophistication."
} 
problem. However this is not feasible in field applications due to the large size, heavy weight and constant need of calibration.

In general to be utilizable in field applications, an interrogator unit should possess characteristics such as portability, low cost, high measurement accuracy, high measuring speed, high resolution, large measurement range and capability of multiplexing. None of the traditionally used methods such as bulk optical edge filter [4], scanning Fabry-Perot filters [5], wavelength division fiber couplers [6], acousto-optic tunable filters [7] and MZ interferometers [8], satisfy all of the above mentioned requirements.

Recently utilizing arrayed waveguide gratings (AWG) as a spectrum analyzer, which fulfills all the requirements of an ideal interrogator, has attracted enormous interest. Using AWG as a sensor interrogator enables not only tracking the shifts in the wavelength but also tracking the changes in the amplitude of the light coming from the sensor which in some applications is a key feature. Several interrogation schemes have been explored in the literature with various advantages and disadvantages. Later on, we will discuss the fundamentals of these schemes ([2] and [3]).

\subsection{Operating Principles}

Figure 2.1 shows a schematic layout of an arrayed waveguide grating $\left(\mathrm{PHASAR}^{2}\right)$. An AWG consists of two Free Space Couplers(FSC) or Free Propagation Regions which are connected through the arrayed waveguide grating. The light enters the device via the input port and it propagates in the free space coupler. As it is not confined laterally anymore in the free space coupler, the light diverges while propagating. At the end of FSC the light is coupled into the arrayed waveguides. Arrayed waveguides are designed in such a manner that the optical path difference between two adjacent

\footnotetext{
${ }^{2}$ The terms AWG and PHASAR may be employed interchangeably in this thesis. Please notify that they both refer to the same device.
} 


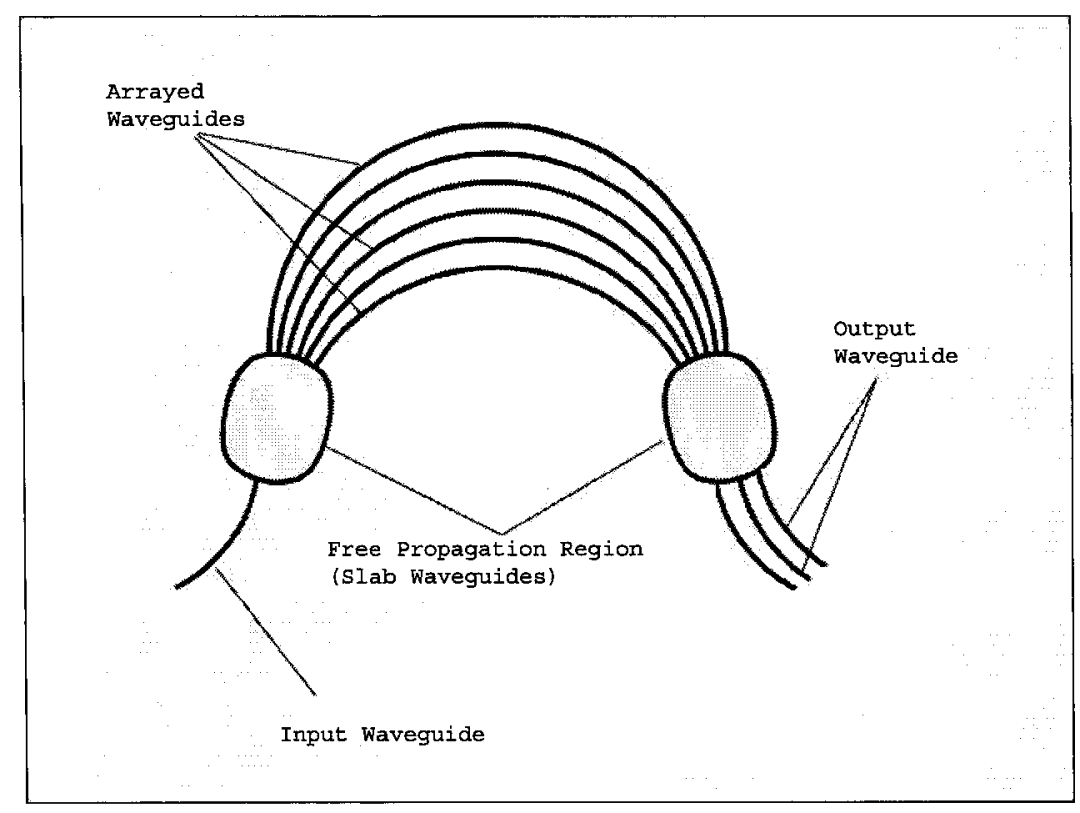

Figure 2.1: A schematic layout of an AWG, Different parts of the device such as input waveguides, arrayed waveguides, free propagation region and output waveguides are marked on the picture.

waveguides equals an integer multiple of $2 \pi$ for the center wavelength. For this particular wavelength (center wavelength) the phase fronts arrive at the end of arrayed waveguides with equal phase (apart from an integer multiple of $2 \pi$ ) and the field distribution at the end of first FSC (input aperture) is reproduced here (output aperture) once more with the same amplitude and phase distribution and an image of the input field at the object plane will be reproduced at the image plane.

The PHASAR is dispersive in the sense that a deviation from center wavelength will cause a linear variation in phase change in the output aperture, thus the outgoing wave will tilt and the focal point will shift along the output or image plane. This dispersion is due to the constant path difference between adjacent waveguides. By placing receiver waveguides in appropriate places in the image plane, spatial separation of the multiple wavelength channels is obtained. 


\subsection{Grating Equation}

The tilt angle, $\Theta$, along which a particular beam of light with wavelength $\lambda$ concentrates, can be determined by the use of grating equation[9]:

$$
N_{s} d_{a} \sin \Theta+N_{g} \Delta \mathrm{E}=m \lambda
$$

where $N_{s}$ is the effective refractive index of the slab (free propagation regions), $d_{a}$ is the separation between arrayed waveguides, $N_{g}$ is the effective refractive index of the waveguides in the grating and also the effective refractive index of the input and output waveguides and $\Delta \mathrm{E}$ is the path difference between two adjacent waveguides in the grating. In order to obtain the right focusing, the length difference, $\Delta \mathrm{L}$ should be chosen equal to an integer number of wavelengths, measured inside the arrayed waveguide[1] as follows:

$$
\Delta \mathrm{E}=m \cdot \frac{\lambda_{c}}{N_{g}}
$$

By differentiating equation (2.3.1) the angular dispersion of the PHASAR is achieved. Angular dispersion is the relationship between wavelength and diffraction angle(Figure 2.2). This relationship is achieved by differentiating of (2.3.1) with respect to $\lambda$. One should be aware that $N_{g}$ changes with $\lambda$. This change is caused by chromatic dispersion of the waveguide material and waveguide structural dispersion. Using $\Theta \approx \sin \Theta$ and differentiating (2.3.2), the resultant angular dispersion is:

$$
\frac{d \Theta}{d \lambda}=\frac{m \widetilde{N_{g}}}{N_{s} d_{a}}
$$

where $\widetilde{N_{g}}$ equals $1-\left.\frac{\lambda}{N_{g}} \frac{d N_{g}}{d \lambda}\right|_{\lambda_{c}}$. 


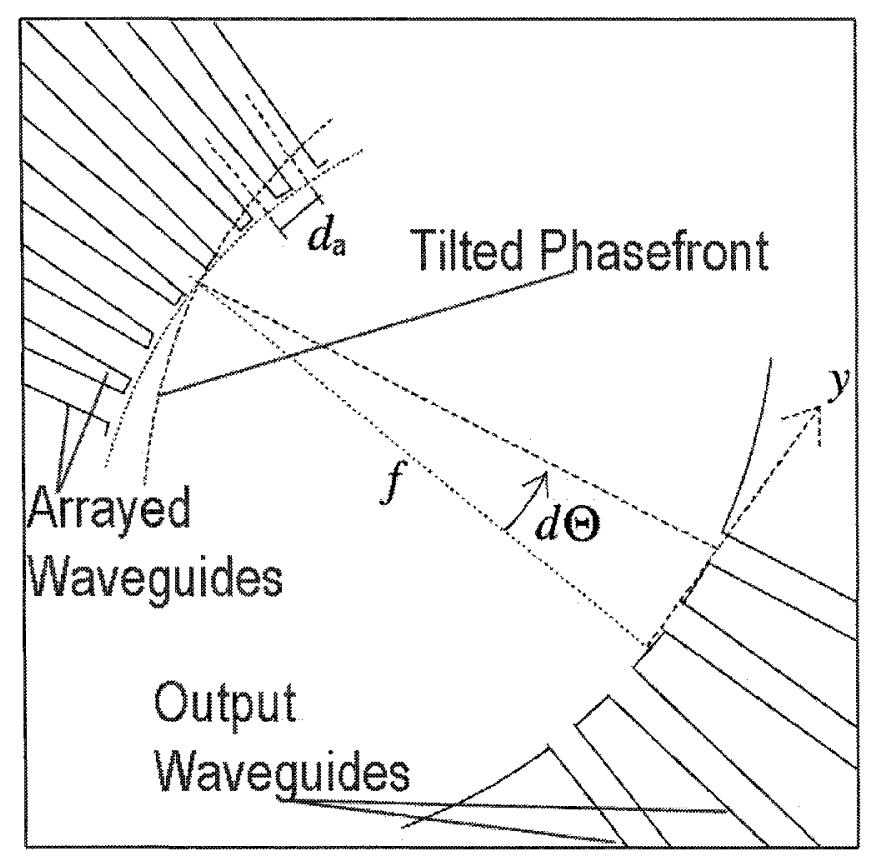

Figure 2.2: Deviating from the operating wavelength $\lambda_{c}$, tilts the wave front at the output aperture and therefore the focal point will shift along the image plane.

\subsection{System Specifications of AWG}

For Arrayed Waveguide Gratings (AWG) to be used as a part of an optical network or to be operated in other applications, such as sensor interrogators, there are some specifications to be met. These System Specifications(SS) are tabulated in table 1.1. Table 1.2 shows the Geometric Parameters(GP) of an AWG. These Geometric Parameters are our control levers over the System Specifications of the device.

In the following subsections, the relationship between system specifications and geometric parameters will be discussed. 


\begin{tabular}{|c|l|}
\hline System Specification Symbol & Description \\
\hline$\Delta v_{c}$ & Channel Spacing \\
$\Delta v_{b w}$ & Channel Bandwidth(1-dB and 3-dB \\
$\chi_{t}$ & Cross Talk(Adjacent and non-adjacent channels) \\
$\Delta v_{F S R}$ & Free Spectral range \\
$L_{n u}$ & Non-Uniformity Loss \\
$L_{I}$ & Insertion Loss \\
$L_{D}$ & Diffraction Loss \\
$\mathrm{PD}$ & Polarization Dependence \\
$\mathrm{BS}$ & Band-Pass Shape(Gaussian or Flat) \\
\hline
\end{tabular}

Table 2.1: System Specifications of an AWG(detailed descriptions follow).

\subsubsection{Channel Spacing}

The wavelength difference between the transmission peaks of the neighboring output waveguides is called Channel Spacing. A deviation from the operating wavelength will result in the displacement of the focal point along the image plane. For a small change in the wavelength the relationship $\Theta \approx \sin \Theta=d_{y} / f$ holds(Figure 2.2, where $d_{y}$ is the displacement of the focal point along the image plane and $f$ is the focal distance of the slab waveguide. By choosing $d_{y}$ equal to the output waveguide spacing, $d_{o}$, the Channel Spacing would be obtained by[9]:

$$
\Delta \lambda=\frac{N_{s} d_{o} d_{a}}{L_{s} m}
$$

\subsubsection{Channel Bandwidth}

The focal field of the AWG displaces along the image plane(output waveguides)as a result of change in the wavelength. The overlap of this focal field and modal fields of the output waveguides leads to the frequency response of different channels. Assuming an aberration free AWG, that is, an AWG whose focal field is an exact 


\begin{tabular}{|c|l|}
\hline Geometric Parameter Symbol & Description \\
\hline$N$ & Number of Waveguides in the array \\
$N_{o}$ & Number of output waveguides \\
$L_{s}$ & Free Propagation Length(Slab Length) \\
$d_{x}$ & Waveguide Spacing(x=i,o,a) \\
$W_{x}$ & Waveguide width $(\mathrm{x}=\mathrm{i}, \mathrm{o}, \mathrm{a})$ \\
$G_{x}$ & Gap between waveguides $(\mathrm{x}=\mathrm{i}, \mathrm{o}, \mathrm{a})$ \\
\hline
\end{tabular}

Table 2.2: Geometric Parameters of a $A W G$; $i$ stands for input, o stands for output and a stands for array.

replica of the modal field of the output waveguides, and assuming identical input and output waveguides, the transmission frequency response arises from the overlap of the waveguide field and itself, displaced over a distance $\delta y$.

$$
T(\lambda)=\int_{-\infty}^{+\infty} u\left(y^{\prime}\right) u\left(y^{\prime}-\delta y \cdot\left(\lambda-\lambda_{c}\right)\right) d y^{\prime}
$$

where $\mathrm{u}(\mathrm{y})$ is the normalized modal field of the input $\backslash$ output waveguides and $\delta y$ is the relative wavelength dispersion and is calculated with equation (2.3.3) and $\mathrm{d} \theta=\sin (\mathrm{d} \theta)[9]$.

$$
\delta y=\frac{L_{s} \cdot d \theta}{d \lambda}=\frac{L_{s} m \widetilde{N}_{g}}{N_{s} d_{a}}
$$

The L-dB bandwidth of this frequency response, by considering modal fields to be Gaussian for simplicity, can be calculated as follow[1]:

$$
\Delta v_{b w}=0.77 \frac{\omega_{e}}{d_{o}} \Delta v_{c} \sqrt{L}
$$

where $\omega_{e}=\omega_{w g}\left(0.5-\frac{1}{V-0.6}\right)$ and $\omega_{w g}$ is the waveguide width. 


\subsubsection{Channel Crosstalk}

Crosstalk is the amount of light coupled into a waveguide at the nominal wavelength corresponding to adjacent and non-adjacent waveguides in the device. There are several mechanisms contributing to crosstalk, some of which can be kept low by proper design while the others are inevitable due to fabrication process imperfections. By theory, one can show that if the fabrication process is perfect the crosstalk level can be kept as low as $-60 \mathrm{~dB}([10])$, however the lowest crosstalk levels ever achieved are around -35 or $-40 \mathrm{~dB}([1])$. These higher crosstalk levels are caused by random phase errors due to non ideal fabrication process. The most important crosstalk incurring mechanisms are demonstrated in the following.

\section{Output Waveguide Coupling}

Output waveguide coupling is the most obvious source of crosstalk in a PHASAR. The decaying tail of the modal field, especially in weakly guiding structures(i.e. the structures with low refractive index contrast)gets coupled into the neighboring waveguides and raises crosstalk. This type of crosstalk brings about a trade-off between the size of the device and desired crosstalk level. In order to keep the crosstalk level low enough, one needs to have the output waveguides well separated so that the decaying tail of the modal fields of the neighboring waveguides attenuates enough(especially for the two closest adjacent waveguides), having larger output waveguide separation leads to a bigger device. By using a higher refractive index contrast one can reduce the size of the device while keeping the crosstalk level still low[11].

\section{Field Truncation}

Truncation of the field due to the finite array aperture at the end of first slab waveguide is another source of crosstalk. This causes the power to be lost at the 
input section of the array resulting in cross talk for two reasons. First, field truncation acts like filtering out some part of the light spectrum which prevents the light from being regenerated in the image plane exactly as it was at the input array aperture. Truncation leads to increasing the sidelobe level of the focal field at the output aperture. Second, that part of the light which was filtered due to the finite width of the input aperture, will cause noise every where in the device if not being absorbed. Some guidelines to choose a proper array aperture opening are given in [1].

\section{Mode Conversion}

The waveguides in a PHASAR are designed to be single mode. If for any reason (such as a fabrication error) they deviate from being strictly single mode, at the junctions between straight and curved waveguides a first-order mode will be excited along with the fundamental mode. This first-order mode propagates with a propagation constant different from that of the fundamental mode resulting in a ghost image. Due to the difference between the propagation constants of the fundamental and first order modes, this ghost image will appear in a different location than the image caused by fundamental mode and may couple into an undesired output waveguide thus degrading the crosstalk level.

\section{Coupling in the Array}

The phase of the light traveling along the arrayed waveguides can be distorted due to the coupling in the input and output sections of the array. It seems that this kind of coupling does not affect the PHASAR performance strongly[1], however Day et al[12] observed a degradation in crosstalk performance through BPM simulation. 


\section{Phase Transfer Incoherence}

Contrary to all the aforementioned sources of crosstalk, phase transfer incoherence increases with an imperfect fabrication process, in the sense that, path length of the arrayed guides are of the order of several thousands of wavelengths. Deviation in the propagation constant may lead to significant errors in the phase transfer, and, ultimately, to deteriorating the crosstalk performance. People have shown that by phase correction, it is possible to obtain improved crosstalk levels. To name the events that lead to phase error, one could mention deviation in the refractive index due to local variations in composition, film thickness or waveguide width or by inhomogeneous filling in of the gaps near the apertures. There are some other more systematic errors such as discretization in the mask pattern generation that contribute to the crosstalk. Because the layout of waveguides on a wafer is patterned via photomask through photolithography process, the resolution of the photomask has a direct influence on the phase errors on an AWG. Lee et al in [13] showed that a resolution better than $25 \mathrm{~nm}$ is critical to produce an AWG of the crosstalk level of around -30dB. Waveguide length deviation can also introduce phase error in an AWG. A complete analytical work on the impact of the length deviation on crosstalk performance of an AWG can be found in [14].

\section{Background Radiation}

The scattered light out of the waveguides at the junctions or rough waveguides edges, is a factor contributing to cross talk under the name of background radiation. This is specially important in the structures where light is also guided besides the waveguides, e.g. in shallowly etched ridge guides or in waveguide structures on a heavily doped substrate where the undoped buffer layer may also act as a waveguide[1]. In practice, crosstalk is not limited by design but by fabrication process. Devices with crosstalk 


\begin{tabular}{|c|}
\hline CrossTalk Source \\
\hline Output waveguide Coupling \\
\hline Field Truncation \\
\hline Mode Conversion \\
\hline Coupling in the Array \\
\hline Phase Transfer Incoherence \\
\hline Background Radiation \\
\hline
\end{tabular}

Table 2.3: Crosstalk Sources in an $A W G$

levels of $-35 \mathrm{~dB}$ are commercially available and improvement in this figure is mainly a mater of improving fabrication process. A summary of crosstalk sources can be found in Table 1.3.

\subsubsection{Free Spectral Range}

The grating equation (2.3.1) reveals that there are many combinations of orders $(\mathrm{m})$ and center wavelengths that satisfy this equation. After each change of $2 \pi$ in the phase difference between adjacent waveguides, $\Delta \Phi=\beta \Delta \mathrm{E}$, the input field will be imaged at the same output position. The Free Spectral Range(FSR) is the wavelength spacing between such center wavelengths. [1] gives FSR through:

$$
\Delta v_{F S R}=\frac{\lambda_{c}}{m} \frac{N_{g}}{N_{g r}} \approx \frac{\lambda_{c}}{m}
$$

\subsubsection{Loss}

There are several sources of loss in arrayed waveguide grating some of which can be minimized while the others including ones due to fabrication imperfections and material properties are out of our reach. 


\section{Waveguide Loss}

One of the most important sources of loss in AWG is the loss of the waveguides themselves. Roughness of the waveguide is another source of loss in the waveguides and it is completely due to the fabrication process. Bending loss is also an issue in AWG because of the bendings that the waveguides experience in grating section. With a proper design it is possible to reduce bending loss. The confinement of the waveguide can be ascribed to the $\mathrm{V}$-factor of the waveguide:

$$
V=\frac{2 \pi}{\lambda_{0}} \omega_{w g} \sqrt{n_{\text {core }}^{2}-n_{c l a d}^{2}}
$$

where $\omega_{w g}$ is the waveguide width. By increasing the V-factor it is possible to minimize bending loss by choosing waveguide bend radii such that the loss values approach the asymptotic limit of the straight waveguide.

\section{Interface Loss}

Loss occurred at the interface of the FPR and arrayed waveguides are known as the interface loss. The light gets reflected back at the interface of the FPR and arrayed waveguide because of the gap between arrayed waveguides. Some of the light, instead of being coupled into the core of the arrayed waveguides, gets coupled into the cladding of these waveguides and this light usually gets absorbed. This kind of loss can be minimized by increasing the number of waveguides in the grating and decreasing the gap between waveguides. The fabrication process imposes a lower limit on this spacing between the waveguides in the grating. There is also another place in where interface loss takes place and it is the transition between FPR and input/output waveguides. 


\section{Spillover Loss}

When designing an AWG it is necessary to have enough number of waveguides in the grating section in order to collect all of the light diverged in the free propagation region, otherwise some of the light will be lost and some of the light will be illuminating regions out of the grating region, this light contributes to crosstalk.

\section{Non-Uniformity Loss}

The far-field intensities of the individual waveguides reduces away from the central waveguide. One can observe loss by moving from the central output waveguide to the lateral ones. This loss is termed Non-Uniformity Loss. A trick to eliminate this loss as much as possible is to design the device for a number of channels larger than the desired number of channels and then only use the central ones.

\section{Insertion Loss}

Of several mechanisms involved in the AWG loss, coupling from fiber to the input waveguides and from the output waveguides to the fiber, loss associated with the propagation in the waveguides and coupling from the free propagation regions to the waveguides are the mechanisms that give raise to insertion loss.

\section{Diffraction Loss}

This type of loss is caused by spatial diffraction to other diffraction orders on the output plane.

\section{Return Loss}

Return Loss is defined as the part of light that is reflected into the input fiber and is considered an important parameter for system performance. This loss is generated at 


\begin{tabular}{|c|}
\hline Loss Mechanism \\
\hline Waveguide Loss \\
\hline Interface Loss \\
\hline Spillover Loss \\
\hline Non-Uniformity Loss \\
\hline Insertion Loss \\
\hline Diffraction Loss \\
\hline Return Loss \\
\hline Bending Loss \\
\hline
\end{tabular}

Table 2.4: Loss Mechanisms in an $A W G$

every optical interface. In $\mathrm{SiO}_{2} / \mathrm{Si}$ material system, this type of loss is generally low due to the low refractive index contrast of the system. The main parts of the device contributing to return loss are fiber to chip and slab to waveguide array interfaces.

Table 1.4 summarizes all of the loss mechanisms in an AWG.

\subsection{Design Procedure}

System specifications such as number of channels, central frequency, channel spacing, L- $d B$ bandwidth, free spectral range, maximal insertion and non-uniformity loss, minimal crosstalk level and polarization dependence are the usual specifications that a designer tries to achieve by playing with Geometric Parameters of an AWG.

Having several geometric parameters and their being interconnected with several system specifications, gives AWG many degrees of freedom in design. The step by step procedure we adapted is based on the procedure explained by [1] as follow:

- Output Waveguide Spacing $d_{o}$ : Desired crosstalk level puts a lower limit on output waveguide spacing. The greater the spacing, the lower crosstalk level, the bigger device. 
- Array Waveguide Spacing $d_{a}$ : This parameter is usually taken equal to the smallest value that fabrication process allows. As we shall see later on, in order to get different slab lengths this parameter is one of the parameters we can play with

- Channel Spacing $\Delta v_{c}$ or $\Delta \lambda$ : This parameter is usually given to us as one of the requirements to be met. As we shall see later on this chapter, channel spacing depending on the application(telecommunication or sensor interrogator) can assumes different values.

- Free Spectral Range $\Delta v_{F S R}$ : Once the number of channels and channel spacing are determined, FSR is actually determined automatically because in practice $F S R=N_{c h} \cdot \Delta \lambda$

- Grating Order $m$ : By central wavelength and FSR being chosen, $m$ is simply determined through (2.4.5) as follow:

$$
m \leq \frac{\lambda_{c}}{F S R}
$$

where $\lambda_{c}$ is the central wavelength and FSR is free spectral range.

- Length of the Slab Waveguide $L_{s}$ : The free propagation region or slab length is obtained through (2.5.2)

$$
L_{s}=\frac{N_{s} d_{o} d_{a}}{m \Delta \lambda}
$$

where $N_{s}$ is the refractive index of the slab waveguide and $\Delta \lambda$ is the channel spacing.

- Number of Waveguides in Array $N$ : is typically chosen equal to 181 or 201 . As a rule of thumb the number of waveguides in the array should be larger than 
four times the number of channels. The larger the number of waveguides in the array, the lower the level of cross talk.

- The length increment $\Delta L$ : is obtained through (2.5.3)

$$
\Delta \mathrm{E}=\frac{m \lambda_{c}}{N_{g}}
$$

where $N_{g}$ is the waveguide effective index in the array and input/output section.

- Half Angle Aperture Opening $\theta_{a}$ : is obtained by the use of (2.5.4)

$$
\theta_{a}=\frac{(N-1) d_{a}}{2 L_{s}}
$$

where $\mathrm{N}$ is the number of waveguides in the array.

In order to give an idea how these equations work, a sample design will be discussed here. In order to get a crosstalk level below $-30 \mathrm{db}$, one should choose $d_{o}=25 \mu \mathrm{m}$ and $d_{a}=12 \mu \mathrm{m}$. These are two typical number, in order to get different designs one can play with these numbers. In [1] there are some criteria to chooses suitable numbers in order to get desired crosstalk levels. The rest of the design process will be as follow:

- $N_{s}=1.4558408$ is given to us by fabrication people.

- $N_{g}=1.454366$ Calculated by the Effective Index Method by WDM-PHASAR software from Opti-Wave.

- $\lambda_{c}=1540.61 \mathrm{~nm}$, This is given to us or could be chosen based on the application.

- $d_{o}=25 \mu \mathrm{m}$.

- $d_{a}=12 \mathrm{~nm}$.

- $\Delta \lambda=0.4 \mathrm{~nm}$, A typical telecom value. 


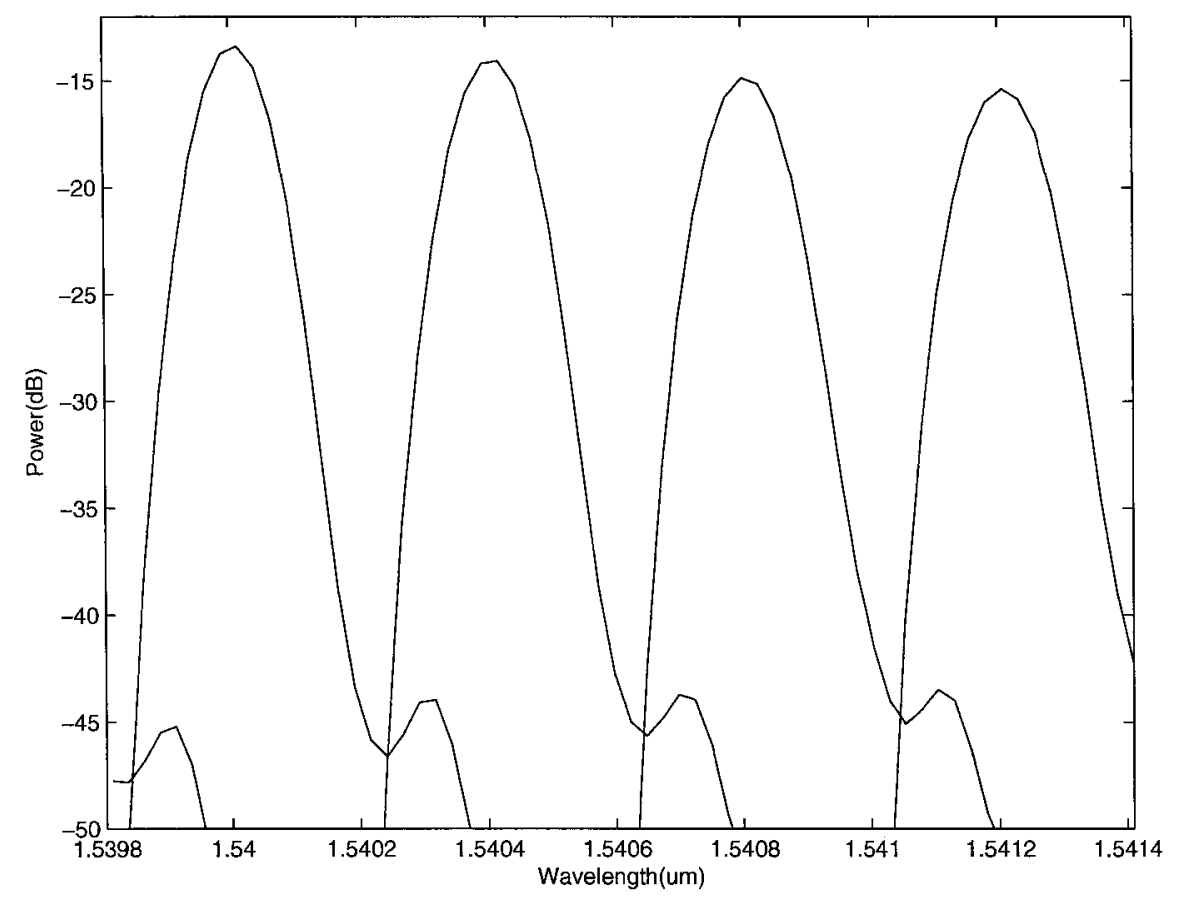

Figure 2.3: $A W G$ output response

- $N_{c h}=43$.

- $\mathrm{FSR}=N_{c} h . \Delta \lambda=43 \times 0.4=17.2$.

- $\mathrm{m}=89$ from $(2.5 .1)$.

- $L_{s}=12268.32135$ from (2.5.2).

- $N_{a}=181$ typical number.

- $\Delta L=94.27767996 \mu \mathrm{m}$ from (2.5.3).

This device has been simulated by OptiBPM software, WDM PHASAR. Figure 2.3 illustrates the output response of the AWG obtained by BPM simulation.

The $3-\mathrm{dB}$ bandwidth of this design is about $0.11 \mathrm{~nm}$, which is quite narrow, the adjacent crosstalk level is below $-30 \mathrm{~dB}$ while the non-adjacent crosstalk level or noise floor is around $-60 \mathrm{~dB}$. There are some techniques to make the bandwidth of the device 
wider and to get a lower crosstalk level[15,16]. It is obvious from the picture that the shape of the pass-band is Gaussian. Getting a flat shape pass-band is desirable in telecom applications and is discussed in [17].

\subsection{Fabrication tolerance}

In order to study the effect of fabrication imperfections on the performance of an AWG, one should employ statistical methods since the errors are of a random nature and are not deterministic. In [18] there is a detailed study of performance degradation due to fabrication errors in AWGs. The random errors occurring during fabrication may affect crosstalk. The transfer function of a conventional AWG is of Gaussian shape and ideally has very low side lobe levels around $-60 \mathrm{~dB}$. Such a low side lobe level would mean a very low crosstalk exhibited by AWG. It has been noted that the origin of crosstalk in AWG is mainly due to the phase errors between the different arms of the grating and these phases errors could be a result of inaccuracy in the waveguide geometry or the refractive index value inexactitude.

Several types of errors may occur during the fabrication process of an AWG due to imperfections of the fabrication technology. The most important ones are deviation of the value of refractive index from the original value used in design stage and waveguide core size inaccuracy. Deviating from the exact value of refractive index leads to the displacement of the central wavelength of output channels. This means a channel that used to appear in the neighborhood of for example, a wavelength of $\lambda_{1}$, now may appear at the wavelength of $\lambda_{1} \pm \Delta \lambda_{1}$. The reason of this shift in the central wavelength of the output channels is straightforward and can be explained as follows. The phase front tilt angle at the end of array waveguides, which is directly proportional to the phase difference accumulated in arrayed waveguides, by changing

the refractive index of the waveguides will change and thus the focusing point of 


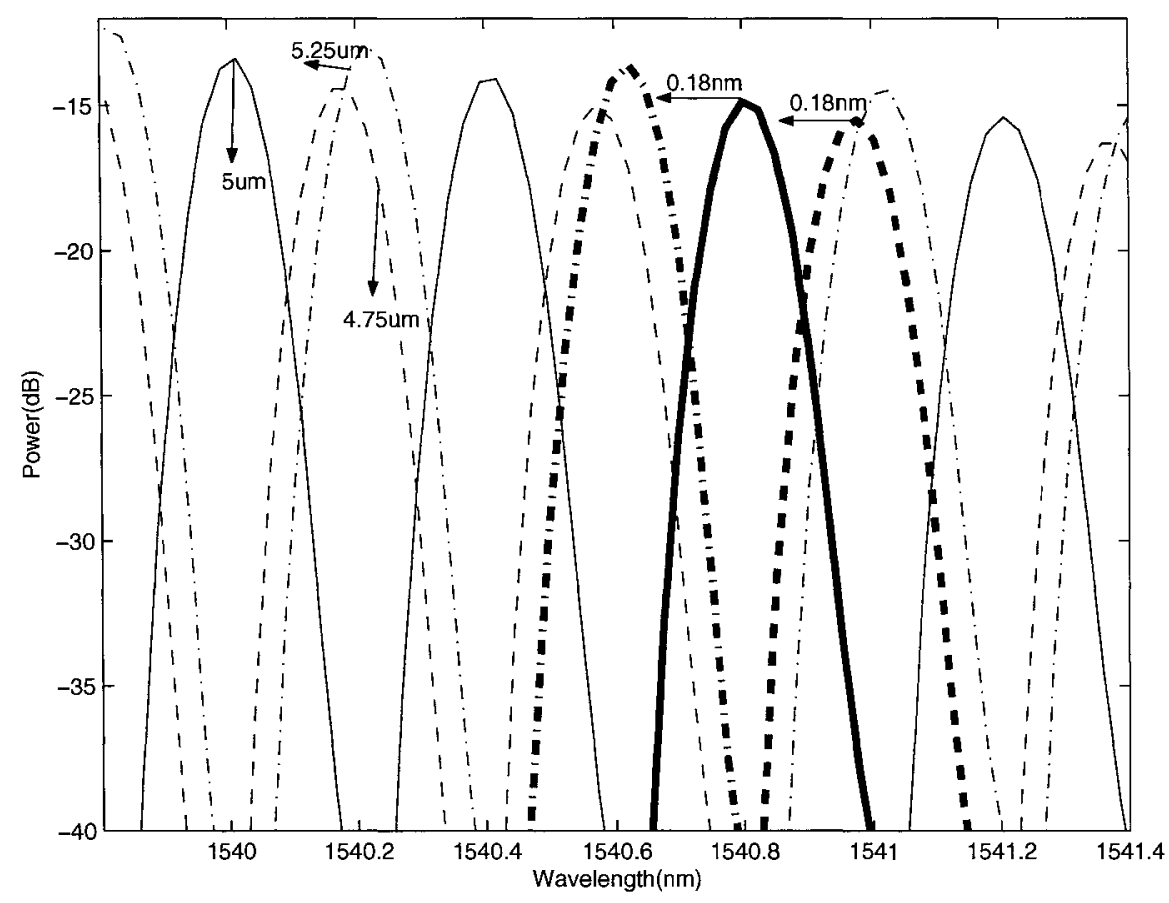

Figure 2.4: Fabrication imperfections: core size inaccuracy

the optical field will shift along the output plane of the AWG. Departing from the precise value of the waveguide core size may also result in the degradation of the performance of AWG. The most visible ones of these performance degradations would be a slight change in the output channels central wavelength and bandwidth of the output channels. It may also affect crosstalk. The change in the core size leads to change in the effective refractive index of the waveguide which in turn leads to slight change in the focusing point at the end of second slab waveguide.

Figures 2.4 and 2.5 show the results of a simulation performed in an extreme pessimism, that is, when the waveguide core size or desired refractive index value of the device is completely off all over the place.

Figure 2.4 shows the results for 3 simulations performed by OtptiMPM WDM PHASAR software. The desired waveguide core size is $5 \mu \mathrm{m}$ (solid line) while the simulation is also performed for two other cases where core size is $4.75 \mu \mathrm{m}$ (dashed 


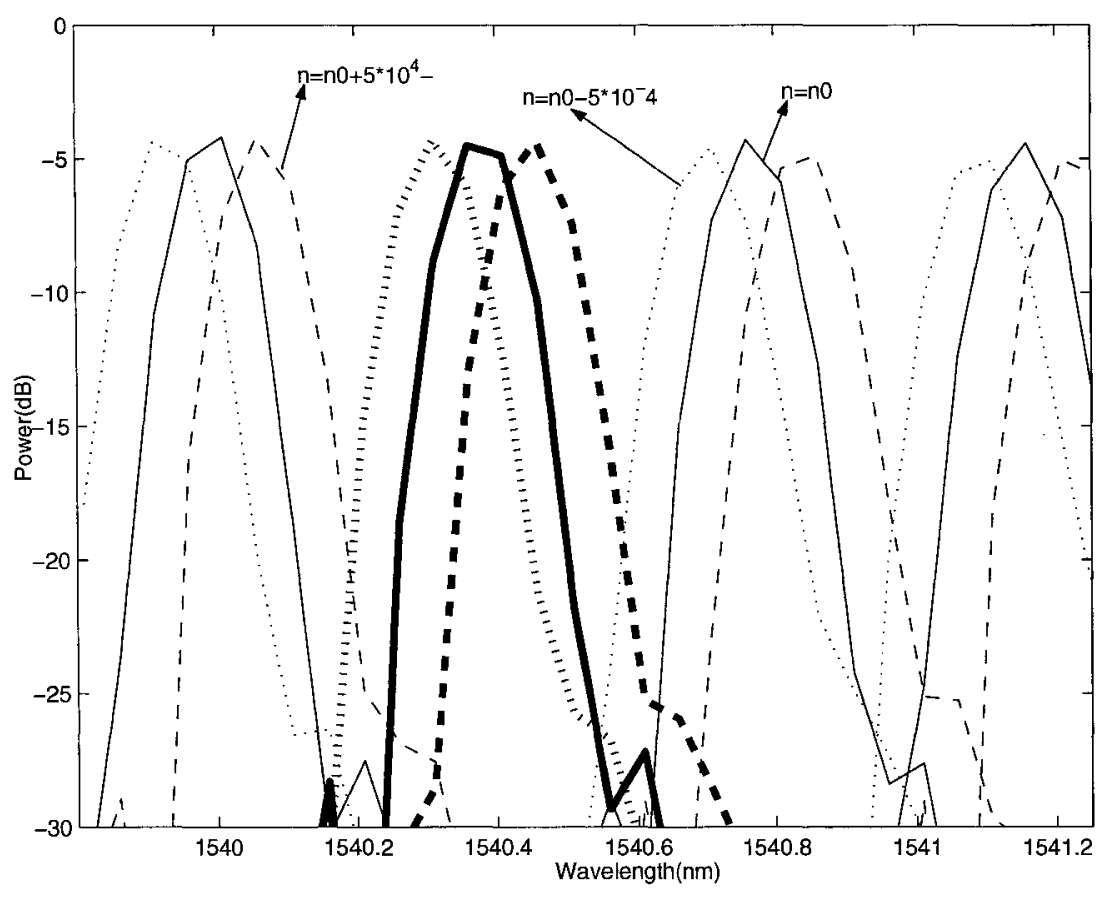

Figure 2.5: Fabrication imperfections: refractive index deviation

line) and $5.25 \mu \mathrm{m}$ (dash-dot line). It is clear from the picture that, a deviation of $0.25 \mu \mathrm{m}$ in the core size causes a displacement of $0.18 \mathrm{~nm}$ in the position of output channel. The other specifications of the transmission response of the AWG, such as 3-dB bandwidth $(0.11 \mathrm{~nm})$, crosstalk(below $-30 \mathrm{~dB})$ and channel spacing $(0.4 \mathrm{~nm})$, all remain approximately the same which indicate the AWG design is quiet tolerant to fabrication errors for sensor interrogator applications since the exact position of $\lambda_{c}$ is not critical.

The results of the effects of deviation from desired refractive index value on the performance of AWG are shown in Figure 2.5. Again, one can easily observe that the displacement of the output channel is the main effect of the of refractive index change. 


\subsection{Interrogation}

Despite the fantastic features exhibited by FBG optical sensors, this sensor technology is not the dominant technology in the sensor market. In order to find the reason for this technology's not being comprehensive, one should explore the interrogator head of the sensor system. There have been many interrogation schemes developed in order to decode the wavelength encoded information coming out of an FBG sensor. Most of these schemes suffer from being dependent on expensive and/or bulky components which make them unsuitable for field applications. The compact size and passive nature of the AWG, potential of multiple functionality on a single chip, i.e., integration of multiple AWGs, detectors, optical switches, signal processing and controlling electronics, etc; high speed operation and stability due to the solid, monolithic, nature of the device and potential for volume production, hence the reduction in cost, are all the factors that have caused AWG to attract a huge attention to be used as an interrogator. In order to give a perspective on how an AWG is employed as an interrogator, two of the most popular methods of employing an AWG as interrogator will be discussed here. One of these methods relies on the taking the ratio of the intensities in adjacent AWG channels[19] while the other one involves thermally scanning of the AWG transmission spectrum in order to get the maximum power in the output detector[3].

In order to give a more detailed explanation on these two methods, one can approximate the reflection spectrum of an FBG as follow:

$$
B_{i}(\lambda)=b_{i} \exp \left[-4(\ln 2) \frac{\left(\lambda-\lambda_{b i}\right)^{2}}{\Delta \lambda_{b i}^{2}}\right]+b_{0 i}
$$

where $b_{i}$ and $\Delta \lambda_{b i}$ are the peak reflectance and FWHM of the Gaussian profile of the $i$ th FBG in a series of multiplexed FBGs, respectively. $b_{o i}$ is called the overall noise floor of the FBG or the bias component. On the other hand, the receiving function, or spectral transmittance of each channel of AWG can also be well approximated by 
a Gaussian form.

$$
A_{m}(\lambda)=a_{m} \exp \left[-4(\ln 2) \frac{\left(\lambda-\lambda_{a m}\right)^{2}}{\Delta \lambda_{a m}^{2}}\right]+a_{0 m}
$$

where $a_{m}$ and $\Delta \lambda_{a m}$ are the peak transmittance and FWHM of the Gaussian component of the $m$ th output channel of the AWG, respectively. Again here $a_{0 m}$ is the noise floor of this transmittance function caused by stop band transmittance of each output channel.

\section{Wavelength Interrogation}

Sano and Yoshino[19], consider the interrogation of N FBGs with central wavelengths $x_{i}=\lambda_{b i}(1 \leq \mathrm{i} \leq \mathrm{N})$. In order for the wavelength to be interrogated uniquely, the FSR of the AWG has to be wider than spectral width, $\Delta \lambda_{s}$, of the input light source. Thus the output signal from the $m$ th channel of the AWG would be:

$$
I_{m}\left(\lambda_{b i}\right)=d_{m} \int_{0}^{\infty} S(\lambda)\left[\sum_{k=1}^{N} F_{k}^{2} B_{k}(\lambda)\right] A_{m}(\lambda) d \lambda+I_{F m}
$$

$\rho\left(x_{i}\right)$ is called the wavelength interrogation function and is defined as follow:

$$
\rho\left(x_{i}\right)=\log \frac{I_{m+1}\left(x_{i}\right)}{I_{m}\left(x_{i}\right)}
$$

(2.7.4) takes zero at $x_{i}=\left(\lambda_{a, m+1}+\lambda_{a m}\right) / 2$, which is the the middle wavelength of the adjacent two channels of AWG. Sano and Yoshino show that (2.7.4) is in linear relationship with FBG's central wavelength $x_{i}$, therefore $x_{i}$ can be determined readily from the measurement of the $\rho\left(x_{i}\right)$.

This simple approach yields good performance but suffers from a limited usable range (less than channel spacing) and a reduced sensitivity near the extremes of the range. Norman et al[2] overcame these drawbacks by employing a heterodyne approach based on interferometric wavelength shift detection. Although Norman's 
approach resolved the drawbacks of Sano's and Yoshino's, it suffers from making the interrogation system much more complicated.

Xiao et. al. in National Research Council of Canada, proposed another interrogation scheme which is based on the idea that the transmittance response of an AWG output channel can be tuned to the sensor wavelength. Thus one might be able to correlate the temperature of the AWG to the sensor wavelength. In order to simplify the relations, without loosing generality, one can take (2.7.1) and (2.7.2) and neglect the noise floor terms, to serve as the FBG reflectance and AWG output channel transmittance response. Hence, the power detected by $n$th channel of AWG can be described as:

$$
I_{n i} \approx k_{n} A_{n} S_{i} \Delta \lambda_{A n} \Delta \lambda_{S i} \times \sqrt{\frac{\pi}{\left(\Delta \lambda_{A n}^{2}+\Delta \lambda_{S i}^{2}\right)}} \times \exp \left[-4(\ln 2) \frac{\left(\lambda_{A n}-\lambda_{S i}\right)^{2}}{\Delta \lambda_{A n}^{2}+\Delta \lambda_{S i}^{2}}\right]
$$

where $k_{n}$ is the constant representing the source power, detector sensitivity, etc. It is obvious from this equation that $I_{n i}\left(\lambda_{A n}\right)$ curve is a Gaussian function with a FWHM of $\sqrt{\Delta \lambda_{A n}^{2}+\Delta \lambda_{S i}^{2}}$ and the peak value of:

$$
K_{n}=k_{n} A_{n} S_{i} \Delta \lambda_{A n} \Delta \lambda_{s i} \times \sqrt{\left(\frac{\pi}{\Delta \lambda_{A n}^{2}}+\Delta \lambda_{S i}^{2}\right) 4 l n 2}
$$

Peak value is achieved when $\lambda_{A n}=\lambda_{S i}$. It has been observed that AWG channel wavelength $\lambda_{A n}$ changes linearly with temperature[3] as follow:

$$
\lambda_{A n}(T)=B \cdot T+C
$$

where $\mathrm{B}$ and $\mathrm{C}$ are constants respectively determined from measurement and $\mathrm{T}$ is temperature. The value of B measured in NRC by Xiao and his colleagues was 0.011 $\mathrm{nm} / C^{\circ}$. The value of $\mathrm{C}$ depends on the AWG channel number. From (2.7.5), (2.7.6) and (2.7.7), we have:

$$
I_{n i}(T)=K_{n} \exp \left[-4(\ln 2) \frac{\left(B . T+C-\lambda_{S i}\right)^{2}}{\Delta \lambda_{A n}^{2}+\Delta \lambda_{S i}^{2}}\right]
$$

(2.7.8) reveals that by finding the temperature corresponding to the peak of the $I_{n i}(T)$ curve, one can obtain the sensor wavelength $\lambda_{S i}$. 


\subsection{Conclusion}

Arrayed waveguide gratings were studied as one of the most promising devices, capable of fulfilling the requirements of an ideal sensor interrogator. Operating principles were discussed and a step by step design procedure was introduced to realize an AWG that satisfies specified system characteristics such as channel spacing, crosstalk level and bandwidth. Studying the different loss mechanisms an AWG suffers from, was another part of this chapter. The rest of the chapter was organized to investigate the sources from which cross talk springs and fabrication tolerances of the AWG. At the end, two different methods of interrogation using AWG were presented. 
"There is nothing worse than a brilliant image of a fuzzy concept"

- Ansel Adams

\section{Chapter 3}

\section{Tilted Fiber Bragg Grating: Concepts and Theory}

In this chapter, tilted fiber Bragg gratings will be addressed as a special type of ordinary fiber Bragg gratings. First, fiber Bragg gratings as a family of short-period gratings will be introduced and their properties explored through coupled mode theory. The sensitivity of FBGs to temperature and strain will be the subject of investigation and an effort will be made to derive the mathematical relationships, relating the Bragg center wavelength to the temperature and/or the strain applied on the fiber. In the second part of the chapter TFBGs will be addressed specifically. Their properties will be discussed and the effect of introducing a tilt angel, $\theta_{t}$, on the properties of an ordinary FBG spectrum will be explained. Experimental examples are also employed wherever needed to clarify the subject.

\subsection{Simple Bragg grating}

A Fiber Bragg Grating(FBG) in its simplest form consist of a periodic modulation of refractive index in the core of a single mode fiber where the grating planes are perpendicular to the longitudinal axis of the fiber. These grating planes scatter the light. If the Bragg condition is satisfied, the back scattered light will interfere 
constructively to form a backward propagating peak with a center wavelength defined by Bragg grating.

The Bragg grating condition is basically the requirement that satisfies both the energy and the momentum conservation. The energy conservation $\left(\hbar \omega_{i}=\hbar \omega_{r}\right)$ to be satisfied requires the frequency (wavelength) of the reflected light be the same as the incident light while the momentum conservation requires the wavevector of the incident light $\mathbf{k}_{\mathbf{i}}$, plus the wavevector of the of the grating $\mathbf{K}$ equal the wavevector of the scattered light $\mathbf{k}_{\mathbf{r}}$.

$$
\mathbf{k}_{\mathbf{i}}+\mathbf{K}=\mathbf{k}_{\mathbf{r}}
$$

where grating wavevector, $\mathbf{K}$, has a direction perpendicular to the grating planes with a magnitude of $2 \pi / \Lambda(\Lambda$ is the periodicity of the grating). For pure reflection, the diffracted wavevector is equal in magnitude, but opposite in direction, to the incident wavevector. Thus (3.1.1) becomes:

$$
2\left[\frac{2 \pi n_{e f f}}{\lambda_{B}}\right]=\frac{2 \pi}{\Lambda}
$$

from which Bragg condition could be deduced

$$
\lambda_{B}=2 n_{e f f} \Lambda
$$

In (3.1.3), $\lambda_{B}$ is the free space center wavelength of the input light that will be reflected back from the grating and $n_{\text {eff }}$ is the effective refractive index of the single mode propagating in the core of the fiber[20].

\section{Reflectivity of FBG}

The refractive index profile of a uniform grating within the core of a single mode fiber can be described as[20]:

$$
n(z)=n_{a}+\Delta n \cos \left(\frac{2 \pi z}{\Lambda}\right)
$$




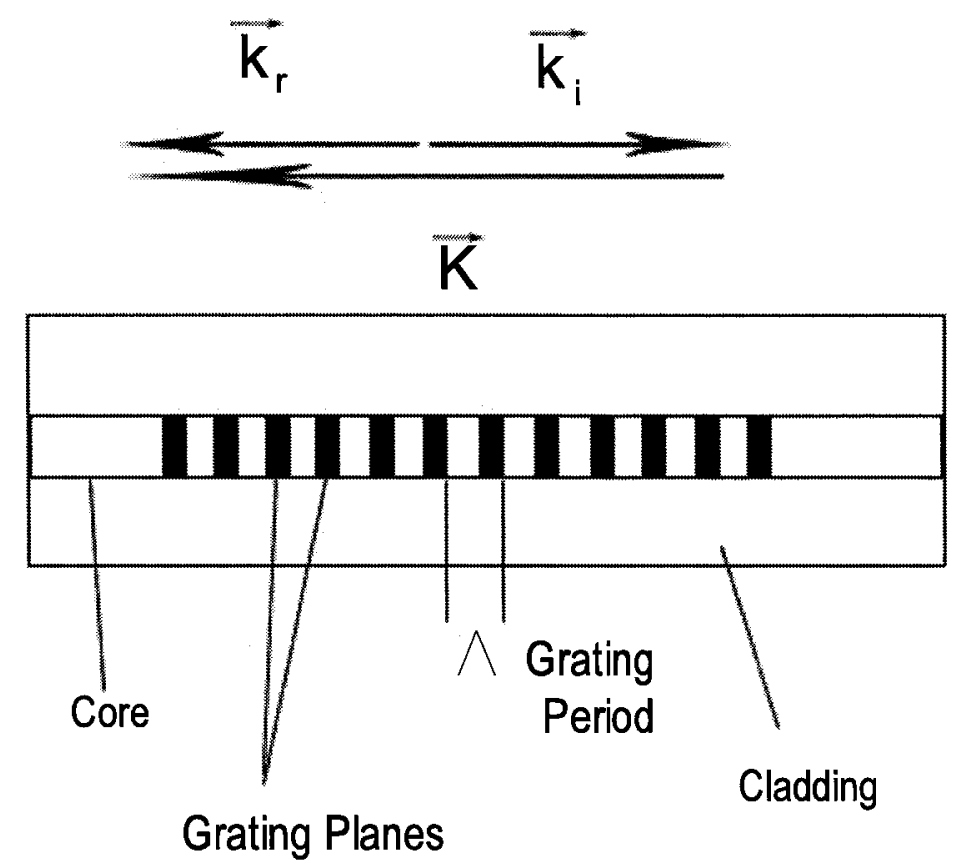

Figure 3.1: Illustration of a uniform Bragg grating. Also shown are incident, reflected and grating wavevectors.

where $\Delta n$ is the refractive index perturbation amplitude with a typical value of $10^{-3}$ to $10^{-4}, n_{a}$ is the average refractive index and $\mathrm{z}$ is measured along the longitudinal axis of the fiber. The local reflectivity is the complex ratio of the forward and backward propagating wave amplitudes. In order to derive the expression of the reflectivity, $r(z)$, one needs first to start with Helmholtz equation[21]

$$
\nabla^{2} \widetilde{E}+\widetilde{n}^{2} k_{0}^{2} \widetilde{E}=0
$$

where $\widetilde{E}$ is the Fourier Transform of the electric field distribution with respect to time.

Since $n(z)$ is periodic in $z$ direction it can be expressed as a Fourier Series as 
follow:

$$
n(z)=\sum_{m=-\infty}^{+\infty} n_{m} \exp [2 \pi i m(z / \Lambda)]
$$

the solutions to (3.1.5), considering both forward and backward propagating waves are of the form of

$$
\widetilde{E}(\mathbf{r} \cdot \omega)=F(x, y)\left[\widetilde{A}_{f}(z, \omega) \exp \left(-i \beta_{B} z\right)+\widetilde{A}_{b}(z, \omega) \exp \left(+i \beta_{B} z\right)\right]
$$

In which $\beta_{B}$ is the Bragg wave number. Assuming that $\widetilde{A}_{f}$ and $\widetilde{A}_{b}$ vary slowly with z and are nearly phased matched terms, the coupled mode equations take the form of:

$$
\begin{aligned}
& \frac{\partial \widetilde{A}_{f}}{\partial z}=i \delta(\omega) \widetilde{A}_{f}+i \kappa \widetilde{A}_{b}, \\
& \frac{\partial \widetilde{A}_{b}}{\partial z}=i \delta(\omega) \widetilde{A}_{b}+i \kappa \widetilde{A}_{f}
\end{aligned}
$$

where $\delta$ is the measure of the detuning from Bragg frequency $\left(\omega_{B}=\pi \mathrm{c} /\left(n_{e f f} \Lambda\right)\right.$.

$$
\delta(\omega)=\left(n_{e f f} / c\right)\left(\omega-\omega_{B}\right) \equiv \beta(\omega)-\beta_{B}
$$

The coupling coefficient, $\kappa$ is the factor governing the grating-induced coupling between forward and backward propagating modes and is expressed as:

$$
\kappa=\frac{k_{0} \iint_{-\infty}^{+\infty} \delta n_{1}(x, y)|F(x, y)|^{2} d x d y}{\iint_{-\infty}^{+\infty}|F(x, y)|^{2} d x d y}
$$

Solving (3.1.8) and (3.1.9), simultaneously with $A_{f}(z=0)=1$ and $A_{b}(z=l)=0$ as boundary conditions for the forward and backward waves at the two ends of the uniform grating of length $l$, and taking the squared values of their ratio, yields the reflectivity ${ }^{1}$ as follow[20]:

$$
R(l, \lambda)=\frac{\kappa^{2} \sinh ^{2}(s l)}{\delta k^{2} \sinh ^{2}(s l)+s^{2} \cosh ^{2}(s l)} .
$$

\footnotetext{
${ }^{1}$ Reflectivity is defined here as the magnitude squared of the amplitude reflection.
} 


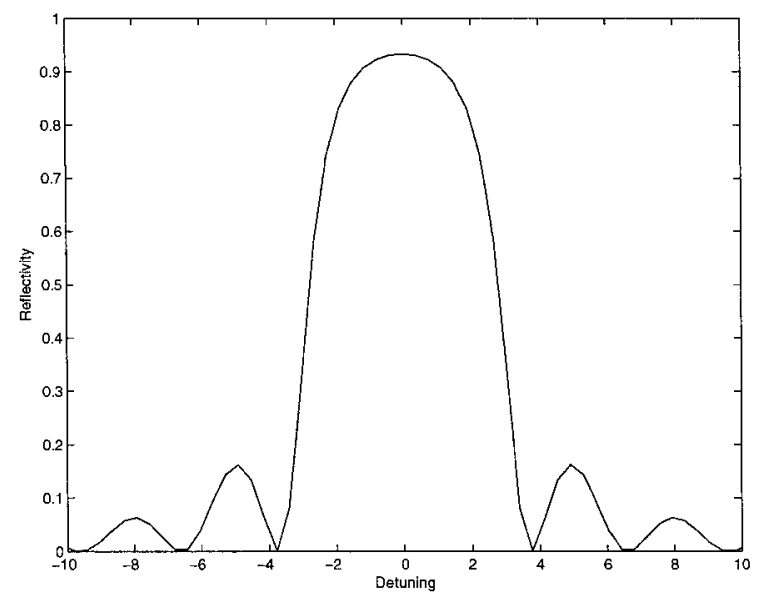

Figure 3.2: Reflection spectrum of a Bragg grating as a function of wavelength detuning.

Equation (3.1.12) reveals that reflectivity is a function of the grating length and wavelength $\lambda . \kappa$ is the coupling coefficient obtained from (3.1.11), $\delta \mathrm{k}$ is the wavevector detuning expressed as $\delta \mathrm{k}=\mathrm{k}-\pi / \lambda$ in which $\mathrm{k}=2 \pi n_{a} / \lambda$ is the propagation constant and $s^{2}=\kappa^{2}-\delta k^{2}$. At the Bragg grating center wavelength there is no wavevector detuning, $\delta \mathrm{k}=0$, therefore, $(3.1 .12)$ is simplified to[20]:

$$
R_{\max }(l, \lambda)=\tanh ^{2}(\kappa l)
$$

For a transversely uniform grating, the coupling coefficient, $\kappa=2 \pi \delta n_{1} / \lambda$. By taking $\delta n_{1}$ as a constant and $k_{0}=2 \pi / \lambda$, the coupling coefficient for a sinusoidal grating similar to (3.1.4) with $n_{a}=0$ and $\delta n_{1}=\Delta n / 2$, will be $\pi \Delta n / \lambda$ (here we assume that the overlap between the fiber mode profile and the cross section of the grating plane, is equal to 1). Figure (3.2) shows a sample spectrum of a FBG. Reflectivities larger than $99 \%$ are achievable in practice. An undesirable feature of Figure 3.2 is the presence of multiple sidelobes at each side of the stop band. The origin of these sidelobes is the weak reflections occurring at the grating ends where the refractive index changes abruptly. Even though the change in the refractive index is around $1 \%$ and less, the reflection at two grating ends form a Fabry-Perot cavity with its own wavelength dependent 
transmission. There are apodization techniques used to remove these sidelobes[22].

\subsubsection{Strain and Temperature Sensitivity of FBG}

The center wavelength of an FBG depends on the effective refractive index of the fiber mode and the physical periodicity of the grating planes. The refractive index of the fiber and periodicity of the grating will be affected by changes in temperature and strain. This is the reason why FBGs are being considered as temperature and strain sensors. The strain response arises due to both the physical elongation of the sensor(which affects the periodicity) and the change in the fiber refractive index due to photoelastic effects, whereas the thermal response arises due to the inherent thermal expansion of the fiber material and the temperature dependence of the fiber refractive index. The shift in the Bragg wavelength due to temperature and strain can be expressed as follow[23]:

$$
\Delta \lambda_{B}=2 n \Lambda\left(\left\{1-\left(\frac{n^{2}}{2}\right)\left[P_{12}-\nu\left(P_{11}+P_{12}\right)\right]\right\} \epsilon+\left[\alpha+\frac{\frac{d n}{d T}}{n}\right] \Delta T\right) .
$$

where $\epsilon$ is the applied strain, $P_{i, j}$ coefficients are the $(i . j)^{\text {th }}$ element of strain-optic tensor, $\nu$ is the Poisson's ratio, and $\alpha$ is the coefficient of the thermal expansion(CTE) of the fiber material. The term $\left\{\left(\frac{n^{2}}{2}\right)\left[P_{12}-\nu\left(P_{11}+P_{12}\right)\right]\right\}$ has a numerical value of $\approx 0.22$; Thus the measured strain response at a constant temperature would be[23]:

$$
\frac{1}{\lambda_{B}} \frac{\partial \lambda_{B}}{\partial \epsilon}=0.78 \times 10^{-6} \mu \varepsilon^{-1}
$$

(3.1.15) reveals that the center wavelength of a FBG shifts $1 \mathrm{~nm}$ per $1000 \mu \varepsilon$ at $1.3 \mu \mathrm{m}$. There exist a relationship similar to (3.1.15) for temperature[23]:

$$
\frac{1}{\lambda_{B}} \frac{\partial \lambda_{B}}{\partial T}=6.67 \times 10^{-6} C^{\circ-1}
$$




\subsection{Tilted Fiber Bragg Grating}

As discussed in the previous section, FBGs with properties such as relatively high sensitivity to temperature and strain have found a variety of applications, including pressure-strain sensors, temperature sensors, micro-bending sensors and etc. In parallel with these applications, there is another class of applications in which people need the sensor to sense the refractive index of surrounding environment. In this type of application insensitivity to temperature is practically desirable. The so-far-proposed devices to achieve this goal have involved combination of gratings in one sensor such as two different types of fiber Bragg gratings[24],two fiber Bragg gratings with different cladding diameters and a long period grating (LPG) with a Bragg grating [25]. The differential sensitivity of the two gratings to temperature and the desired measurand is then used to discriminate between the two.

Tilted Fiber Bragg Gratings, a special type of fiber Bragg grating (shortperiod) in which the grating planes are slanted or blazed with respect to the fiber axis, are considered as an alternative answer to this need. The tilt of grating planes gives them the ability of coupling light from the forward propagating core mode to backward propagating cladding modes, as well as backward core modes, therefore, in the transmission spectrum both a core mode resonance and several cladding mode resonances appear. The cladding mode resonances appear at discrete wavelengths shorter than the core mode Bragg resonance. The cladding modes travel close to the cladding-surrounding medium interface which makes them sensitive to the refractive index of the surrounding environment while the core mode resonance or the Bragg resonance does not sense this external refractive index. This property nominates the TFBG sensors as a suitable choice for chemical applications. 


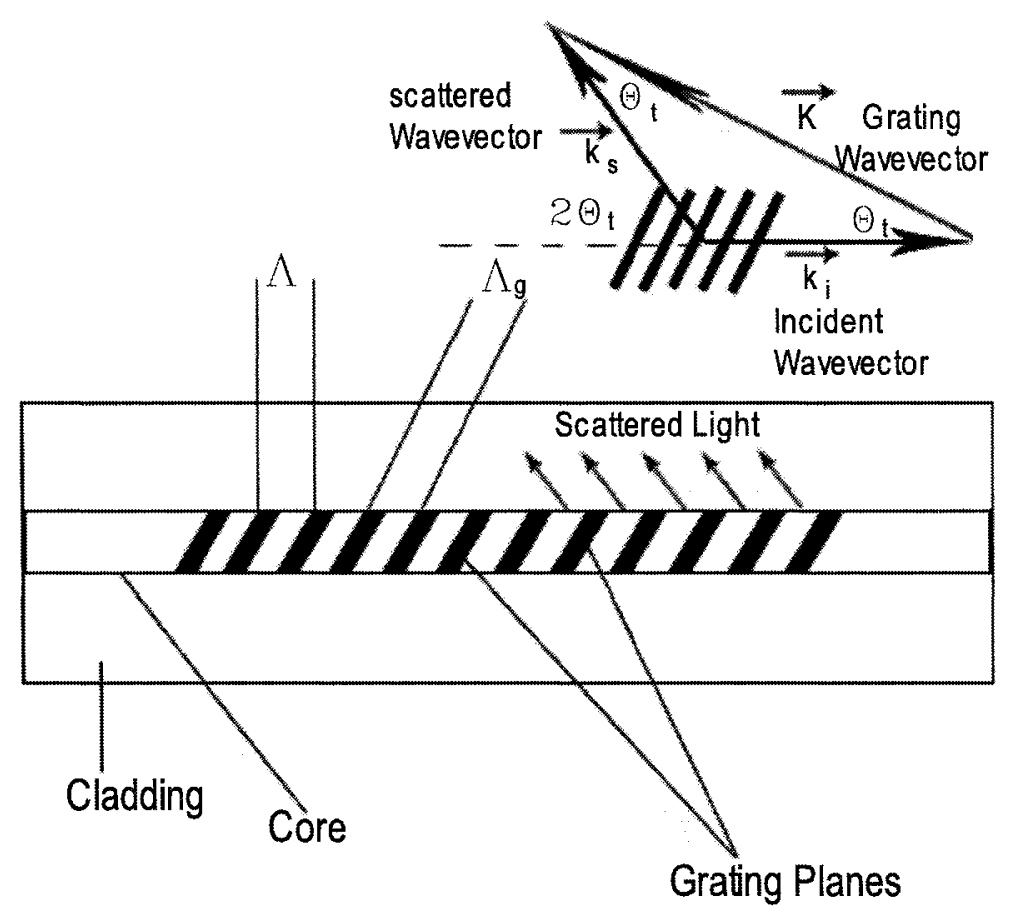

Figure 3.3: Illustration of a Tilted fiber Bragg Grating. Also shown are incident, reflected and grating wavevectors.

\subsubsection{Cladding Mode Coupling in TFBGs}

The coupling efficiency and bandwidth of the light coupled into the cladding depends on the tilt angle, the length and the strength of the refractive index modulation of the grating. Here again, similar to ordinary FBG, both momentum and energy conservation laws should be preserved. Figure 3.3 illustrates the vector diagram of the TFBGs. Analogous to FBG, $\mathbf{K}$ is the tilted grating wavevector, $\mathbf{k}_{\mathbf{i}}$ is the incident light wavevector and $\mathbf{k}_{\mathbf{s}}$ is the scattered light wavevector. Unlike ordinary FBG, here the grating wavevector is incident with an angle $\theta_{t}$ with respect to fiber axis. In order for energy conservation law to be satisfied, the magnitude of the incident light wavevector should be equal to the magnitude of the scattered light wavevector $\left(\left|\mathbf{k}_{\mathbf{i}}\right|=\left|\mathbf{k}_{\mathbf{s}}\right|=k\right)$; For this, the scattered light wavevector should lay at an angle of $2 \theta_{t}$. From the 


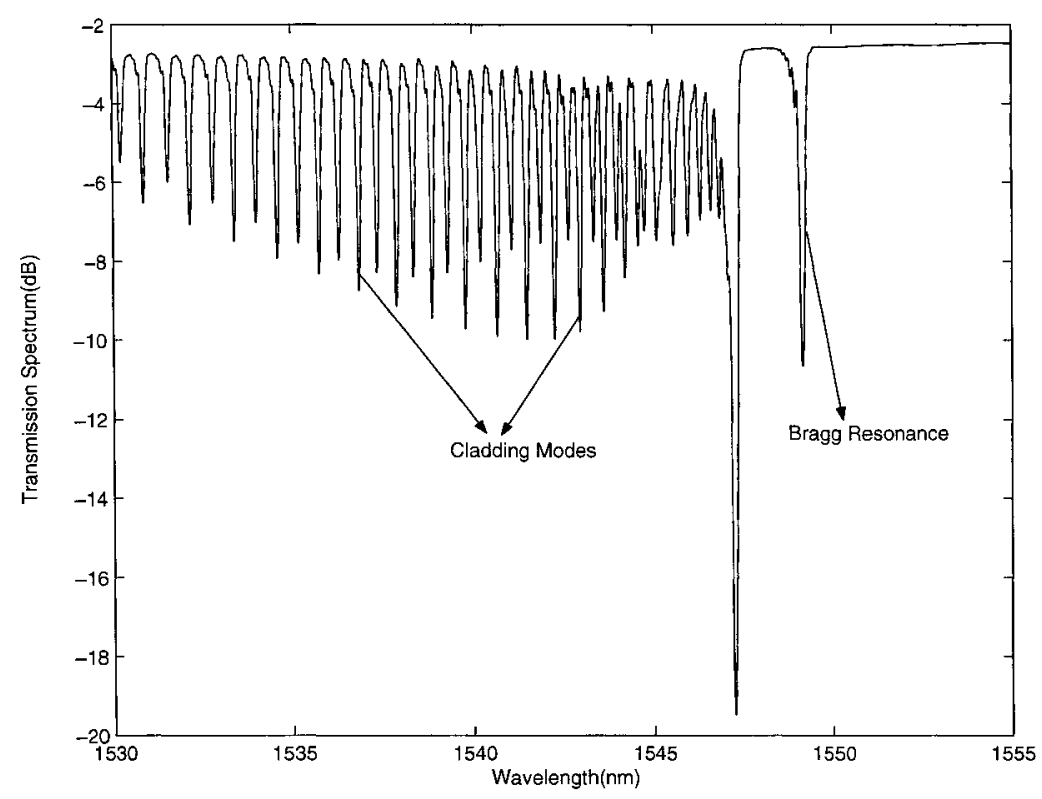

Figure 3.4: A Typical TFBG Transmission Spectrum

wavevector diagram, by applying cosines law we obtain[20]:

$$
\left|\mathbf{k}_{\mathbf{i}}\right|^{2}+\left|\mathbf{k}_{\mathbf{s}}\right|^{2}-2\left|\mathbf{k}_{\mathbf{i}}\right|\left|\mathbf{k}_{\mathbf{s}}\right| \cos \left(\pi-2 \theta_{t}\right)=|\mathbf{K}|^{2}
$$

which simplifies to

$$
\cos \left(\theta_{t}\right)=|\mathbf{K}| / 2 \mathrm{k}
$$

(3.2.2) reveals that the scattering angle is restricted by Bragg wavelength and the effective refractive index. From (3.2.2) one can derive the Bragg wavelength condition of the TFBG as follow:

$$
\lambda_{B}=2 n_{e f f} \Lambda_{g} / \cos \left(\theta_{t}\right)
$$

and cladding mode resonances can be found through[26]:

$$
\lambda_{\text {Clad }, i}=\left(n_{e f f, C l a d, i}+n_{e f f, C o r e}\right) \frac{\Lambda_{g}}{\cos \left(\theta_{t}\right)}
$$

where $n_{e f f, C l a d, i}$ is the effective refractive index for $i^{\text {th }}$ cladding resonance, $n_{e f f, \text { Core }}$ is the core effective index, and $\Lambda_{g}=\Lambda \cos \left(\theta_{t}\right)$ where $\Lambda$ is the periodicity along the fiber 
axis(Figure 3.3).

The wavelength shift of the Bragg resonance and cladding mode resonances due to external refractive index change $\left(\Delta n_{\text {ext }}\right)$ and temperature changes can be derived by differentiating (3.2.3) and (3.2.4) respectively.

$$
\begin{gathered}
\Delta \lambda_{B}=\left[2 \frac{\Lambda_{g}}{\cos \left(\theta_{t}\right)} \frac{\partial n_{\text {eff }, \text { Core }}}{\partial n_{\text {ext }}}\right] \Delta n_{\text {ext }} \\
+\left[2 \frac{\Lambda_{g}}{\cos \left(\theta_{t}\right)} \frac{\partial n_{\text {eff }, \text { Core }}}{\partial T}+2 \frac{n_{\text {eff }, \text { Core }}}{\cos \left(\theta_{t}\right)} \frac{\partial \Lambda_{g}}{\partial T}\right] \Delta T . \\
\Delta \lambda_{\text {Clad }, i}=\left[\frac{\Lambda_{g}}{\cos \left(\theta_{t}\right)} \frac{\partial\left(n_{e f f, \text { Core }}+n_{\text {eff }, \text { Clad }, i}\right)}{\partial n_{\text {ext }}}\right] \Delta n_{\text {ext }} \\
+\left[\frac{\Lambda_{g}}{\cos \left(\theta_{t}\right)} \frac{\partial\left(n_{e f f, C o r e}+n_{e f f, C l a d, i}\right)}{\partial T}+\frac{n_{\text {eff }, \text { Core }}+n_{e f f, C l a d, i}}{\cos \left(\theta_{t}\right)} \frac{\partial \Lambda_{g}}{\partial T}\right] \Delta T .
\end{gathered}
$$

For ordinary single mode fibers, the effective index of the core is insensitive to the changes of the external refractive index, therefore (3.2.5) may simplify to:

$$
\Delta \lambda_{B}=0+\left[2 \frac{\Lambda_{g}}{\cos \left(\theta_{t}\right)} \frac{\partial n_{e f f, \text { Core }}}{\partial T}+2 \frac{n_{\text {eff }, \text { Core }}}{\cos \left(\theta_{t}\right)} \frac{\partial \Lambda_{g}}{\partial t}\right] \Delta T
$$

and (3.2.6) may reduce to:

$$
\begin{aligned}
& \Delta \lambda_{\text {Clad }, i}=\left[\frac{\Lambda_{g}}{\cos \left(\theta_{t}\right)} \frac{\partial n_{\text {eff }, \text { Clad }, i}}{\partial n_{\text {ext }}}\right] \Delta n_{\text {ext }} \\
& \quad+\left[\frac{\Lambda_{g}}{\cos \left(\theta_{t}\right)} \frac{\partial\left(n_{e f f, \text { Core }}+n_{e f f, \text { Clad }, i}\right)}{\partial T}+\frac{n_{\text {eff }, \text { Core }}+n_{\text {eff }, \text { Clad }, i}}{\cos \left(\theta_{t}\right)} \frac{\partial \Lambda_{g}}{\partial T}\right] \Delta T .
\end{aligned}
$$

Equation (3.2.8) shows that cladding resonances are sensitive to external refractive index change and their sensitivity is not the same for all of the modes. Furthermore, the temperature induced shifts for $\Delta \lambda_{B}$ and $\Delta \lambda_{C l a d, i}$ are seen to be very similar since $\frac{\partial\left(n_{\text {eff } f \text { Core }}+n_{\text {eff }, \text { Clad }, i}\right)}{\partial T} \approx \frac{2 \partial\left(n_{\text {eff }, \text { Core }}\right)}{\partial T}$ and $2 n_{\text {eff }} \frac{\partial \Lambda}{\partial T} \approx\left(n_{\text {eff,Core }}+n_{\text {eff }, \text { Clad }, i}\right) \frac{\partial \Lambda}{\partial T} \approx 0$ due to the low CTE of silica. 

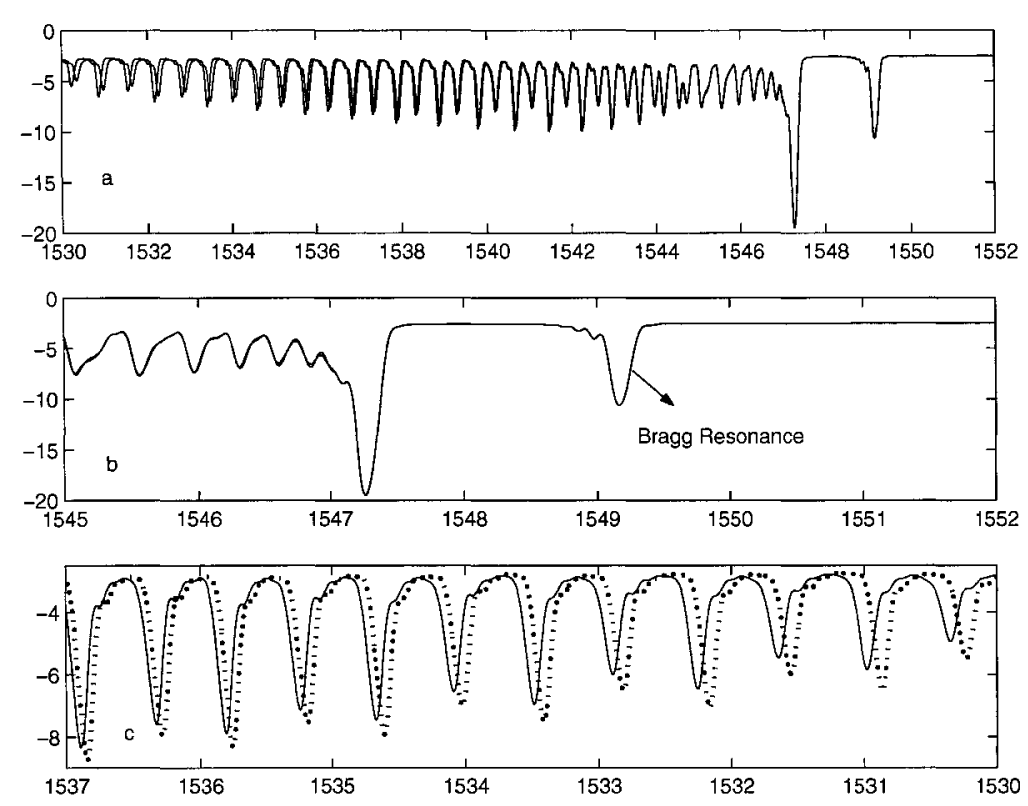

Figure 3.5: a: Overlap of the transmission spectra of a TFBG with two different external refractive indices, $b$ : The Bragg resonances overlap in two cases because the temperature is fixed and the core mode is not sensitive to the external refractive index change, c: The cladding modes are sensitive to the external refractive index change. the solid line represents the spectrum when $n_{\text {ext }}=1.4$ and the dashed line corresponds to the case of $n_{\text {ext }}=1.42$

Another mechanism that acts similar to TFBGs is Long Period Gratings(LPG). LPGs also couple the light from core mode to cladding modes but with a different mechanism than TFBGs. TFBG in comparison with LPG are more compact and less sensitive to bending and couple simultaneously to tens of cladding modes within a measuring window smaller than $20 \mathrm{~nm}$.

\subsection{Fabrication}

\section{Photosensitivity}

Fiber photosensitivity was first observed in an experiment performed by Hill et al[20] at the Communications Research Center of Canada in 1978. This phenomenon causes 


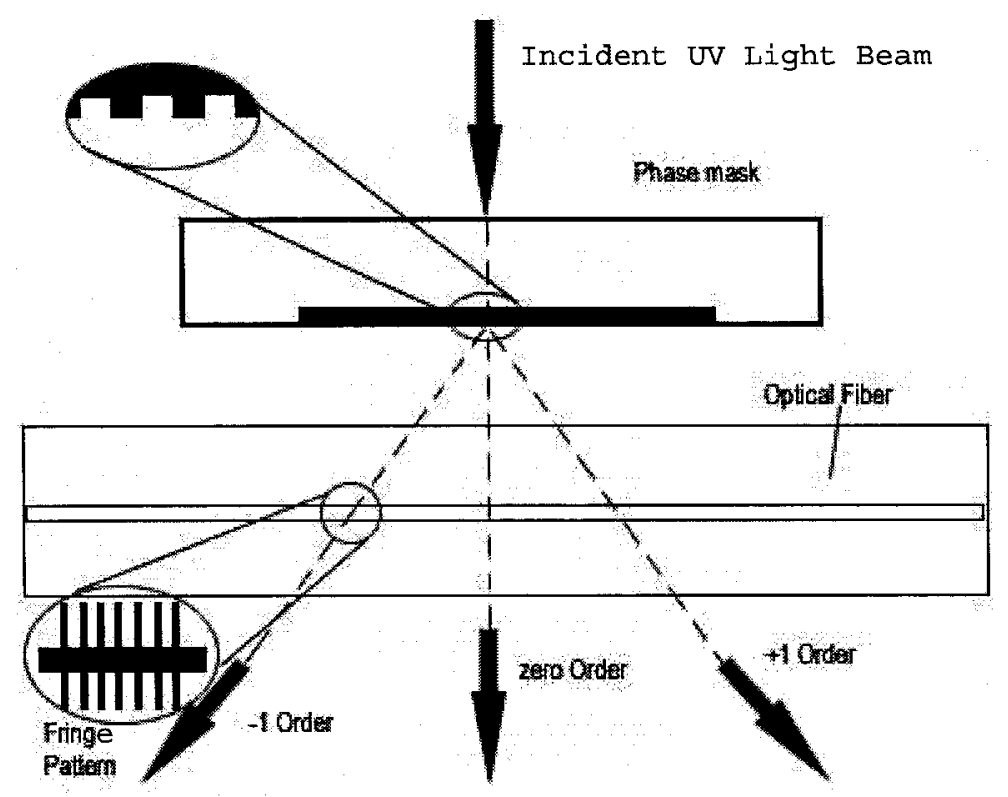

Figure 3.6: Phase-mask technique setup to write a FBG (from "Fiber Bragg Grating Technology, Fundamentals and Overview" By K. O. Hill and G. Meltz, J. Lightwave Technol. vol 15, no 8, pp 1263-1276, August 1997.)

the refractive index of the core of Ge-doped silica fiber exposed to laser radiation to change. This change is permanent and can be used to photo-induce gratings in optical fiber. Since the discovery of this phenomenon there have been several techniques developed to print grating planes in optical fiber, each having its own merits and drawbacks. Of these techniques one can mention dual-beam holographic technique in which the interference pattern of a light with itself is used to print the grating planes[21]. The main advantage of this techniques is that a wide range of grating periods, $\Lambda$, is obtained very easy.

Although dual-beam techniques exhibits good performance, commercial production of FBG makes use of phase-mask techniques. Phases-masks are usually fabricated on quartz or silica substrates. The basic idea relies on using a phase mask with a periodicity related to the desired grating period. Figure 3.6 shows the setup used to print a FBG in the core of a single mode fiber. The main advantage of the phase 
mask method is that, due to non-interferometric nature of the technique, there is no need for a laser source with high spacial coherence, even an UV lamp can be used to write the grating. Most importantly, the phase mask technique allows the mass production of identical gratings. Phase mask technique also allows the fabrication of FBGs with variable periods(chirped FBG). In this method the quality of the grating depends completely on the quality of the phase mask and any imperfections in the phase mask is regenerated precisely in the grating.

TFBGs are also fabricated using phase-mask. The setup is illustrated in Figure 3.7. In order to write TFBGs people employ different techniques, some keep the phase mask and incident beam fixed and rotate the fiber while others keep the fiber constant and rotate the phase mask. This setup is the setup that we employ in our lab to fabricate TFBG. the angel $\alpha$ gives an indication of the actual grating tilt angle $\theta_{t}$

\subsection{Conclusion}

Fiber Bragg grating as a fundamental building block of optical sensors was introduced. The dependence of the central Bragg wavelength on temperature and strain was shown mathematically. Coupled mode theory was employed to derive the mathematical relationship of the FBG reflectivity and it was mentioned that reflectivities as high as $99 \%$ are achievable in practice.

By tilting the grating planes of the FBG with respect to the fiber longitudinal axis, a new class of grating with interesting properties was introduced. It was understood that this tilt angel causes the light, that otherwise was guided in the core, to be coupled into several cladding modes; therefore in the transmission spectrum one can observe several cladding resonances (stop bands) in addition to the usual Bragg resonance. If this tilt angle is small, these grating are called weakly tilted fiber Bragg 


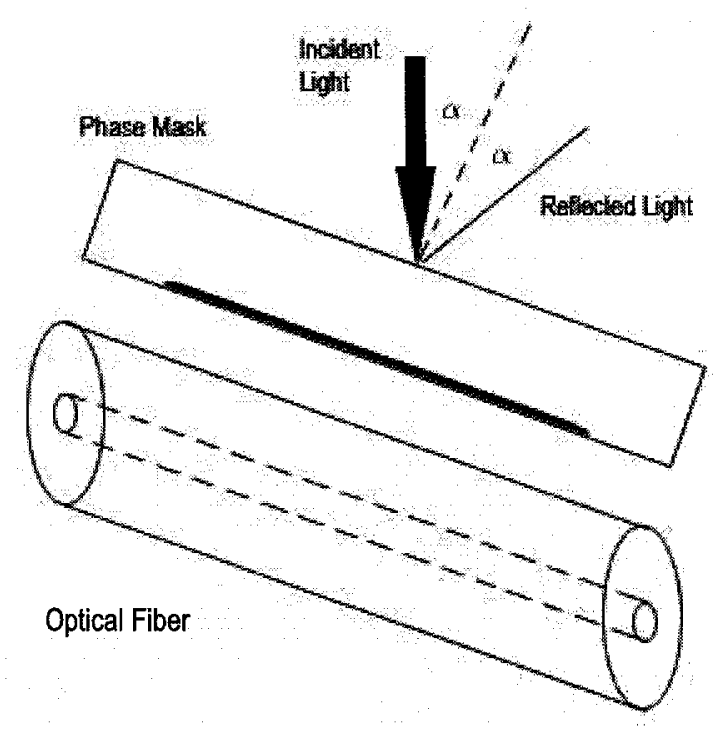

Figure 3.7: Phase-mask technique setup to write a TFBG

gratings and are suitable to measure the temperature and the refractive index of the surrounding environment simultaneously. Through mathematical manipulation, it was revealed that the core resonance center wavelength (Bragg wavelength) is only sensitive to temperature and strain while the cladding resonances, because of traveling between the cladding and the surrounding material interface, can sense the change of the external refractive index and have approximately the same temperature and strain dependence as the Bragg resonance. This last feature allows the measurement of the SRI from the relative shift in the cladding mode position relative to the Bragg wavelength. Fabrication techniques including the most important one, phase-mask, were addressed and different setups to achieve ordinary fiber Bragg gratings or tilted ones were illustrated. 
"The eye sees only what the mind is prepared to comprehend"

- Henry L Bergson

\section{Chapter 4}

\section{AWG as Sensor Interrogator, Simulation and Results}

In this chapter, the requirements for an AWG to be employed as a sensor interrogator are reviewed briefly and several design examples are presented afterwards. The advantages and disadvantages of each design are discussed and the proper design for fabrication is proposed as the conclusion.

\subsection{Requirements}

As mentioned in Chapter 2, a sensor interrogator in order to be utilizable in field applications should exhibit several characteristics including portability, low cost, high measurement accuracy, high measuring speed, high resolution, large measurement range and capability of multiplexing. Using the AWG as sensor interrogator enables not only tracking the shifts in the wavelength but also tracking the changes in the amplitude of the signal coming from the sensor, which in some applications is a key feature. The characteristics of the AWG are determined by application, for example, if the application is to interrogate a single fiber Brag grating, then it is not really important what the channel spacing of the AWG is whereas the bandwidth in this application may be important. In contrary, if the task is to interrogate a TFBG, then the channel spacing also matters. In designing a sensor interrogator, size is another 


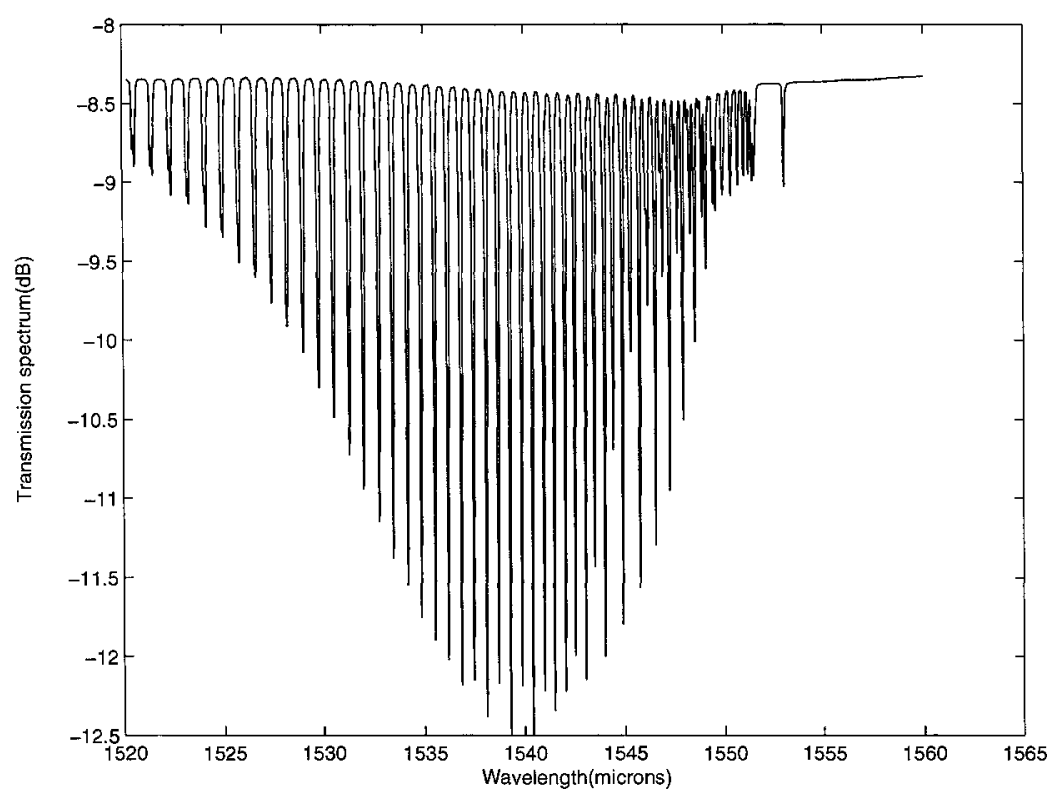

Figure 4.1: Experimental transmission spectrum of a TFBG with a tilt angle of 6 degrees.

issue which is very important and it should be kept as small as possible. Keeping the size small results in increasing crosstalk (there is a trade-off between the size of the device and crosstalk, as will be discussed later).

\subsection{Design and Simulation}

Figure 4.1 depicts the transmission spectrum (obtained by experiment) of a TFBG with a tilt angle of 6 degrees. This grating has been used as a refractive index sensor during this work. Designing an AWG to interrogate a spectrum such as Figure 4.1 requires exploring the spectrum first. Examining the spectrum depicted in Figures 4.2 and 4.3 reveals that the spacing between the resonances is slightly different in two regions of the spectrum.

In the vicinity of the Bragg resonance, around the wavelength of $1553 \mathrm{~nm}$, the resonances occur with a spacing of around $0.4 \mathrm{~nm}$, whereas far from the Bragg resonance, 


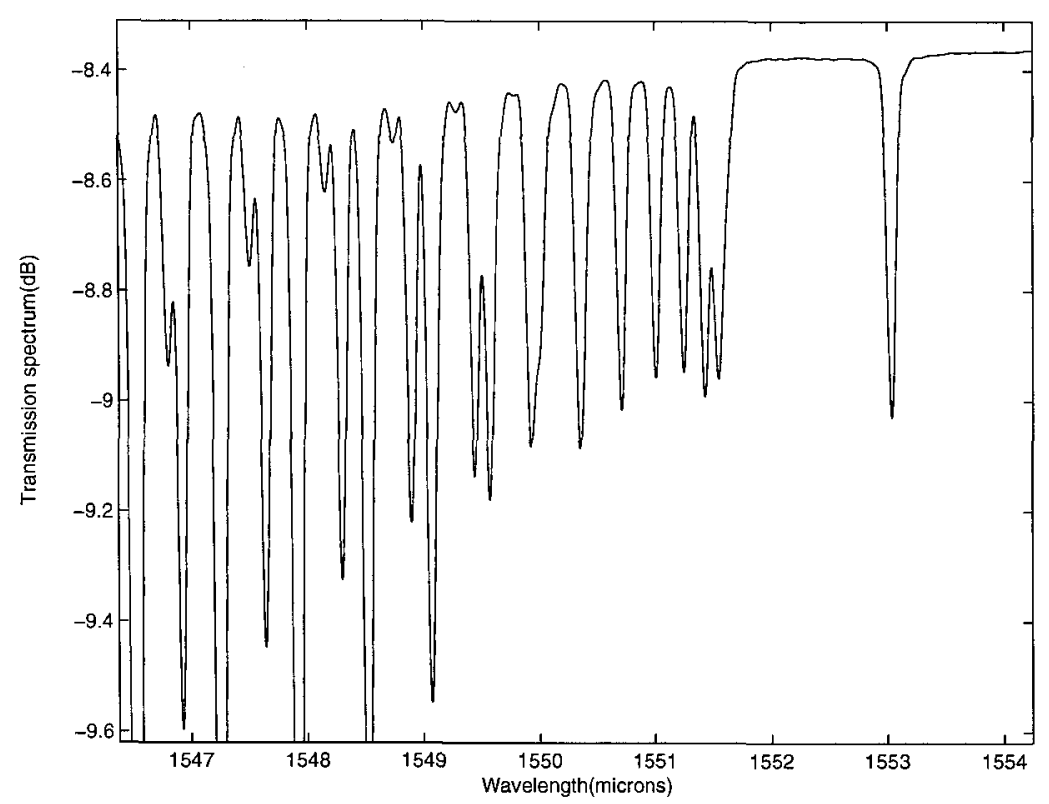

Figure 4.2: Spectrum of a TFBG with a tilt angle of 6 degrees, in the vicinity of the Bragg resonance.

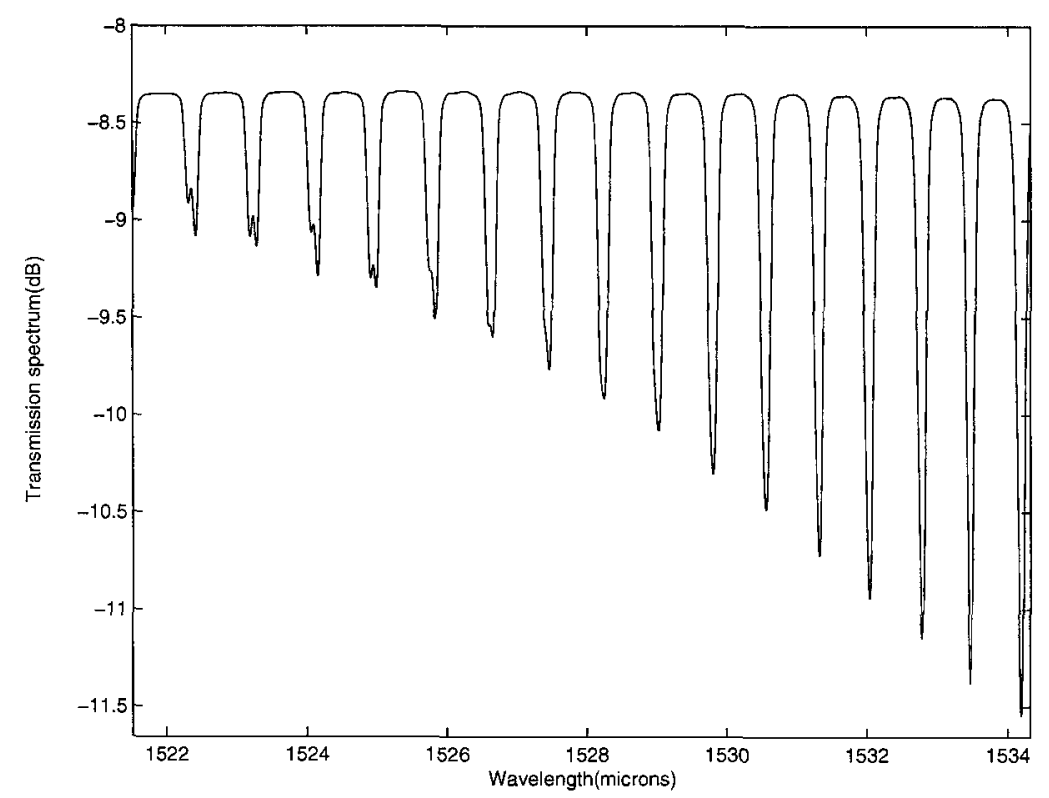

Figure 4.3: Spectrum of a TFBG with a tilt angle of 6 degrees, far from the Bragg resonance 
the spacing between resonances increases to $0.6 \mathrm{~nm}$. Based on this information, designing the AWG with a channel spacing of $0.5 \mathrm{~nm}$ seems reasonable. The bandwidth of the resonances is also around $0.3 \mathrm{~nm}$, by keeping in mind this point that here we try to track the shift in the resonance's location due to the change of external refractive index, one may deduce that the narrower the bandwidth, the better performance for this task; therefor we try to design AWG with a bandwidth as narrow as possible. This will result in a more accurate resonance shift tracking.

The pass-band shape of the AWG response is desired to be Gaussian. The reason for this is that designing a flattened response AWG is in principle harder than designing a Gaussian shape pass-band. Apart from being harder in design, it is not necessary indeed to design a flattened response device in sensor applications where the goal is to keep the interrogator unit (AWG here) as simple and as small as possible and thus as low cost as possible, Furthermore, a Gaussian shape pass-band will react more to wavelength offset.

The range of wavelengths over which the AWG should operate is determined again by having a look over the spectrum of TFBG. In order to be able to cover the whole spectrum, the AWG should be able to cover a wavelength range of about $32 \mathrm{~nm}$, from $1521 \mathrm{~nm}$ to $1553 \mathrm{~nm}$. With the aforementioned channel spacing of $0.5 \mathrm{~nm}$, the number of channels will be at least 64 .

At this point now we can summarize the design procedure as the following steps:

- Output Waveguide Spacing $d_{o}$ : In order to obtain low crosstalk levels, this number should be chosen as large as possible. A reasonable number which gives a good crosstalk is $20 \mu \mathrm{m}$.

- Array Waveguide Spacing $d_{a}$ : The smallest value that fabrication technology allows is chosen. The number that we pick up is $9 \mu \mathrm{m}$.

- Channel Spacing $\Delta v_{c}$ or $\Delta \lambda$ : As discussed, channel spacing is set to be equal 


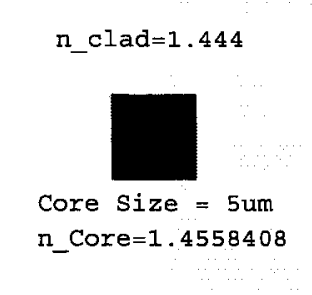

Figure 4.4: Cross section of the waveguide used in this work

to $0.5 \mathrm{~nm}$.

- Free Spectral Range $\Delta v_{F S R}$ : Once number of channels and channel spacing are determined, FSR is actually determined automatically because in practice $F S R=N_{c h} . \Delta \lambda=65 \times 0.5=32.5 \mathrm{~nm}$.

- Grating Order $m$ : is determined as follow as follow:

$\mathrm{m} \leq \frac{\lambda_{c}}{F S R}=\frac{1539}{32.5} \approx 47$.

where $\lambda_{c}$ is the central wavelength and FSR is free spectral range. In here $\mathrm{m}$ is chosen to be 47 .

- Length of the Slab Waveguide $L_{s}$ : Length of the free coupler region is calculated below:

$L_{s}=\frac{N_{s} d_{o} d_{a}}{m \Delta \lambda}=\frac{1.4558408 \times 20 \times 9}{47 \times 0.0005}=11151.12102 \mu \mathrm{m}$.

where $N_{s}$ is the refractive index of the slab waveguide and $\Delta \lambda$ is the channel 


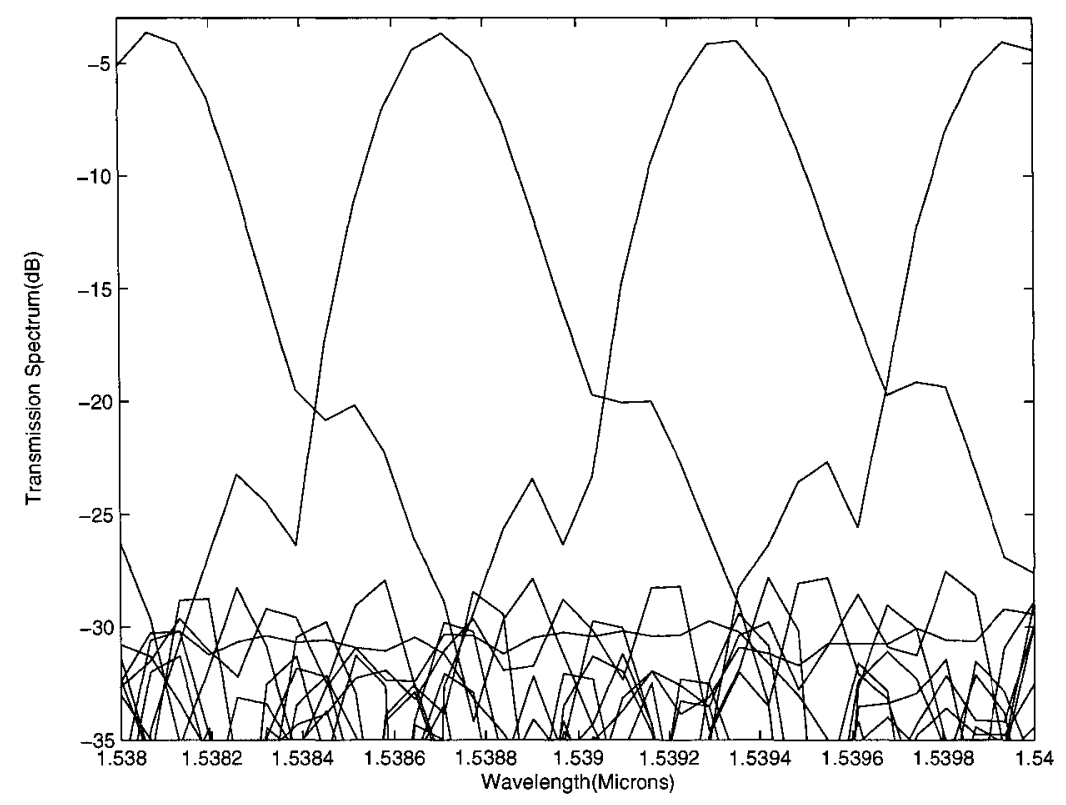

Figure 4.5: Transmission Spectrum of the $A W G$

spacing.

- Number of Waveguides in Array $N$ : Is set to be 181 .

- The length increment $\Delta L$ : is obtained through

$\Delta \mathrm{E}=\frac{m \lambda_{c}}{N_{g}}=\frac{47 \times 1.539}{1.4543699}=49.7349402 \mu \mathrm{m}$.

where $N_{g}$ is the waveguide effective index in the array and input/output section.

- Half Angle Aperture Opening $\theta_{a}$ :

$\theta_{a}=\frac{(N-1) d_{a}}{2 L_{s}}=\frac{180 \times 20}{2 \times 11151.12} \approx 9$.

where $\mathrm{N}$ is the number of waveguides in the array.

This device has been simulated by the use of Optiwave software, OptiBPM 6.1. Figure 4.5 shows the transmission spectrum of this device. 


\begin{tabular}{|l|c|c|c|c|c|}
\hline Design Parameter & Symbol & Design 1 & Design 2 & Design 3 & Unit \\
\hline \hline Center Wavelength & $\lambda_{c}$ & 1539 & 1539 & 1539 & $\mu \mathrm{m}$ \\
\hline Output Waveguide Separation & $d_{o}$ & 20 & 9 & 25 & $\mu \mathrm{m}$ \\
\hline Array Waveguide Separation & $d_{a}$ & 10 & 9 & 9 & $\mu \mathrm{m}$ \\
\hline Number of Arrayed Waveguides & $\mathrm{N}$ & 181 & 181 & 181 & \\
\hline Channel Spacing & $\Delta \lambda$ & 0.5 & 0.5 & 0.5 & $\mathrm{~nm}$ \\
\hline Grating Order & $\mathrm{m}$ & 45 & 47 & 47 & \\
\hline Number of Channels & $N_{c h}$ & 67 & 65 & 65 & \\
\hline Coupler Length & $L_{s}$ & 12940.81 & 5018 & 11151.12 & $\mu \mathrm{m}$ \\
\hline Size of the Wafer & $* * *$ & $40 \times 50$ & $21 \times 22.8$ & $19 \times 23$ & $m \mathrm{~m}^{2}$ \\
\hline Slab Index & $N_{s}$ & 1.4558408 & 1.4558408 & 1.4558408 & \\
\hline Waveguide Effective Index & $N_{g}$ & 1.4543699 & 1.4543699 & 1.4543699 & \\
\hline Receiver Waveguide Spacing & $* * *$ & 250 & 250 & 50 & $\mu \mathrm{m}$ \\
\hline Adjacent Channel Crosstalk & $\chi_{\text {adj }}$ & -23 & -15 & -24 & $\mathrm{~dB}$ \\
\hline Non-adjacent Channel Crosstalk & $\chi_{\text {nad }}$ & -31 & -21 & -35 & $\mathrm{~dB}$ \\
\hline
\end{tabular}

Table 4.1: Comparison between 3 different $A W G$ designs

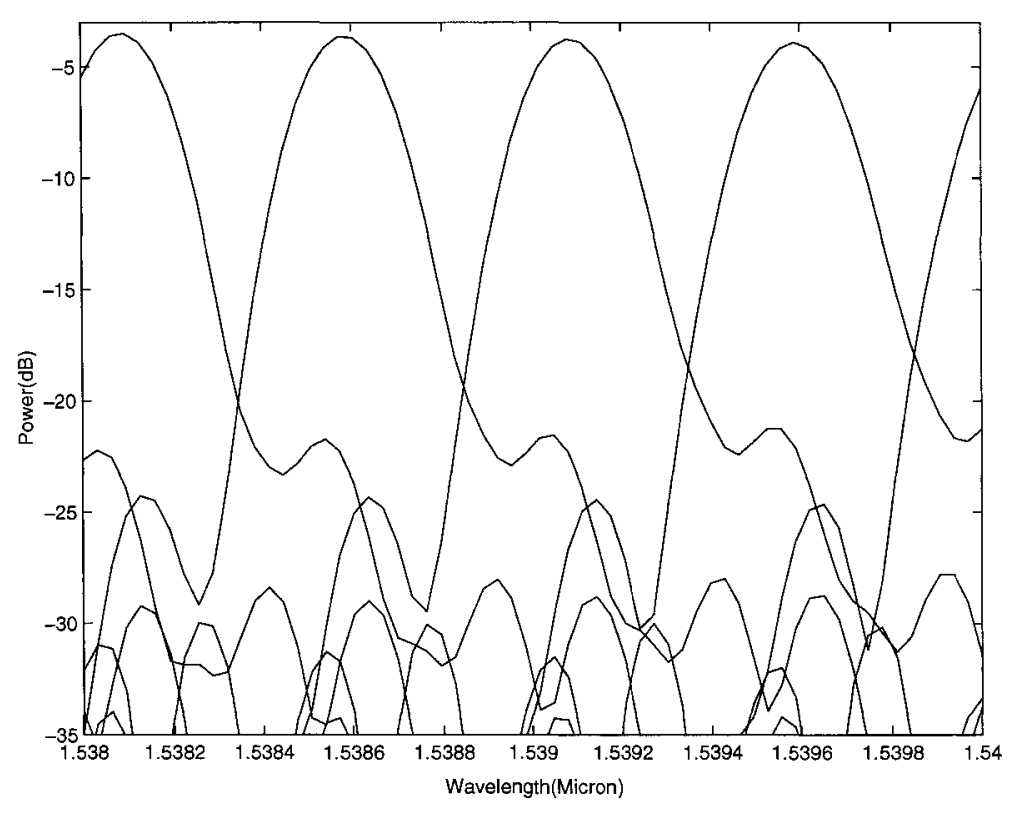

Figure 4.6: Transmission Spectrum of design 1 


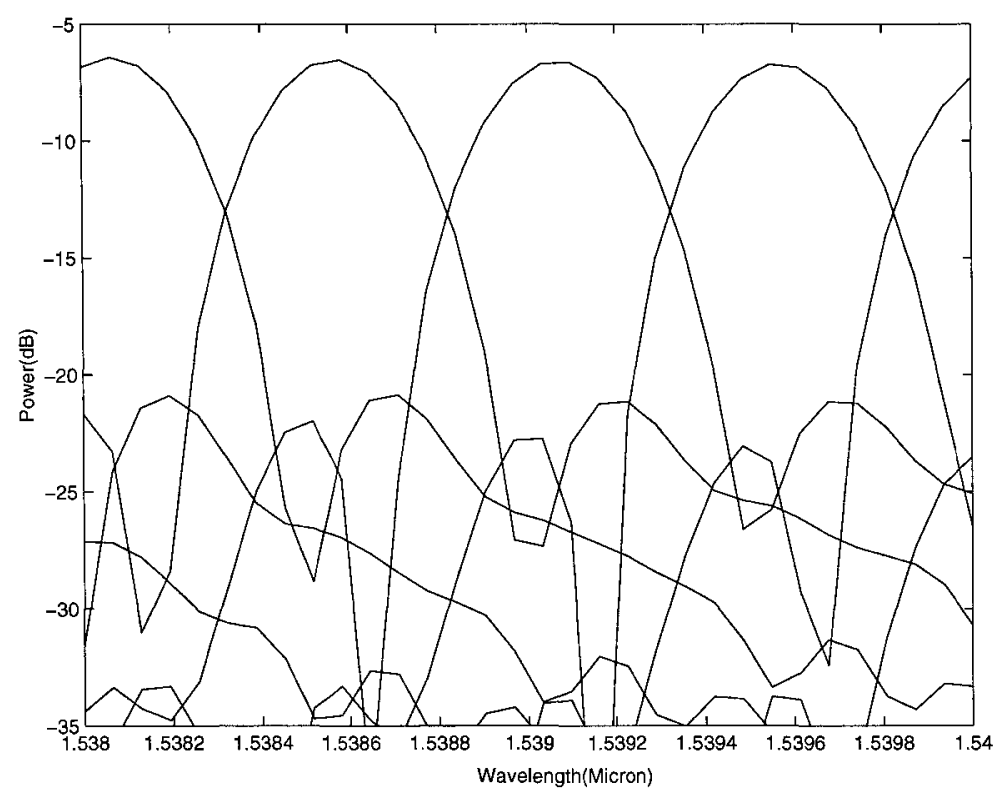

Figure 4.7: Transmission Spectrum of design 2

\subsection{Results}

In order to be able to find out the optimum AWG design to perform the task of interrogation of TFBG spectrum, several simulations have been performed. Table 4.2 summarizes the design parameters of these different designs. The optimum design is the one which with the smallest size, exhibits the best performance. Figures 4.6 and 4.7 illustrate respectively the spectrums of AWG designs number 1 and 2 whereas Figure 4.8 depicts the spectrum of design number 3. Comparing the three different designs reveals that design 3 exhibits the best performance while having the smallest size. In designs number 1 and 2 the spacing between receiver waveguides is $250 \mu \mathrm{m}$. This particular value is chosen in order to make the design compatible with fiber arrays $^{1}$ ( in which the spacing between optical fibers is usually $127 \mu \mathrm{m}$ or $250 \mu \mathrm{m}$ ). In design number 3 one observes that the spacing between receiver waveguides has been decreased to $50 \mu \mathrm{m}$ which is a huge change in comparison with designs number

\footnotetext{
${ }^{1} \mathrm{~V}$ Groove for example
} 


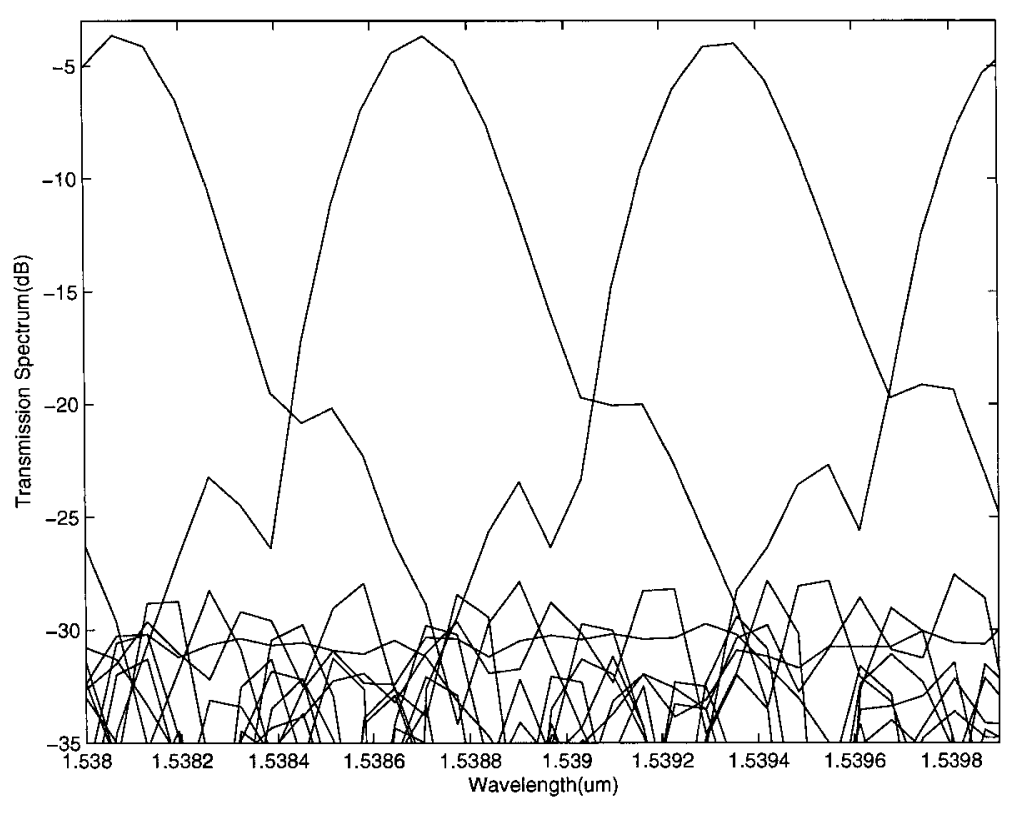

Figure 4.8: Transmission Spectrum of the design 3

1 and 2. The reason of this change is that $\mathrm{CPFC}^{2}$ that was in charge of fabricating the AWG imposes a limitation on the size of the device ( the design has to be able to fit in a circle of $31 \mathrm{~mm}$ diameter), therefore we had to sacrifice the convenience of having a $250 \mu \mathrm{m}$ spacing between the receiver waveguides to achieve the goal of fitting the device in the required dimensions and keeping the number of channels equal to 65. Apart from this, the ultimate goal of this project is to couple the AWG directly to the detector array, in which the spacing between the elements is usually $25 \mu \mathrm{m}$ or $50 \mu \mathrm{m}$; Thus choosing a $50 \mu \mathrm{m}$ spacing between the receiver waveguides was not that much irrelevant. Design number 3 presents a cross talk level as low as $-24 \mathrm{~dB}$ from adjacent channel and $-35 \mathrm{~dB}$ from non-adjacent channel. In designing an AWG for sensor interrogator application, crosstalk is very tolerable. Crosstalk levels as low as $-15 \mathrm{~dB}$ from adjacent channels are gladly acceptable because in sensor applications it is the shift in the position of the center wavelength which is of interest and not

\footnotetext{
${ }^{2}$ Canadian Photonic Fabrication Center
} 


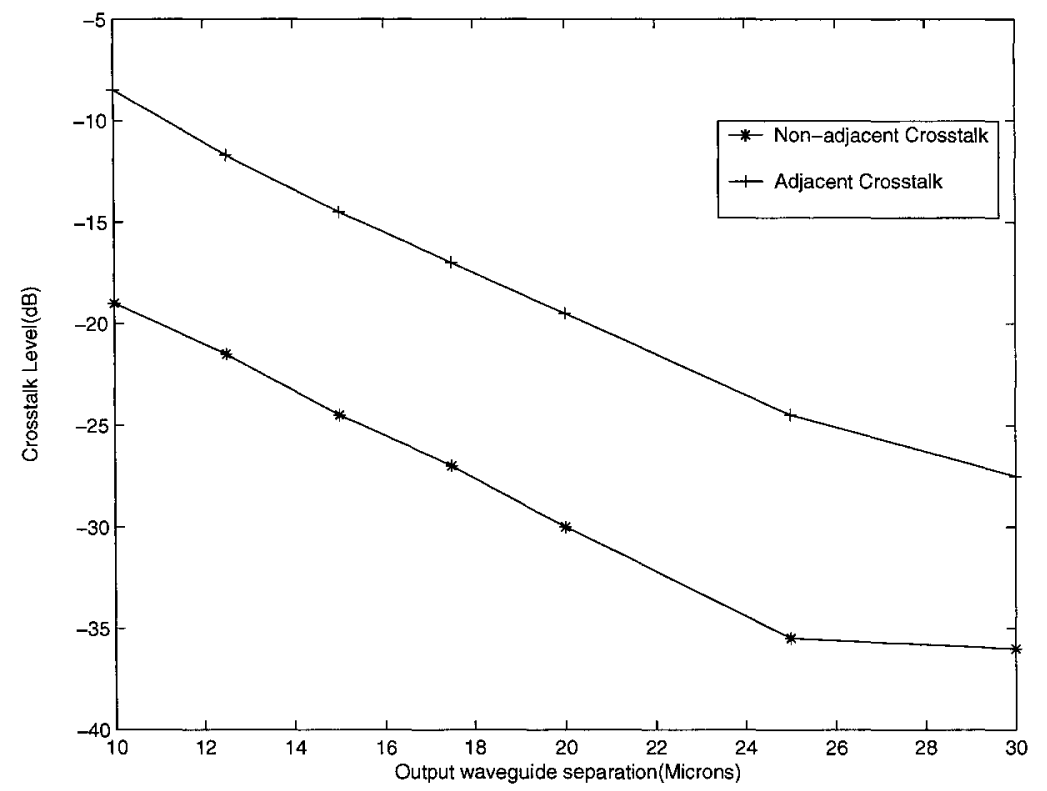

Figure 4.9: Relationship between crosstalk and output waveguide spacing

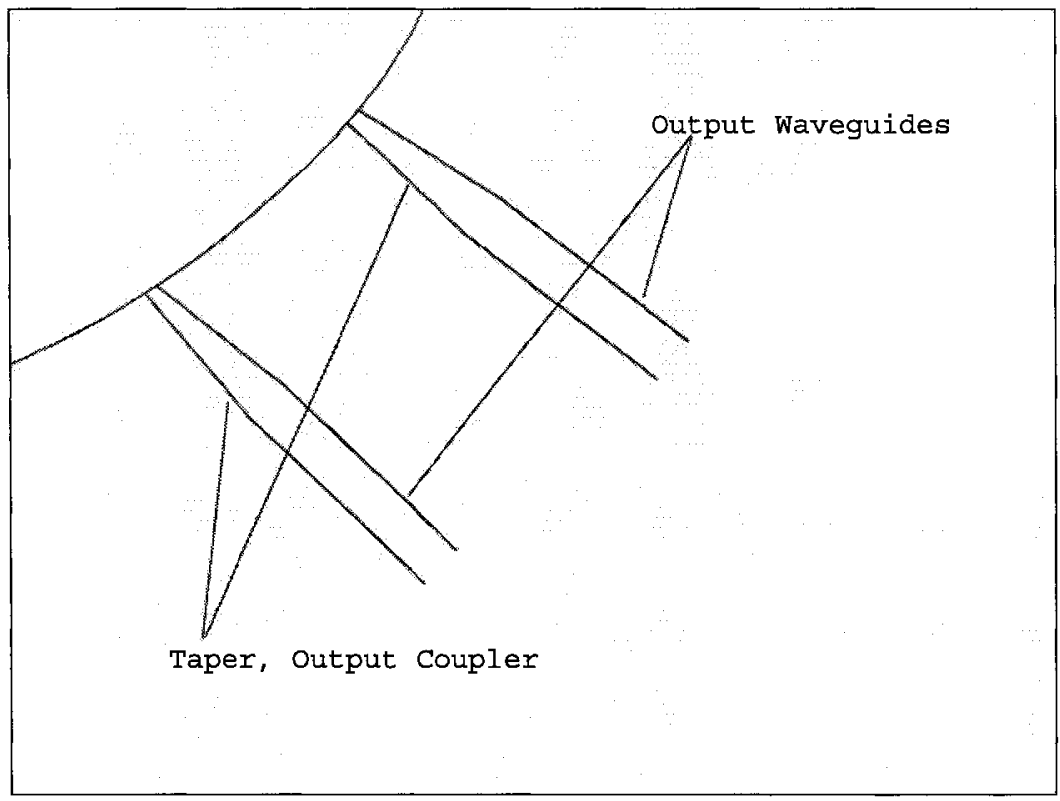

Figure 4.10: Type of taper used in output coupler section 


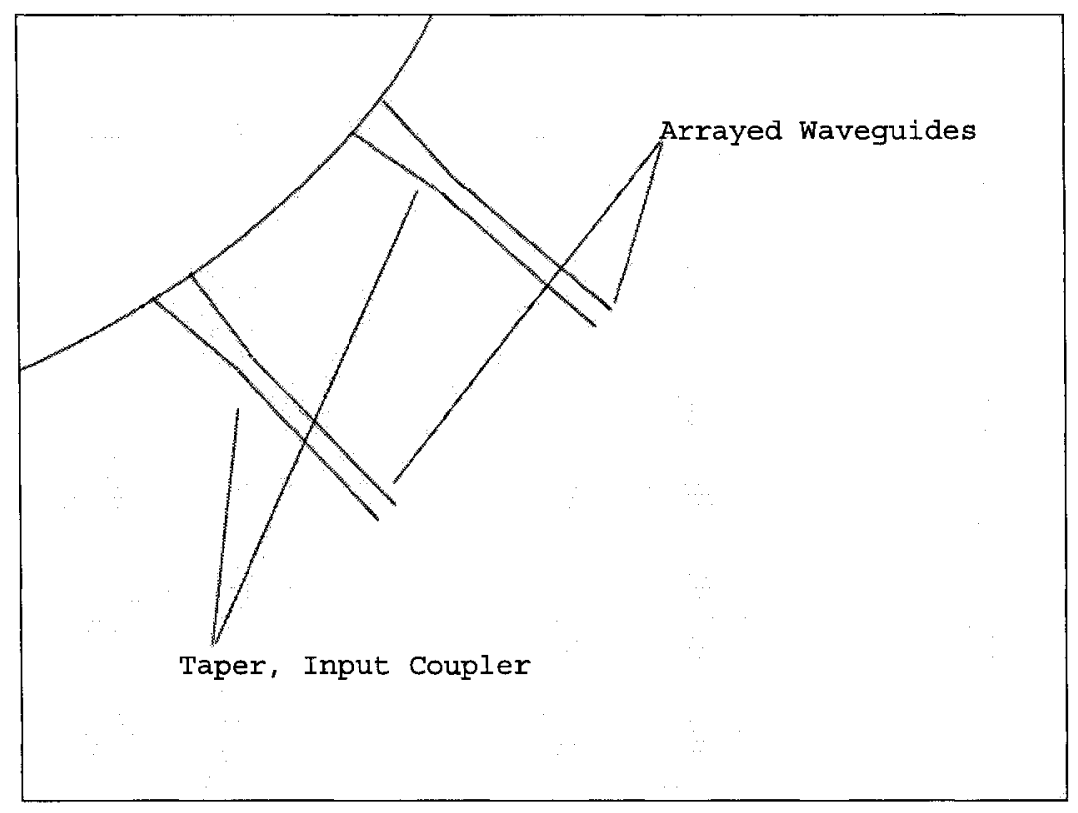

Figure 4.11: Type of taper used in arrayed waveguides section

the signal itself, while in telecommunication the signal itself is important and should be noise free as much as possible and thus cross talk is more critical. Designing an AWG with very low crosstalk levels, make the interrogator unit either big in size or expensive in price which is not acceptable for sensor applications. In an AWG device, the main factor that effects the crosstalk is the output waveguide separation. This parameter also directly affects the coupler length. The larger the value of output waveguide separation, the lower the crosstalk, the bigger the device. Figure 4.9 clearly shows the inverse relationship between output waveguide spacing and crosstalk (this relationship also depends on the waveguide $\mathrm{V}$ number, these graphs are plotted for waveguides used in this work).

Another way that slightly improves the crosstalk is employing tapers in the junction of the waveguides and slabs both in input/output section and in the array section (compare Figure 4.8 and Figure 4.12). The type of tapers used in each section is different. Figure 4.10 shows the type of taper which is used in output coupler section. 


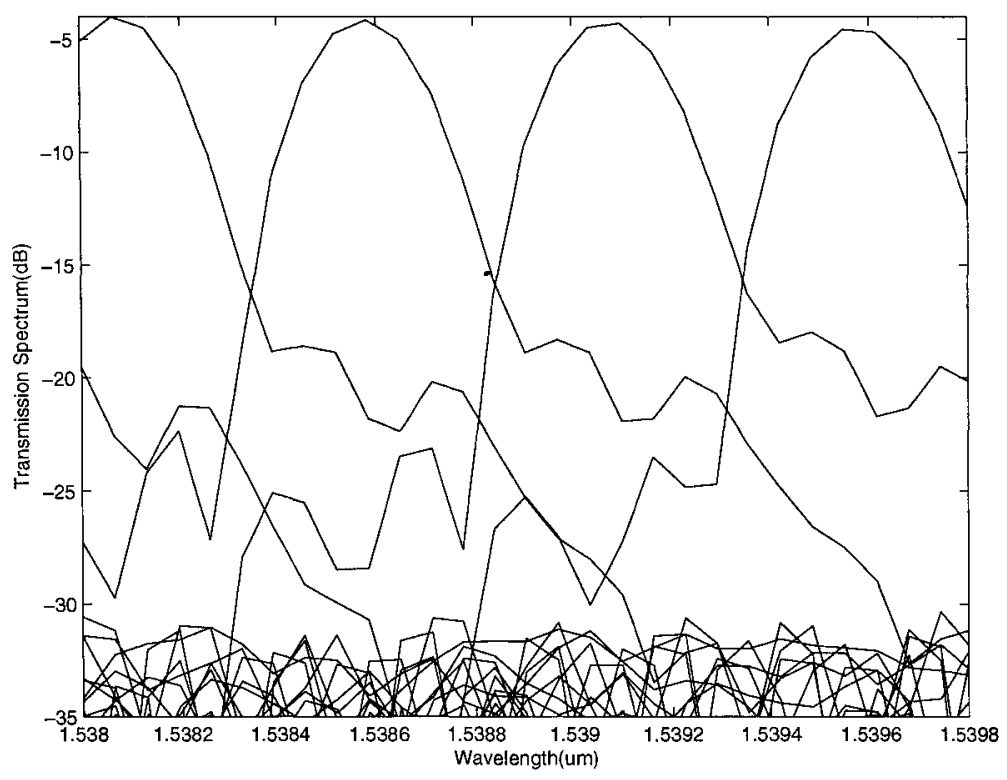

Figure 4.12: Transmission Spectrum of the design 3 with tapers

Using this kind of taper results in blocking some of the undesired power from other channels present in this place and thus improving both adjacent and non-adjacent crosstalk. Employing this kind of taper is specially useful in improving non-adjacent crosstalk. Figure 4.11 illustrates the type of tapers used in the array section. Utilizing this kind of taper results in collecting more power at the end of input coupler and thus improving crosstalk. Figure 4.12 shows the effect of tapers on the spectrum of the AWG.

Bandwidth is another characteristics of AWG which is inversely related to output waveguide spacing. The larger the output waveguide spacing, the narrower the bandwidth (Figure 4.13). This inverse relationship is desirable to us since we are after a narrow band AWG to be able to track the shifts in the resonances of the TFBG spectrum. All of the considerations lead to design number 3 with tapers as the optimum design. 


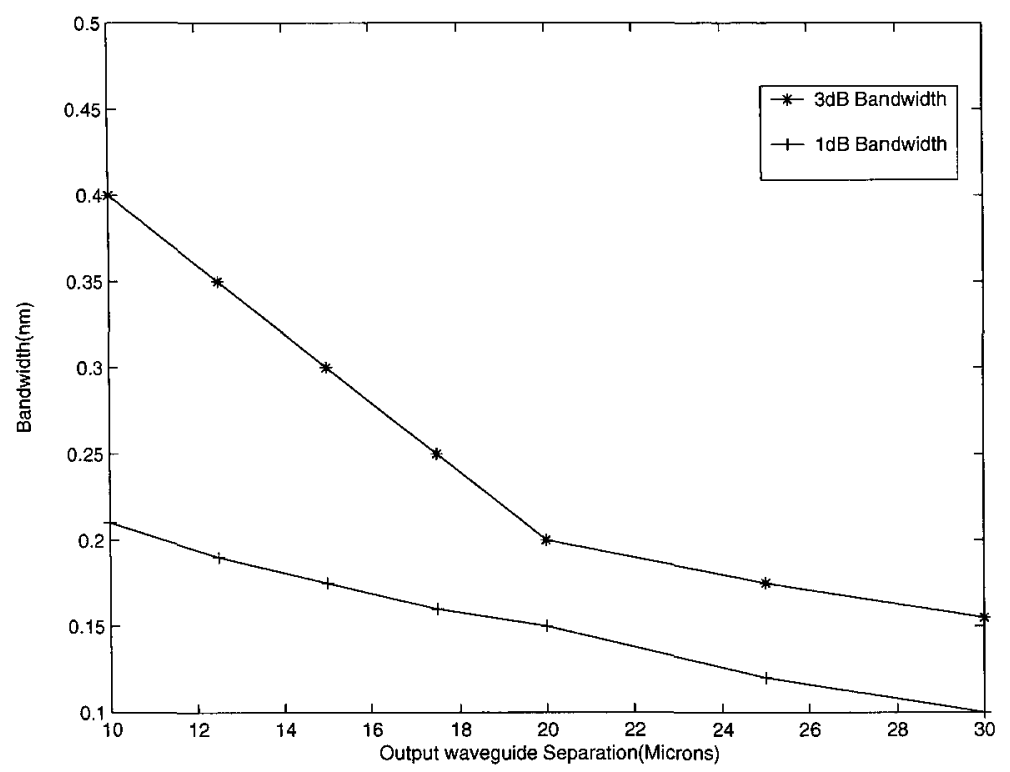

Figure 4.13: Relationship between bandwidth and output waveguide spacing

\subsection{Conclusion}

Arrayed waveguide gratings are promising devices to be employed as sensor interrogator. They can satisfy several requirements of an ideal interrogator such as small size and low cost. AWGs can be customized in order to fulfill the requirements of any special application. The AWG presented in this chapter as design 3 is a suitable design to be employed as the spectrum analyzer of a TFBG spectrum. This design exhibits a very good crosstalk level for sensor applications and also benefits from a very small size. The design is very simple and avoids any complexity whatsoever. A full description of this design is presented in Appendix A. 
"On ne voit qu'avec le cour, l'essentiel est invisible pour les yeux."1

- Antoine de Saint Exupery

\section{Chapter 5}

\section{TFBG as a Refractive Index Sensor, Experiment, Simulation and Results}

In this Chapter, firstly, the capability of a TFBG, as a sensor which can sense the index of refraction in surrounding material (SRI), will be reviewed briefly. Then, the experiment conducted to employ this kind of grating as a real sensor is presented. The numerical simulation performed during this project as a tool to verify the experimental results, will be stated. Finally, the results will be discussed to give a clear understanding of the issue. At the end of this Chapter, a very simple piece of work done to interrogate the TFBG with an AWG is presented.

\subsection{Concept Review}

In Chapter 3, the TFBG as a type of sensor which is capable of sensing the refractive index in surrounding environment, was discussed. It was showed there that this capability of sensing the change in surrounding material refractive index comes from the fact that tilted grating planes couple the light into cladding modes, which are guided along cladding and any change in external refractive index changes their propagation constant and thus changes the exact position on the wavelength axis where they

\footnotetext{
${ }^{1}$ It is only with the heart that one can see rightly and not the eyes.
} 


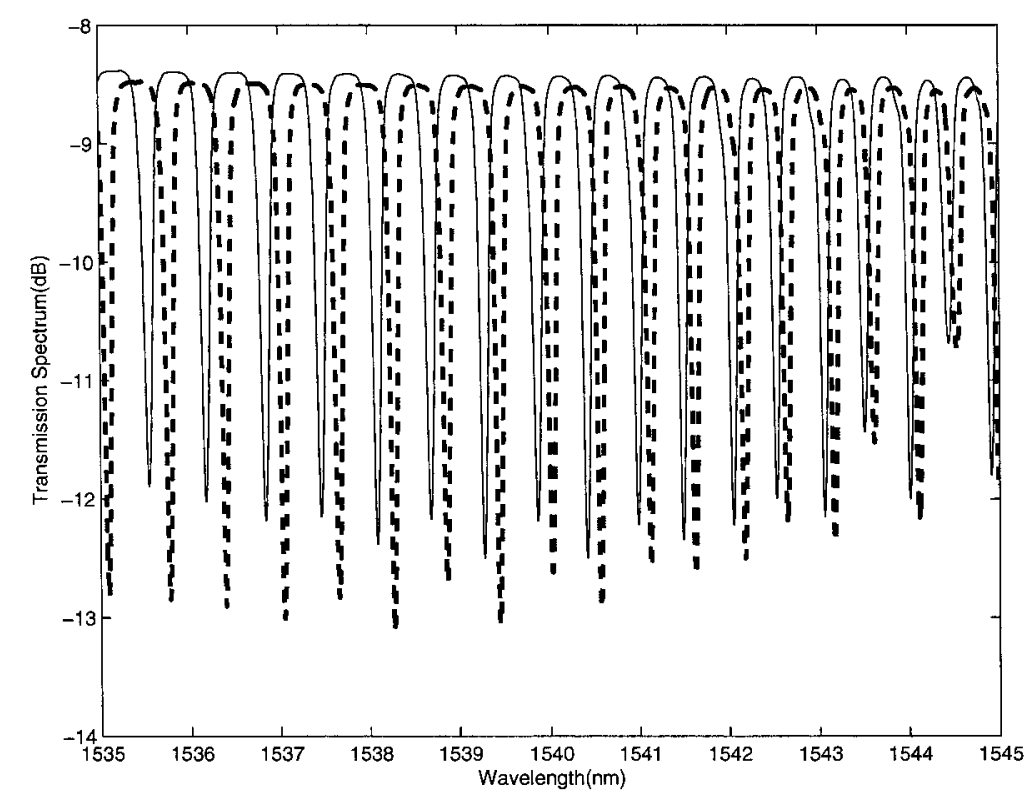

Figure 5.1: Overlap of the spectrums of a TFBG in the air (solid line) and in a material with a refractive index different from air (dashed line).

resonate. Tracking these shifts in the resonance positions, provides one with either the absolute value of the external environment refractive index or the change in the refractive index. Figure 5.1 illustrates the idea. One should keep in mind this point that the sensitivity of different modes to a change in the refractive index of the surrounding material is different and thus different modes will experience different shifts due to a change in SRI.

\subsection{Experiment Setup}

The basic idea in this experiment is to measure the transmission spectrum of a TFBG in different SRIs. A TFBG is inscribed on a standard single mode fiber. The tilt angle of the grating is 6 degrees. The fiber is connected to JDS swept wavelength system which is employed to measure and record the transmission spectrum of the grating. All the readings are done at room temperature which was $23^{\circ} \mathrm{C}$ (constant 


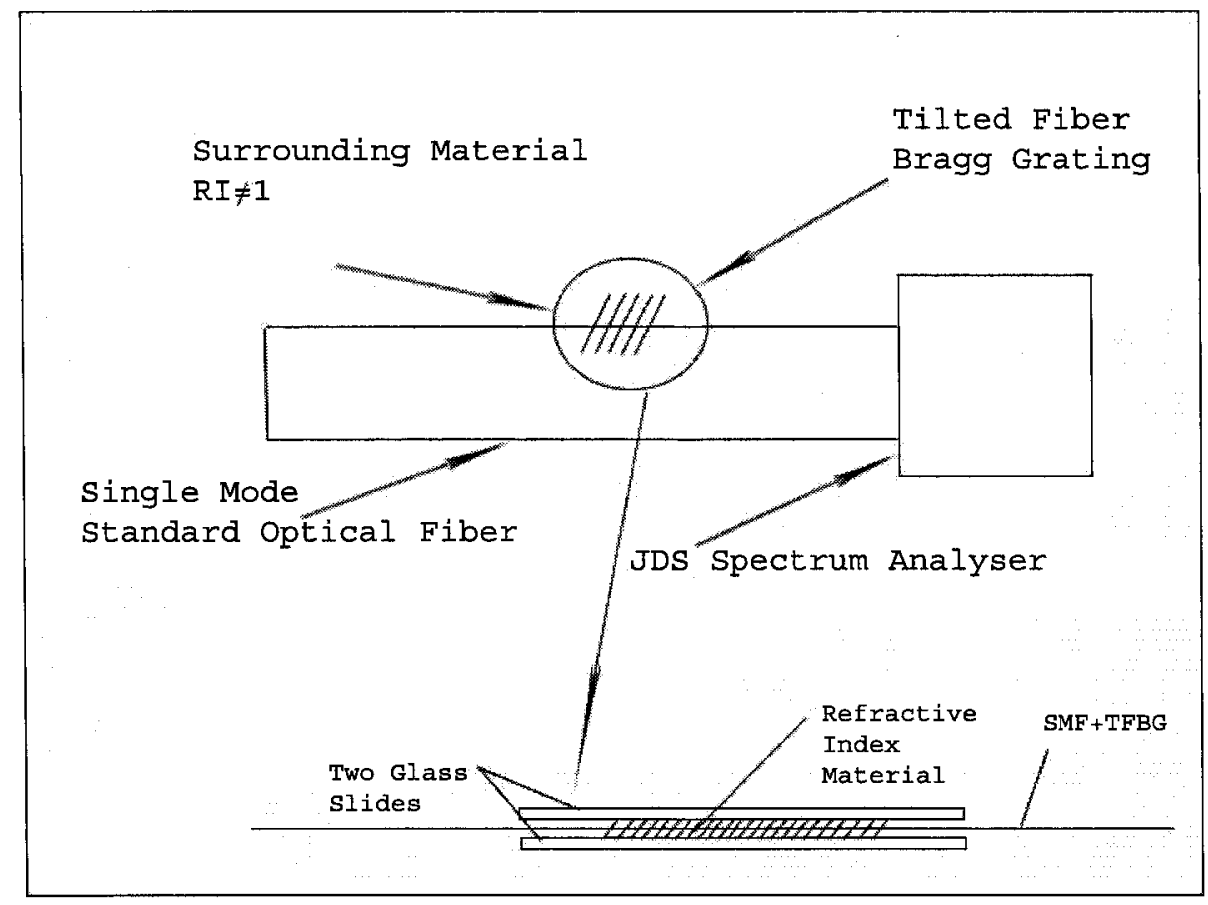

Figure 5.2: Schematic diagram of the experiment Setup).

temperature). In order to keep the strain constant as well, two glass slides were employed. The first slide was taped on the work table, then that part of the fiber that contained the grating, was taped on it. The solution which was used as the surrounding refractive index liquid was trickled by the use of a pipet on the grating, then the second slide was placed gently on the first slide. In this way, the strain on the fiber was to a great extent set to be the same on the whole process of experiment. However, any changes in the strain or temperature can be corrected in data processing time. Change in the temperature or strain on the fiber will cause all the resonances to displace similarly (as seen in Chapter 3). Therefore, by subtracting the Bragg wavelength shift from all the cladding mode shifts or equivalently by measuring the distance between the Bragg resonance and each cladding mode resonance, the effect of temperature and strain can be removed from the measurement. This property is indeed a positive feature of this sensor since by benefiting from which, one does not 


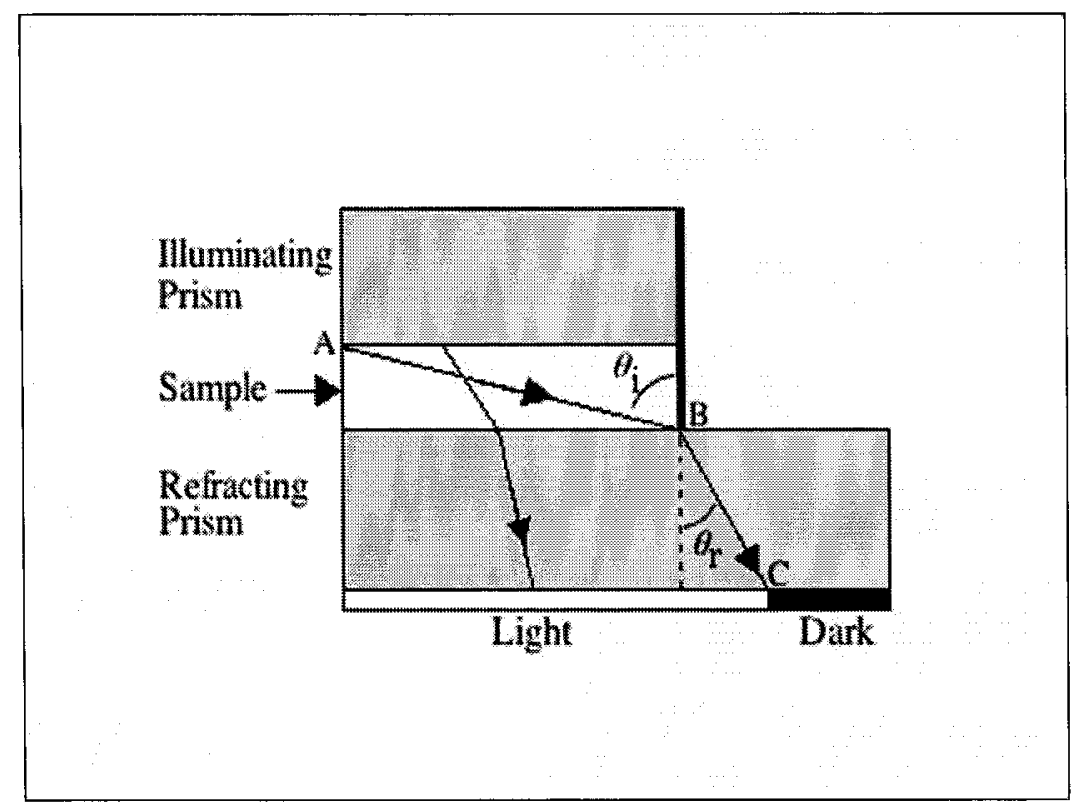

Figure 5.3: Abbe Refractometer, Principles of Operation.

need to use two gratings, one for sensing RI and the other one for sensing temperature.

The refractive index solution used in this work was a water-sugar solution. Sugar solution with different concentrations can provide a wide range of refractive indices from $1.38(30 \%)$ to $1.49(80 \%)$. These refractive indices are all $n_{D}$ values which means the refractive index at the wavelength of $589.3 \mathrm{~nm}$.

In order to measure the refractive index of the sugar solution, a piece of equipment called Abbe Refractometer was used ${ }^{2}$. In the Abbe refractometer, the liquid sample is sandwiched into a thin layer between an illuminating prism and a refracting prism (Figure 5.3). The refracting prism is made of a glass with a high refractive index (e.g., 1.75) and the refractometer is designed to be used with samples having a refractive index smaller than that of the refracting prism. A light source is projected through the illuminating prism, the bottom surface of which is ground (i.e., roughened like a ground-glass joint), so each point on this surface can be thought of as generating light

\footnotetext{
${ }^{2}$ Here I should thank Laura Stuart, from Chemistry Department, Carleton University, who kindly lent me Abbe Refractometer and some more equipment.
} 


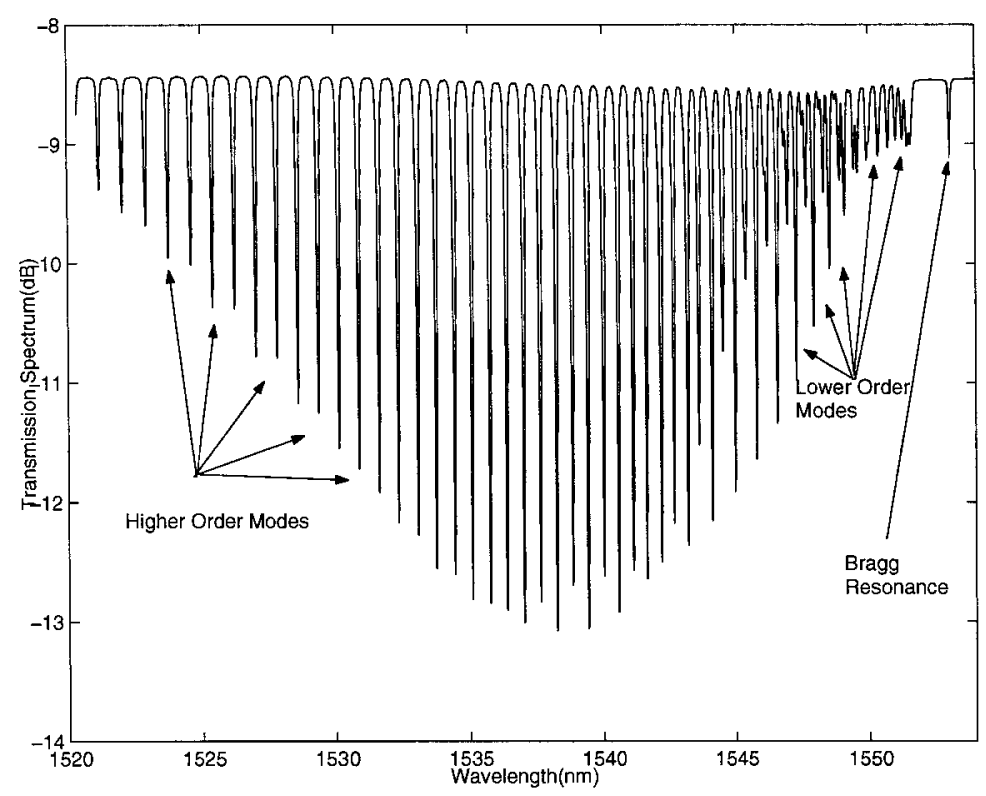

Figure 5.4: A Sample transmission spectrum of TFBG in sugar solution $\left(n_{D}=1.401\right)$.

rays traveling in all directions. Inspection of Figure 5.3 shows that light traveling from point $\mathrm{A}$ to point $\mathrm{B}$ will have the largest angle of incidence $\left(\theta_{i}\right)$ and hence the largest possible angle of refraction $\left(\theta_{r}\right)$ for that sample. All other rays of light entering the refracting prism will have smaller $\theta_{r}$ and hence lie to the left of point C. Thus, a detector placed on the back side of the refracting prism would show a light region to the left and a dark region to the right. Samples with different refractive indexes will produce different angles of refraction and this will be reflected in a change in the position of the borderline between the light and dark regions. By appropriately calibrating the scale, the position of the borderline can be used to determine the refractive index of any sample. The Abbe refractometer is calibrated so that it gives the refractive index at the wavelength of $589.3 \mathrm{~nm}$, the sodium $\mathrm{D}$ line.

The description of the experiment conducted to obtain the transmission spectrum of TFBG was as follow. In each stage, the grating was sandwiched between two glass slides whose space between them contained an amount of sugar solution with a certain 


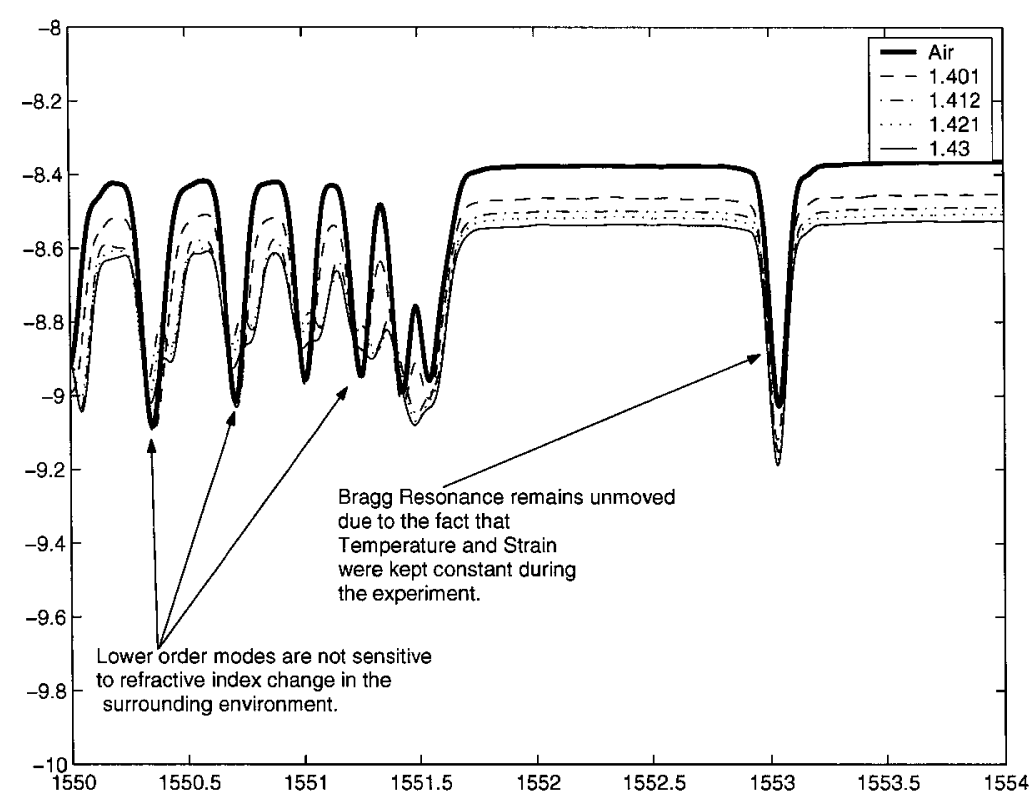

Figure 5.5: Transmission spectrum of TFBG in different SRIs in the vicinity of Bragg resonance.

concentration and thus a specific refractive index. Tunable light was launched into the fiber through the light source of the JDS system and corresponding transmission spectrum was measured and recorded. Figure 5.4 illustrate one of these recorded spectra. This procedure has been repeated for several different sugar solution concentrations (i.e. different refractive indices) and each time the transmission spectrum has been recorded.

Processing the data obtained from experiment has been the subsequent step. In this step, the recorded spectra have been overlaid and if the Bragg resonances did not overlap, they were shifted mathematically to overlap with the Bragg resonance of the spectrum in the air. Transmission spectrum of TFBG in the air has been set the reference in this work, with respect to which the shifts have been calculated. Figure 5.5 illustrates the overlap of the Bragg resonances of different spectra. The thick solid line in the graph refers to the spectrum in the air. As the picture shows, the resonances which occur in the vicinity of Bragg resonance, also referred to as lower 


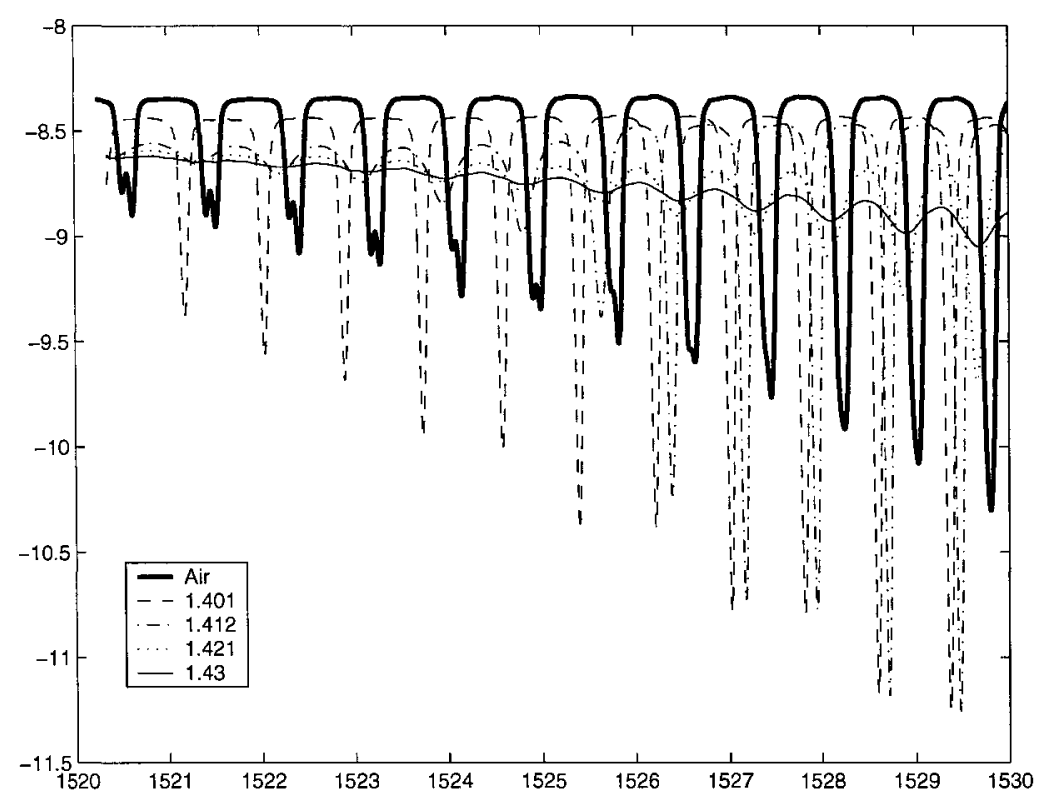

Figure 5.6: Transmission spectrum of TFBG in different SRIs from 1520nm to $1530 \mathrm{~nm}$.

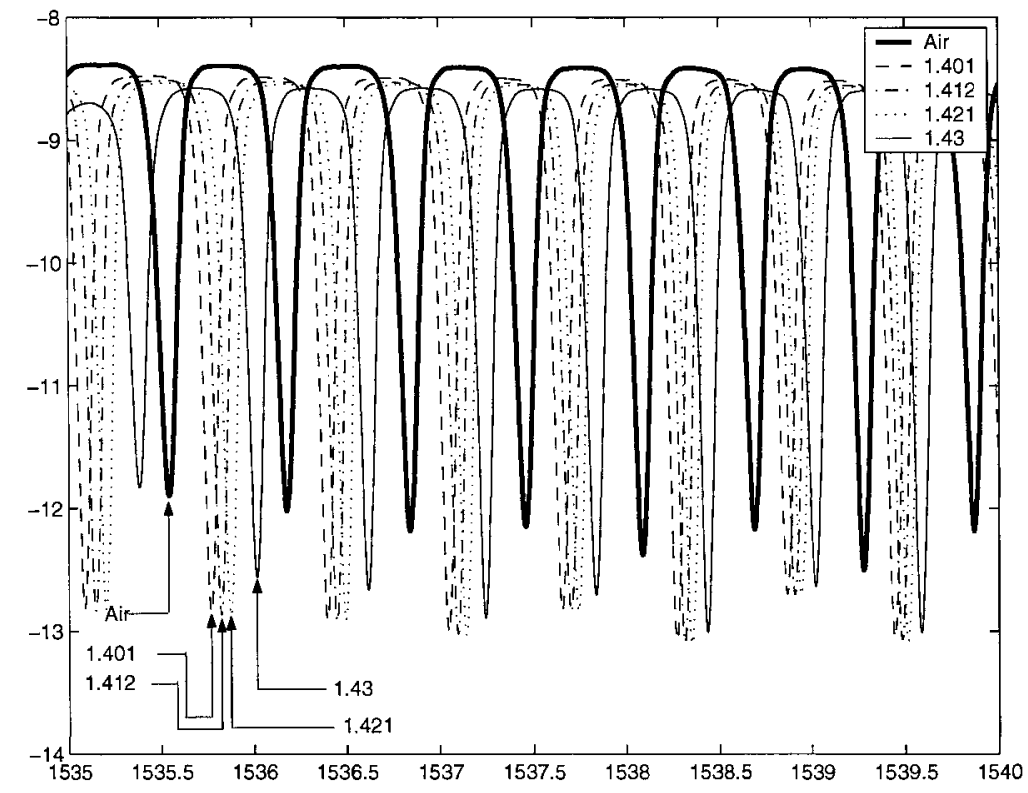

Figure 5.7: Transmission spectrum of TFBG in different SRIs from $1535 \mathrm{~nm}$ to $1540 \mathrm{~nm}$. 
order modes, are not sensitive to external refractive index change. Here it would be useful to repeat this aforementioned point that any change in the position of Bragg resonance is due to change in either the temperature or the strain applied to the sensor and not variation of the external material refractive index. This phenomenon arises due to the fact that Bragg resonance is indeed due to the fundamental core mode resonance (i.e. $\left.L P_{(0,1)}\right)$ which is totally guided along the core and does not sense any change in the surrounding environment but temperature and strain and therefore remains unmoved when the temperature and strain applied on the fiber are constant and external refractive index changes.

Figures 5.6 and 5.7 show the overlaid spectra in more details. Figure 5.6 reveals that as the refractive index of the surrounding material approaches that of the cladding, higher order modes start to disappear. The explanation of this phenomenon is pretty straightforward. As the index contrast between the cladding and outside material decreases, the normalized frequency of the cladding as a waveguide decreases and thus high order cladding modes reach cut off.

The middle wavelength regions such as regions between $1530 \mathrm{~nm}$ and $1540 \mathrm{~nm}$ for the purpose of SRI detection are more suitable than other regions. Figure 5.7 shows a detailed picture of the overlapped spectra in this region. As it is shown on the picture by arrows, as the SRI increases the resonance (the marked mode by arrows is mode $\left.L P_{(1,24)}\right)$ moves to the right (longer wavelengths). By tracking these shifts one can detect the SRI value. In order to track the shifts, first the exact position of the resonance (mode) is determined and then the distance between this resonance and Bragg resonance is calculated. This distance is plotted versus SRI (Figure 5.8). By using this graph and keeping in mind that this graph has been plotted by tracking mode $L P_{(1,24)}$ one can actually determine the refractive index of a surrounding material by determining the amount of shift of the mode and then reading the RI from this graph. Another point to be made here is that in this graph the distance from 


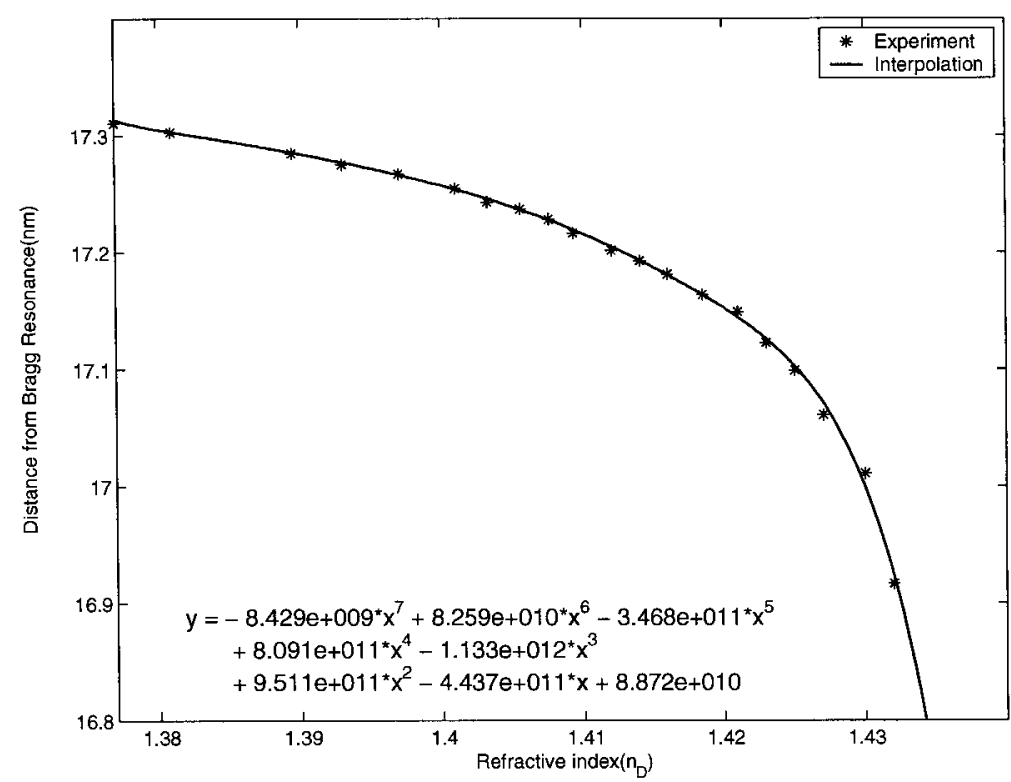

Figure 5.8: Shift in the Mode $L P_{(1,24)}$ due to the SRI change

Bragg resonance is plotted versus $n_{D}$ which is the refractive index at the wavelength 589.3nm. Considering Figure 5.8 as a sensor graph, the first thing that comes to mind is what is the sensitivity of this sensor? That is, for a small change in the SRI value $(\Delta \mathrm{n})$, how much does the resonance displace $\left(\Delta \lambda_{i}\right)$ ?

\subsection{Simulation}

Simulation of the transmission spectrum of a TFBG and getting an exactly matched spectrum with its corresponding experimental one, is a very hard job to my knowledge and I should admit that in this project there has not been enough work done on this part.

The software used to simulate TFBG is Optigrating, from Optiwave. The problem is that this software does not take into account the dispersion of the core and cladding material and because the simulation should be performed over a wide range 


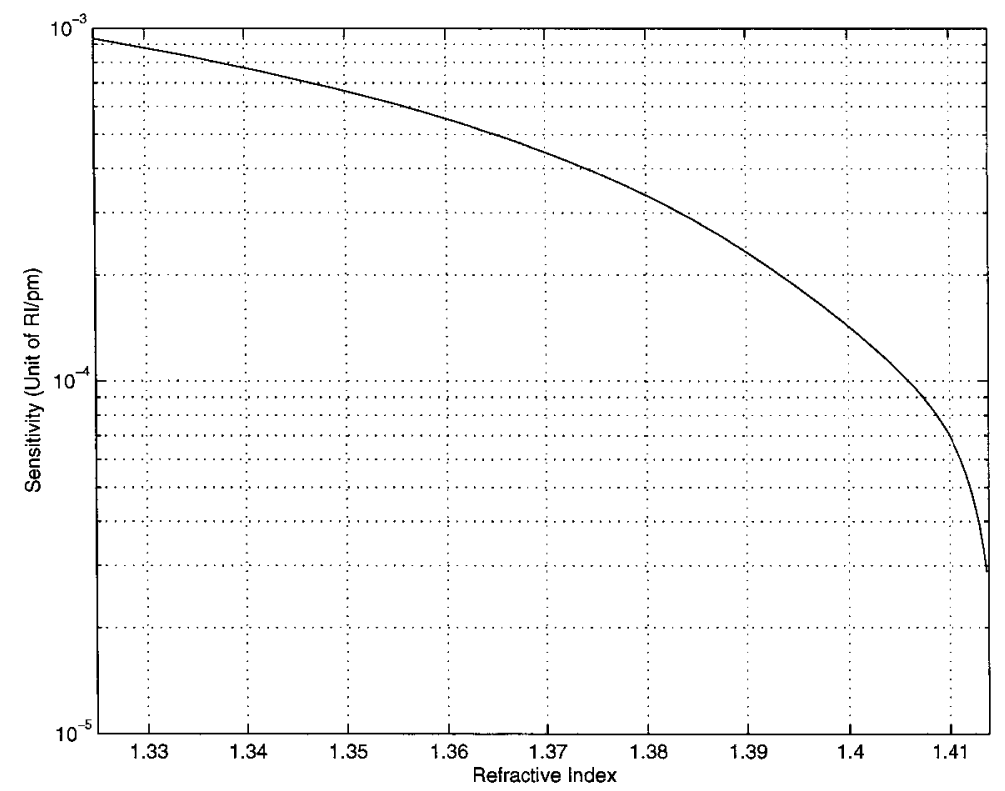

Figure 5.9: Sensitivity of Mode $L P_{(1,24)}$, (Unit of $\left.R I / p m\right)$.

of wavelengths (at least 35 or $40 \mathrm{~nm}$ ), and because the values of the core and cladding effective refractive indices should be calculated precisely in order to get the location of resonances correct, this foible of the software cause significant errors and thus yields the location of the resonances totally off.

To overcome this drawback, my colleague, Ch. Chen, proposed a method to perform the simulation. In his method the simulation is done in several steps. In each step instead of simulating the grating over a whole range of wavelength, it is only performed over a small one, let's say 4 or 5 nanometers and then in this range the exact values of core and cladding refractive indices (by taking into account the dispersion of the core and the cladding) are employed. He was able to obtain a very good match between the simulated spectrum and the experimental one by applying this method.

Finding exact values of refractive indices of core and cladding at the very beginning is also another impediment in the way of performing the simulation. First of all, the 


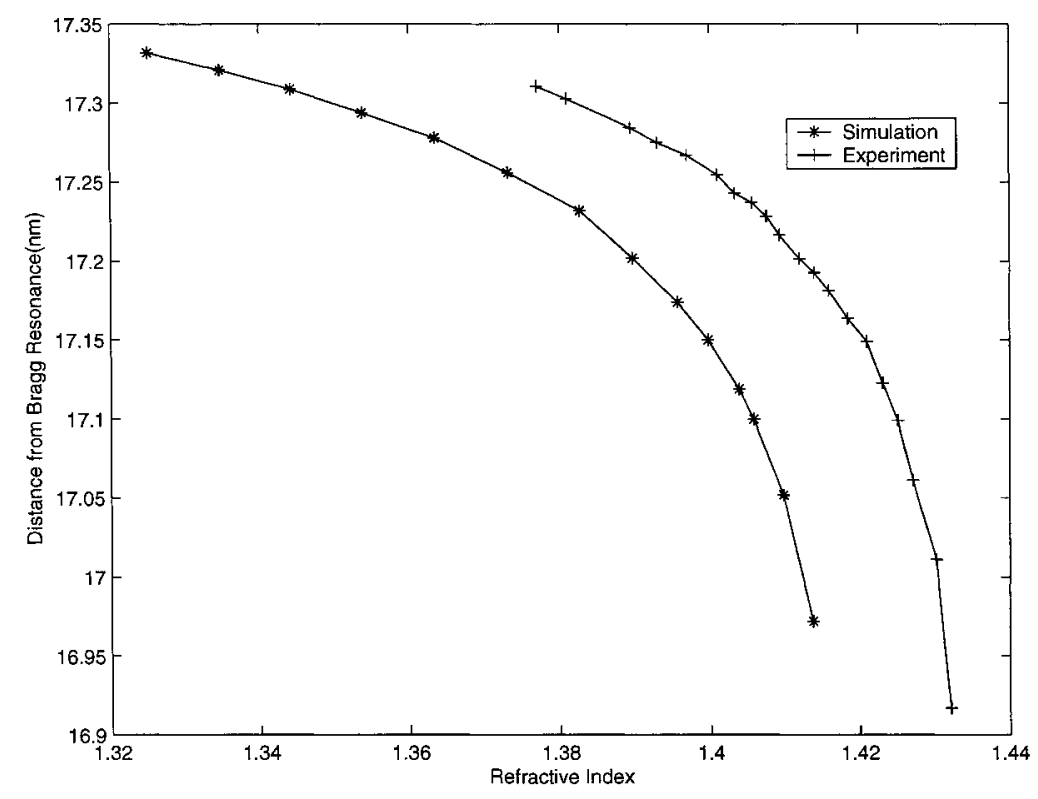

Figure 5.10: Mode $L P_{(1,24)}$ shift due to SRI change, simulation and experiment.

exact values of index of refraction of the core and cladding for a single mode fiber is not provided by the manufacturer and people use a variety of numbers in the literature. Secondly, even if the exact value of the refractive index was known, it was not useful in the simulation because writing the grating on the fiber would change the refractive index (depending on UV radiation time, power of the laser used to inscribe the grating on the fiber and so on and so forth) of the fiber and thus the index of refraction of the processed fiber is different from the unprocessed one. The amount of change is not known easily to us unless one measures it. Starting with two arbitrary values close to what thought to be the exact values and playing with them to get the distance between the Bragg resonance and the ghost mode resonance (the first resonance before the Bragg peak) is the way that my colleague, Chen, employed to overcome this difficulty.

Apart from what was explained above on the hardship of TFBG simulation, a very simple simulation was performed to verify the experimental results. The simulation 
was only performed in the vicinity of wavelength $1535 \mathrm{~nm}$, where the mode $L P_{(1,24)}$ resonates. Figure 5.10 depicts the result of this simulation. The curves are not overlapping and the reason for this is that the experimental graph is in fact plotted "Distance from Bragg resonance of the mode $L P_{(1,24)}$ " versus " $n_{D}$ " values while the simulation graph is plotted "Distance from Bragg resonance of the mode $L P_{(1,24)}$ " versus " $n_{1535}$ " (refractive index at $1535 \mathrm{~nm}$ ). In order to conduct the experiment, as it was mentioned above, sugar-water solution with different concentrations, was used to provide several needed refractive indices. The only equipment we had available to measure the refractive index of the solution was Abbe Refractometer which yields the index of refraction at the wavelength of $589.3 \mathrm{~nm}$. In order to perform the simulation in the vicinity of $1535 \mathrm{~nm}$, it was necessary to input into the software the values of the refractive index of surrounding material in this wavelength. The task was now to find a way to map the refractive index of the sugar-water solution at the wavelength of $589.3 \mathrm{~nm}$ to the wavelength of $1535 \mathrm{~nm}$. The simplest way to do this mapping is to suppose that there is linear relationship between $n_{D}$ and $n_{1535}$ for different concentrations. ${ }^{3}$

Figure 5.11 illustrates the result of using a linear mapping function to transform the $n_{D}$ values of sugar-water solution to $n_{1535}$ values (The mapping function is: $n_{1535}=1.307 \times n_{D^{-}}$0.457). Using more complicated mapping functions may yield even better matching between simulation and experimental curves.

The last issue that was looked at is whether the results depend strongly on the

\footnotetext{
${ }^{3}$ In a paper titled Empirical Equation for the Index of Refraction of Seawater, published by X. Quan and E. S. Fry, Applied Optics, Vol 34, No 18, June 1995; an empirical equation for the index of refraction of water as a function of temperature, salinity and wavelength was derived. In equation 3 of this paper, it is shown that RI is a linear function of salinity (S), at a wavelength $\lambda$ and temperature $\mathrm{T}$, for example, $n_{D}=\mathrm{A} . \mathrm{S}+\mathrm{B}$ and $n_{1535}=\mathrm{C} . \mathrm{S}+\mathrm{D}$; where $\mathrm{S}$ is salinity in $\%$ and $\mathrm{A}, \mathrm{B}, \mathrm{C}$, and $\mathrm{D}$ are all constants. By combining two equations and eliminating $\mathrm{S}$ factor in them one obtains this equation: $n_{1535}=\mathrm{C} .\left(\left(n_{D}-\mathrm{B}\right) / \mathrm{A}\right)+\mathrm{D}=\mathrm{E} \cdot n_{D}+\mathrm{F}$, again here $\mathrm{E}$ and $\mathrm{F}$ are constants provided that temperature is kept fixed. This inspires us that the same relationship also holds for sugar-water solution.
} 


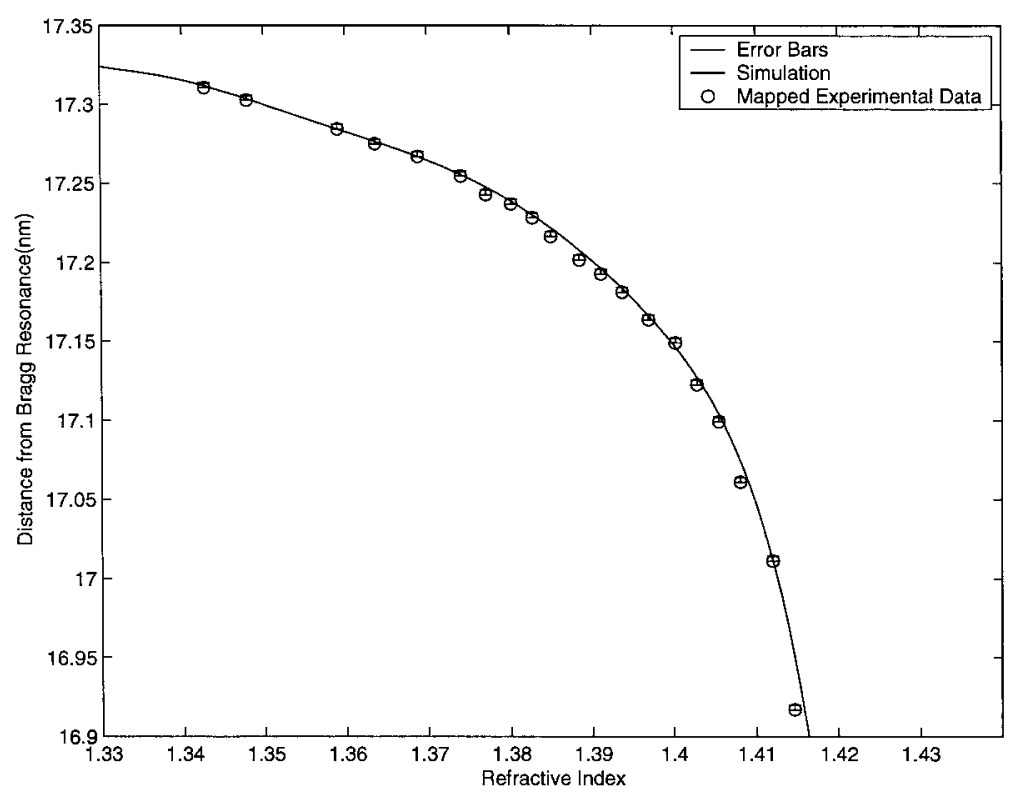

Figure 5.11: Overlap of simulation and experimental curve using a linear mapping function to map RI of sugar-water solution from $589.3 \mathrm{~nm}$ to $1535 \mathrm{~nm}$. Error bars are also shown on the graph. The error in reading the $R I$ value from Abbe Refractometer is $10^{-4}$ and the error in reading the wavelength is $\pm 3 p m$.

transverse mode shape or only on the wavelength distance between the Bragg resonance and the cladding mode resonance used. This is important because it is often difficult (or even impossible) to unequivocally identify the fiber mode associated with a particular resonance. For example, detailed investigation of the TFBG spectrum in the vicinity of wavelength $1535 \mathrm{~nm}$ revealed that in the place where mode $L P_{(1,24)}$ resonances there is also another mode, $L P_{(3,23)}$. Figure 5.12 shows the shift in the location of these two modes. As inferred from the picture, although the mode shapes of these two modes are completely different from each other, because they are sitting in the same distance from Bragg resonance, they follow the same shift pattern. This is a very important and noticeable property of TFBG that we do not have to worry about the identity of the mode to be used for measuring the SRI but only about its distance from the Bragg resonance. 


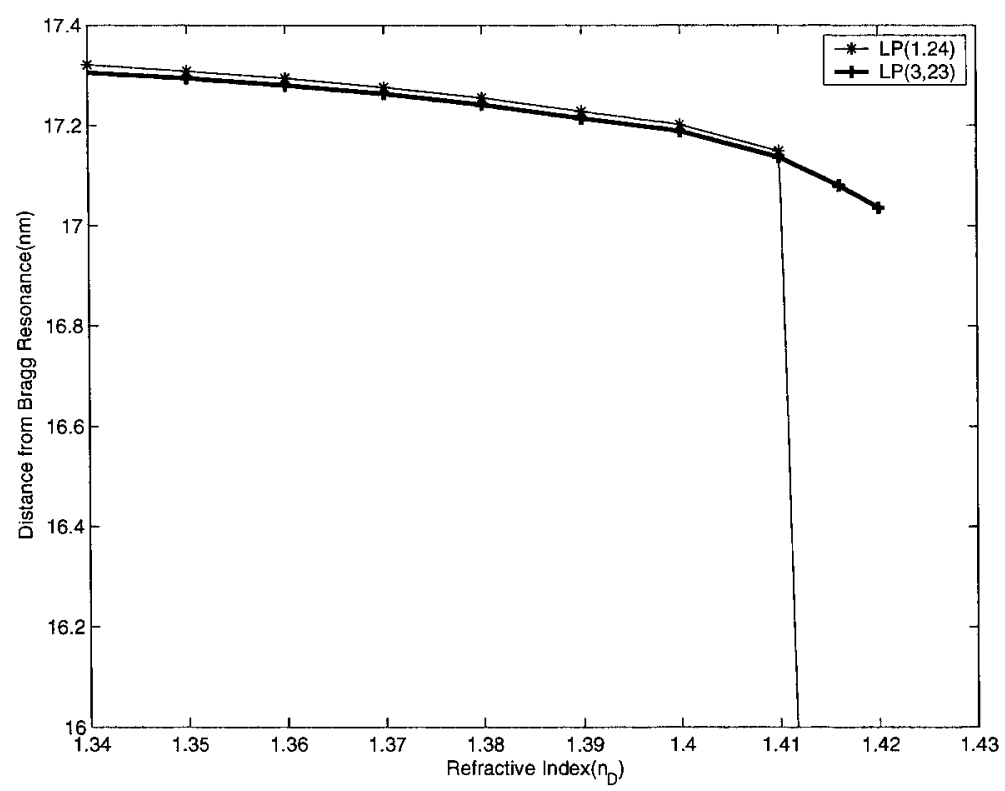

Figure 5.12: Shift in the location of modes $L P_{(1,24)}$ and $L P_{(3,23)}$ due to SRI change.

\subsection{Interrogation of the TFBG with AWG}

The objective of this section is to present the preliminary results of employing an AWG as the interrogator unit for a TFBG. A typical TFBG spectrum was taken and was introduced as the input of the AWG. Then the power was integrated over the output channels of AWG. The AWG output response used here was not a real one but a very simplified mathematical one (a train of $\operatorname{rect}(.)^{4}$ functions with an arbitrary width and height 1 was employed instead of a real AWG output response). Figure 5.13 shows the output of the AWG with three different inputs. In these graphs channel number one has been fixed on the Bragg resonance in all the three different cases. As mentioned earlier, the change of refractive index of the surrounding environment does not affect lower order modes (i.e. modes in the vicinity of the Bragg resonance). This fact is clearly shown in Figure 5.13. The channels in the vicinity of channel

\footnotetext{
${ }^{4} \mathrm{~A}$ rect function is defined as: $\Pi(x)=u\left(x+\frac{1}{2}\right)+u\left(x-\frac{1}{2}\right)$ where $\mathrm{u}(\mathrm{x})$ is called the step function and is defined zero for negative arguments and one for positive arguments.
} 

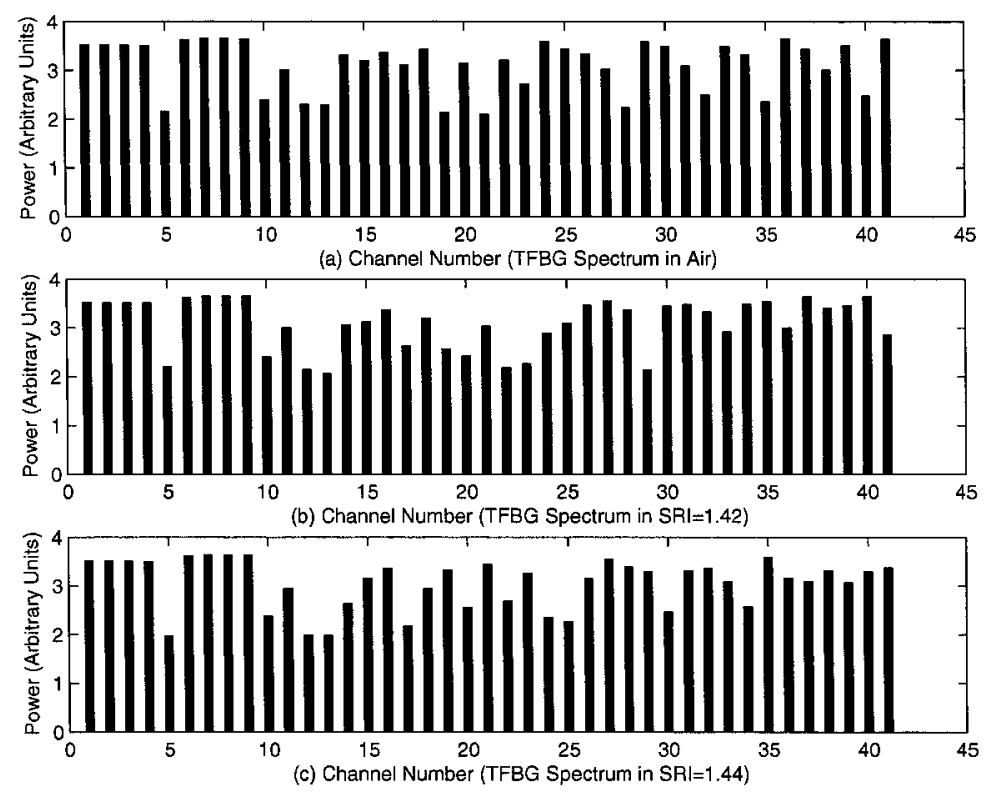

Figure 5.13: Power integrated over the output channel of an $A W G$ with the TFBG spectrum as the input. (a): Output of the AWG with the TFBG Spectrum in Air. (b): Output of the $A W G$ with the TFBG Spectrum in $S R I=1.42\left(n_{D}=1.42\right)$. (c): Output of the $A W G$ with the TFBG Spectrum in $S R I=1.44\left(n_{D}=1.44\right)$.

1, show the same output for three different cases. To show this fact more clearly one should take a look at Figure 5.14 where the AWG output response with TFBG spectrum in the air is taken as the reference and AWG output responses with TFBG spectra's in SRI=1.42 and 1.44 subtracted from it respectively. AS seen in the graph, the difference in the vicinity of the channel number 1 is zero or very close to zero which means the change in the SRI does not affect the amount of power in the lower order modes while going from lower order modes to the higher order modes, shows a more drastic difference.

This kind of graph, provides us with very useful information. For example, by introducing threshold lines (discretization levels) in these graphs (see Figure 5.14) one can convert the information of the graphs into digital format which ultimately 

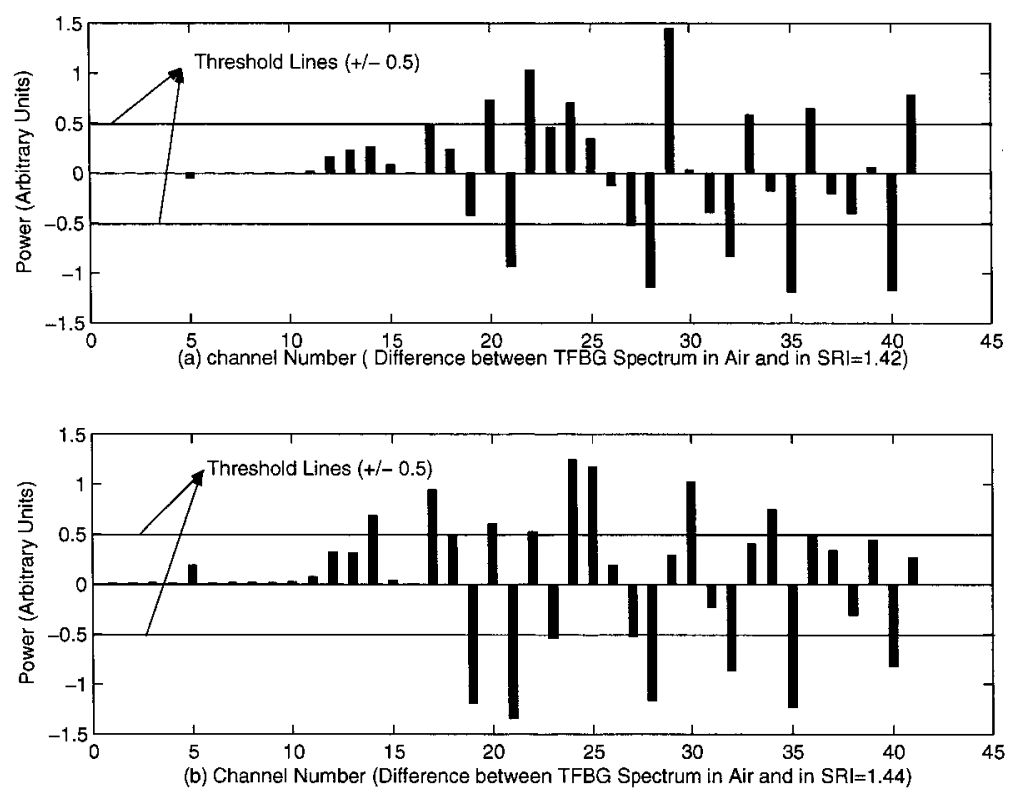

Figure 5.14: Difference between the output responses of the $A W G$ with the output response of Air as the reference and, (a) : the output response of the $S R I=1.42$ subtracted from it; (b) : the output response of the SRI=1.44 subtracted from it.

enables one to employ $\operatorname{DSP}^{5}$ techniques to handle the data. Here as an example we have introduced threshold lines at \pm 0.5 , which means every bar with a value below -0.5 is associated with -1 , between -0.5 and +0.5 is associated with 0 and larger than +.05 is associated with +1 (Figure 5.15) . By doing this, the AWG output response in each case (or part of the response, i.e. a few modes only to be taken in to account) can be introduced with a code word. For instance, as it is shown in Figure 5.15, the AWG response of channels 17 to 25 can be represented by a code word of $(0,0,0,1,-$ $1,1,0,1,0)$ in the case of SRI $=1.42$ and by a code word of $(1,0,-1,1,-1,1,-1,1,1)$ in the case of SRI=1.44. Now employing powerful available DSP techniques enables us to handle these code words. The point that these results are absolutely preliminary and need more elaboration should be pointed out here again. This small piece of work was done only to give an overall idea on one of the possible ways of employing an

\footnotetext{
${ }^{5}$ Digital signal Processing
} 

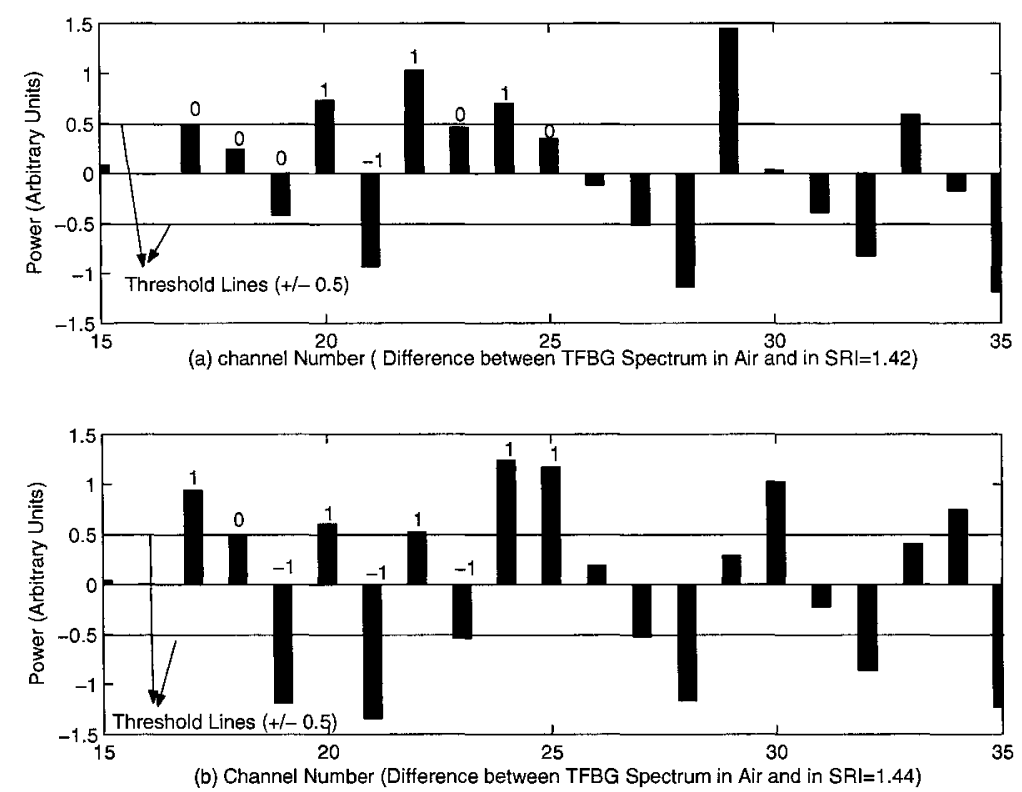

Figure 5.15: Introducing discretization levels and converting the data to digital format.

AWG as the TFBG sensor interrogator.

\subsection{Conclusion}

The TFBG as a special kind of grating which has the capability of sensing refractive index in addition to temperature and strain was investigated. It was shown that different resonances in the spectrum of TFBG displace due to change in the surrounding material refractive index and by tracking these shifts one can actually realize a refractive index sensor. The sensitivity of this sensor was studied and it was shown that in some regions the sensor responds linearly to SRI change while in other places the response of the sensor (shift in the resonance location) increases up to a point where the resonance disappears (the mode is no longer guided along the cladding and starts to radiate). These results were verified by numerical simulation. The problem of unavailability of RI of the sugar in the wavelength of $1535 \mathrm{~nm}$ was solved by using 
a linear mapping function to map the values of $n_{D}$ on the the values of $n_{1535}$. At the end it was pointed out that the shape of the mode does not affect the shift pattern of the resonance as long as the resonance is sitting a certain distance away from Bragg resonance.

The last section of this chapter, dealt with a very simple method to interrogate the TFBG spectrum with an AWG. Although the employed AWG was not a real one, interesting preliminary results were achieved. 
"A conclusion is the place where you got tired of thinking."

- Martin H. Fischer

\section{Chapter 6}

\section{Concluding Remarks}

In this chapter, we briefly review the subjects of this thesis, and suggest some directions for future research work.

\subsection{Summary of Thesis Research}

As a part of this multidisciplinary work, the Arrayed Waveguide Grating as a promising choice to be used in sensor interrogating applications was studied. We saw that properties such as low cost, compactness of size, ease of fabrication and high accuracy in measuring both shifts of the signal along wavelength axis and variations of the signal amplitude, are the motivating factor to consider AWG as an ideal interrogator unit. Operating principles of this device, based on which the light propagates in the first free space coupler, gets collected by arrayed waveguides at end of this region, and converges to one of the output waveguides, were explained.

From a system point of view, we cast an eye upon AWG as a device designed to exhibit specifications such as certain amount of bandwidth, specific channel spacing, sufficiently low crosstalk level and least possible amount of loss. In order to realize these expectations, our means were the geometrical parameters of the device including waveguide spacings, length of free space couplers and the length difference between the waveguides in the array. The step by step design procedure proposed in this work, 
was the method based on which we used equations relating geometrical parameters to system specifications, to realize a practically useful device satisfying our requirements. Finally, two interrogation schemes were set forth and discussed.

Second part of this thesis dealt with TFBG as a refractive index sensor. Obliqueness of the grating planes in TFBG, was the feature that led to the additional property of capability of sensing the RI of the surrounding environment over the common properties of a FBG (which was only capable of sensing temperature and strain). We observed that because of this obliqueness, the light, in encounter with grating planes, was partly scattered to cladding and thus was carried by cladding modes. We saw that the propagation constants of these modes were highly sensitive to the variations of the external refractive index. Any change in SRI made the cladding mode resonances to displace. The idea of tracking these displacements in order to detect the changes in SRI value, was examined with experiment and was verified by simulation. The sensitivity of the sensor, by which term we mean the amount of displacement the resonance undergoes due to a differential change in RI, was another issue studied in this work. It was noticed that in addition to the fact that different resonances have different sensitivities, each resonance over different RI ranges also exhibits different sensitivities. In smaller values of RI, the resonance responses linearly (the curve of "Distance of the Resonance from Bragg resonance" versus "Refractive Index" was approximately linear) to the change in RI whereas after passing a threshold value, the resonance experiences very drastic shifts and ultimately disappears. The reason of this disappearance is that as the SRI value approaches the RI of the cladding, the mode is not anymore a guided one along cladding-external environment surface but a propagating one. 


\subsection{Possible Future Work}

In the continuation of this work, for short-term research, an interesting problem to look into, in the context of AWG based sensor interrogator, is fabrication and characterization of a properly designed AWG. The proposed design in this work uses an output waveguide separation of $50 \mu \mathrm{m}$ at the edge of the wafer. This choice of spacing, besides shrinking the overall size of the device to be able to fit on a smaller wafer (for fabrication), makes the device to able to employed along with a detector array. Studying the feasibility of coupling AWG either through output waveguides to a detector array or directly at the end of second free space coupler, is another interesting subject.

In this work, the operating central wavelength of AWG has been around $1550 \mathrm{~nm}$, while it is also interesting to design, fabricate and characterize AWGs with operating wavelength regions at shorter wavelengths $(<1 \mu \mathrm{m})$ for compatibility with low cost light sensors and detectors.

For long-term research, we suggest investigating the interrogating schemes of TFBG using AWG. Inasmuch as interrogating schemes discussed in this thesis (taken from literature) are mostly for interrogating a single FBG or at most a multiple number of them and not specifically suitable for TFBG, it would be very interesting if one can come up with a proper scheme specifically developed for TFBG. A very small piece of work was done in this thesis but it is absolutely insufficient.

In relation with TFBG, numerical simulation of the spectrum of TFBG is a very challenging issue. Besides this, from a sensor point of view, fully characterization of the TFBG as a RI sensor sounds necessary. Preliminary results show that TFBGs exhibit a high level of PDL which may be problematic in some applications and could be a subject of further studies in this context. 


\section{Appendix A}

\section{AWG Based Interrogator Design Paramaters}

In this appendix, a full description of the AWG design 3, mentioned in Chapter 4, is given. Besides optical and geometrical parameters, system specifications are also stated. This design is indeed customized to be employed in interrogating a TFBG with a tilt angle of 6 degrees. The channel spacing of the AWG dictated by the average spacing between TFBG resonances. Inasmuch as the spacing between resonances is not the same all over the spectrum, the ideal design would be the one with nonuniform channel spacing, but using a non-uniform design, would be a complicated one and it is a main concern to keep the design as simple as possible in order to lower the cost. The simulation results of this design, specially its transmission spectrum was given in chapter 4 . Tables A.1, A.2 and A.3 yield the full description of the design. 


\begin{tabular}{|l|c|c|}
\hline Optical Parameters & Value & Unit \\
\hline Waveguide core hight & 5 & $\mu \mathrm{m}$ \\
\hline Waveguide core width & 5 & $\mu \mathrm{m}$ \\
\hline Lower cladding thickness & 5 & $\mu \mathrm{m}$ \\
\hline Top cladding thickness & 5 & $\mu \mathrm{m}$ \\
\hline Waveguide core index & 1.4558408 & \\
\hline Lower cladding refractive index & 1.444 & \\
\hline Top cladding refractive index & 1.444 & \\
\hline$N_{\text {eff } \text { of the Slab }}$ & 1.45584087 & \\
\hline$N_{\text {eff } \text { of the channel }}$ & 1.4543699 & \\
\hline
\end{tabular}

Table A.1: Optical Parameters of Design 3

\begin{tabular}{|l|c|c|}
\hline Mask Parameters & Value & Unit \\
\hline Number of arrays & 181 & \\
\hline Length of the slab & 11151.12 & $\mu \mathrm{m}$ \\
\hline Taper width & 7 & $\mu \mathrm{m}$ \\
\hline Taper length & 100 & $\mu \mathrm{m}$ \\
\hline Taper type & Linear & \\
\hline Extension after taper & 0.0 & $\mu \mathrm{m}$ \\
\hline Channel effective index for taper & 1.4543699 & \\
\hline Array separation & 9.0 & $\mu \mathrm{m}$ \\
\hline Channel separation & 25 & $\mu \mathrm{m}$ \\
\hline Number of output waveguides & 65 & \\
\hline Slab's angle with horizontal line & 91.2454 & Degree \\
\hline Distance between two output ports & 50 & $\mu \mathrm{m}$ \\
\hline
\end{tabular}

Table A.2: Mask Parameters of Design 3 


\begin{tabular}{|l|c|c|}
\hline specifications & Value & Unit \\
\hline Number of channels & 65 & \\
\hline Central wavelength & 1539 & $\mathrm{~nm}$ \\
\hline Channel spacing & 0.5 & $\mathrm{~nm}$ \\
\hline Free spectral range & 32.5 & $\mathrm{~nm}$ \\
\hline Passband @ 3 dB & 0.22 & $\mathrm{~nm}$ \\
\hline Crosstalk (adjacent) & -24 & $\mathrm{~dB}$ \\
\hline Crosstalk (non-adjacent) & -35 & $\mathrm{~dB}$ \\
\hline Device Size & $23 \times 19$ & $\mathrm{~mm}$ \\
\hline Length increment & 49.7349402 & $\mu \mathrm{m}$ \\
\hline Grating Order & 47 & \\
\hline
\end{tabular}

Table A.3: System Specifications of Design 3 


\section{Bibliography}

[1] M. Smit and C. Dam, "PHASAR-Based WDM-Devices: Principles, Design and Applications," IEEE J. Select. Topics Quantum Electron., vol. 2, pp. 236-250, June 1996.

[2] D. Norman, D. Webb, and R. Pechstedt, "Interrogation of fiber Bragg grating using an Arrayed Waveguide Grating," Measurement science and technology, vol. 16, pp. 691-698, March 2005.

[3] G. Xiao, P. Zhao, F. Sun, Z. Lu, Z. Zhang, and C. Grover, "Interrograting fiber Bragg grating sensors by thermally scanning a demultiplexer based on Arrayed Waveguide Grating," Opt. Lett., vol. 29, pp. 2222-2224, October 2004.

[4] S. Mell, K. Liu, and R. Measures, "Practical fiber-optic Bragg grating strain gauge system," Appl. Opt., no. 32, pp. 3601-3609, 1993.

[5] A. Kersey, T. Berkoff, and W. Morey, "Multiplexed fiber Bragg grating strainsensor system with a fiber Fabry-Perot wavelength filter," Opt. Lett., vol. 18, pp. 1370-1372, August 1993.

[6] M. Davis and A. Kersey, "All-fiber Bragg gratin strain-sensor demodulation technique using a wavelength division coupler," Electron. Lett., vol. 30, pp. 75-77, January 1994.

[7] M. Xu, H. Geiger, J. Archambault, L. Reekie, and J. Dakin, "Novel interrogatin system for fiber Bragg grating sensors using an Acousto-Optic tunable filter," Electron. Lett., vol. 29, pp. 1510-1511, August 1993. 
[8] A. Kersey, T. Berkoff, and W. Morey in Proceedings of first european conference on smart structures and materials, (Bellingham), pp. 61-67, 1992.

[9] C. Nadler, Polarization insensitive Arrayed Waveguide Grating wavelength multiplexeres for optical communications. $\mathrm{PhD}$ thesis, Eidgenossische Techn. Hochsch., Zurich,Switzerland, 1999.

[10] K. Okamoto, Fundamentals of Optical Waveguides. San Diego: Academic Press, 2000.

[11] T. Mizuno, T. Kitoh, M. Ishii, Y. Inoue, T. Saida, M. Itoh, T. Shibata, and Y. Hibino, "Compact and Low-Loss Arrayed Waveguide Grating module with tolerance-relaxed spot-size convertor," IEEE Photon. Technol. Lett., vol. 15, pp. 239-241, February 2003.

[12] S. Day, J. Stagg, D. Moule, S. Clemens, C. Rogers, S. Ohija, T. Clapp, J. Brook, and J. Morley, "The ellimination of side lobes in the Arrayed Waveguide WDM," Integrated Photonics Research, pp. 48-51, 1996.

[13] C. Lee, W. Chen, Q. Wang, Y. Chen, W. Bernard, D. Stone, R. Smith, R. Mincher, and I. Stewart, "The role of photomask resolution on the performance of Arrayed-Waveguide Grating devices," J. Lightwave Technol., vol. 19, pp. 1726-1733, November 2001.

[14] Y. Chu, X. Zheng, H. Zhang, X. Liu, and Y. Cuo, "The impact of phase errors on Arrayed Waveguide Gratings," IEEE J. Select. Topics Quantum Electron., vol. 8, pp. 1122-1129, November/December 2002.

[15] M. Oguma, T. Kitoh, K. Jinguji, T. Shibata, A. Himeno, and Y. Hibino, "Passband-Width broadening design for WDM filter lattice-form interleave filter and Arrayed Waveguide Gratings," IEEE Photon. Technol. Lett., vol. 14, pp. 328-330, March 2002.

[16] H. Yamada, K. Takada, Y. Inoue, Y. Hibino, and M. Horiguchi, "10GHz-spaced Arrayed-Waveguide Grating multiplexer with phase error compensation thin-film heaters," Electron. Lett., vol. 31, pp. 360-361, March 1995. 
[17] K. Okamoto and A. Sugita, "Flat spectral response Arrayed-Waveguide Grating multiplexer with parabolic waveguide horns," Electron. Lett., vol. 32, pp. 16611662, August 1996.

[18] T. Kamalaski, T. Sphicopoulos, and D. Syviridis, "An estimation of performance degradation due to fabrication errors in AWGs," J. Lightwave Technol., vol. 20, pp. 1779-1787, September 2002.

[19] Y. Sano and T. Yoshino, "Fast optical wavelength interrogator employing Arrayed Waveguide Wrating for distributed fiber Bragg grating sensor," IEEE Photon. Technol. Lett., vol. 21, pp. 132-139, January 2003.

[20] A. Othonos and K. Kalli, Fiber Bragg Grating, fundamentals and applications in telecommunications and sensing. House Publishers, Boston,: Artech House Publisher, 1999.

[21] G. P. Agrawal, Lightwave Technology, Components and Devices. Wiley Interscience, John Wiley \& Sons, Inc., 2004.

[22] T. Erdogan, "Fiber Grating Spectra," J. Lightwave Technol., vol. 15, pp. 12771294, August 1997.

[23] A. Kersey, M. Davis, H. Patrik, M. LeBlanc, K. Koo, C. G. Askins, M. Putnam, and E. Friebele, "Fiber Grating Sensors," J. Lightwave Technol., vol. 15, pp. 1442-1463, August 1997.

[24] X. Shu, Y. Lin, D. Zhao, B. Gwandu, F. Floreani, L. Zhang, , and I. Bennion, "Dependence of temperature and strain coefficients on fiber grating type and its application to simultaneous temperature and strain measurement," Opt. Lett., vol. 27 , no. 8, pp. 701-703, 2002.

[25] X. Chen, K. Zhou, L. Zhang, and I. Bennion, "Simultaneous measurement of temperature and external refractive index by use of a hybrid grating in D fiber with enhanced sensitivity by HF etching," Appl. Opt., vol. 44, pp. 178-182, 2005. 
[26] C. Caucheteur and P. Megret, "Demodulation technique for weakly tilted fiber Bragg grating refractometer," IEEE Photon. Technol. Lett., vol. 17, pp. 27032705, December 2005. 